\title{
Highest weight modules and polarized embeddings of shadow spaces
}

\author{
Rieuwert J. Blok
}

Received: 14 October 2009 / Accepted: 26 October 2010 / Published online: 18 November 2010 (C) Springer Science+Business Media, LLC 2010

\begin{abstract}
The present paper was inspired by the work on polarized embeddings by Cardinali et al. (J. Algebr. Comb. 25(1):7-23, 2007) although some of our results in it date back to 1999. They study polarized embeddings of certain dual polar spaces, and identify the minimal polarized embeddings for several such geometries. We extend some of their results to arbitrary shadow spaces of spherical buildings, and make a connection to work of Burgoyne, Wong, Verma, and Humphreys on highest weight representations for Chevalley groups.

Let $\Delta$ be a spherical Moufang building with diagram $\mathrm{M}$ over some index set $I$, whose strongly transitive automorphism group is a Chevalley group $G(\mathbb{F})$ over the field $\mathbb{F}$. For any non-empty set $K \subset I$ let $\Gamma$ be the $K$-shadow space of $\Delta$. Extending the notion in to this situation, we say that an embedding of $\Gamma$ is polarized if it induces all singular hyperplanes. Here a singular hyperplane is the collection of points of $\Gamma$ not opposite to a point of the dual geometry $\Gamma^{*}$, which is the shadow geometry of type $\operatorname{opp}_{I}(K)$ opposite to $K$. We prove a number of results on polarized embeddings, among others the existence of (relatively) minimal polarized embeddings.

We assume that $G(\mathbb{F})$ is untwisted. In that case, the point-line geometry $\Gamma$ has an embedding $e_{K}$ into the Weyl module $V\left(\lambda_{K}\right)_{\mathbb{F}}^{0}$ of highest weight $\lambda_{K}=\sum_{k \in K} \lambda_{k}$. We show that this embedding is polarized in the sense described above. We then prove that the minimal polarized embedding relative to $e_{K}$ exists and equals the unique irreducible $G(\mathbb{F})$-module $L\left(\lambda_{K}\right)$ of highest weight $\lambda_{K}$. More precisely we show that the polar radical of $e_{K}$ (the intersection of all singular hyperplanes) coincides with
\end{abstract}

Dedicated to Karin, Anna, and Sophie.

This research was completed in part while visiting the University of Siena on a grant from the Gruppo Nazionale per le Strutture Algebriche, Geometriche e le loro Applicazioni in the summers of 2007 and 2008.

R.J. Blok $(\bowtie)$

Department of Mathematics and Statistics, Bowling Green State University, Bowling Green, OH 43403, USA

e-mail: blokr@member.ams.org 
the radical of the contravariant bilinear form considered by Wong to obtain the irreducible (restricted) representations of $G(\mathbb{F})$ in positive characteristic.

This viewpoint allows us to "recognize" the irreducible $G(\mathbb{F})$-modules of highest weight $\lambda_{K}$ geometrically as minimal polarized embeddings of the appropriate shadow space.

Keywords Building · Shadow space · Grassmannian · Polarized embedding · Chevalley group $\cdot$ Highest weight module $\cdot$ Representation theory

\section{Introduction and preliminaries}

\subsection{Basic definitions}

Vector spaces, group actions and modules Throughout this paper $\mathbb{F}$ shall denote a field. By a field we shall mean a commutative division ring. Unless otherwise specified, vector spaces will be left vector spaces. Actions of groups, or algebras on a vector space shall therefore also be left actions, unless otherwise specified.

Point-line geometries, hyperplanes and embeddings We assume the reader is familiar with the concept of a point-line geometry $\Gamma=(\mathcal{P}, \mathcal{L})$ (also called a partial linear rank two incidence geometry), see e.g. [10, 33]. In a partial linear space we can and shall often identify each line with the set of points incident to it. By a subspace of $\Gamma$ we mean a subset $S \subseteq \mathcal{P}$ such that if $l \in \mathcal{L}$ and $l \cap S$ contains at least two points, then $l \subset S$. Clearly the intersection of subspaces is a subspace and consequently it is natural to define the subspace generated by a subset $X$ of $\mathcal{P},\langle X\rangle_{\Gamma}$, to be the intersection of all subspaces of $\Gamma$ that contain $X$. A hyperplane of $\Gamma$ is a proper subspace meeting every line. A set of points is called connected if its collinearity graph is connected. We recall the following.

Lemma 1.1 If $H$ is a hyperplane such that $\Gamma-H$ is connected, then $H$ is a maximal subspace of $\Gamma$.

The projective point-line geometry of a vector space $V$ is the point-line geometry $\mathbb{P}(V)=(\mathcal{P}(V), \mathcal{L}(V))$ whose points and lines are the 1 -spaces and 2-spaces of $V$ with incidence given by symmetrized inclusion.

A full projective embedding (or simply embedding) of $\Gamma$ is a pair $(e, V)$, where $V$ is a vector space and $e: \mathcal{P} \hookrightarrow \mathcal{P}(V)$ is an injective map such that

(E1) $\langle e(\mathcal{P})\rangle_{V}=V$, and

(E2) $e$ maps every line of $\Gamma$ onto a line of $\mathbb{P}(V)$.

The dimension of $(e, V)$ is $\operatorname{dim}(V)$. In the literature, this is sometimes called the vector dimension of the embedding to distinguish it from its projective dimension.

The collection $\mathcal{E}(\Gamma)$ of all full projective embeddings of $\Gamma$ over a division ring (or field) $\mathbb{F}$, is a category where a morphism between embeddings $\left(e_{1}, V_{1}\right)$ and $\left(e_{2}, V_{2}\right)$ is an $\mathbb{F}$-semilinear map $\tau: V_{1} \rightarrow V_{2}$ such that $e_{2}=\tau \circ e_{1}$. We sometimes indicate this 
by writing $e_{1} \geq e_{2}$. We have the usual notions of mono-, epi-, and isomorphisms. An embedding of $\Gamma$ is called absolutely universal or absolute if it is a source in $\mathcal{E}(\Gamma)$; if it exists, we denote it by $(\widetilde{e}, \widetilde{V})$. A source relative to $(e, V)$ always exists by a result due to Ronan [36]; it is called the embedding universal relative to $(e, V)$ and will be denoted $(\bar{e}, \bar{V})$.

An embedding $(e, V)$ of $\Gamma$ is called minimal if it is a sink in $\mathcal{E}(\Gamma)$ and minimal relative to $(e, V)$ if it is a sink relative to $(e, V)$; the latter will be denoted $(\underline{e}, \underline{V})$.

Let $(e, V)$ be a full projective embedding for $\Gamma$. For a point set $X \subseteq \mathcal{P}$, let $\langle X\rangle_{e}=$ $\langle e(X)\rangle_{V}$. The following is well-known and elementary.

\section{Lemma 1.2}

(a) If $U$ is a hyperplane of $V$, then $H=e^{-1}(U \cap e(\mathcal{P}))$ is a hyperplane of $\Gamma$.

(b) If $H$ is a maximal hyperplane of $\Gamma$, then $\langle H\rangle_{e}$ either equals $V$ or it is a hyperplane of $V$; in the latter case $e(H)=e(\mathcal{P}) \cap\langle H\rangle_{e}$.

The hyperplane $H$ in (a) is said to be induced by $U$ in $(e, V)$.

Buildings and Chevalley groups Every geometry we shall study in this paper is derived from a spherical building $\Delta$. The building $\Delta$ has spherical diagram $\mathrm{M}$ over the index set $I=\{1,2, \ldots, n\}$. We shall label $\mathrm{M}$ as in [7]. We shall think of $\Delta$ as a chamber system, also denoted $\Delta$, with a distance function $\delta: \Delta \times \Delta \rightarrow W$, where $\left(W,\left\{r_{i}\right\}_{i \in I}\right)$ is a Coxeter system of type $\mathrm{M}$; we shall use the terminology from $[37,53]$. Thus, for $i \in I$, we say that two chambers $c$ and $d$ are $i$-adjacent, and write $c \sim_{i} d$, if $\delta(c, d)=r_{i}$. For a subset $J \subseteq I$, the $I-J$-residue on $c$ is the collection of chambers $\left\{d \in \Delta \mid \delta(c, d) \in W_{I-J}\right\}$.

Sometimes it will be convenient to talk about $\Delta$ in terms of the associated incidence geometry $\mathcal{G}=\mathcal{G}(\Delta)$. This is a diagram geometry with a set of elements $\mathcal{E}=\mathcal{E}(\Delta)$, an incidence relation $\star$ and a type function typ: $\mathcal{E} \rightarrow I$; for $\mathcal{G}(\Delta)$ we shall use the terminology from [10, Chap. 1]. Thus, for $J \subseteq I$, a flag of type $J$ (or $J$-flag) in $\mathcal{G}$ is a collection $F=\left\{f_{j}\right\}_{j \in J}$ of pairwise incident elements and $\operatorname{Res}_{\Delta}(F)=\left\{e \in \mathcal{E}-F \mid e \star f_{j} \forall j \in J\right\}$ is the corresponding residue of type $I-J$ (or $I-J$-residue).

For buildings of finite rank these two viewpoints are equivalent (see e.g. [33, 49]); namely flags of type $J \subseteq I$ in the diagram geometry $\mathcal{G}$ correspond to residues of type $I-J$ in the chamber system $\Delta$ and incidence in the diagram geometry corresponds to non-empty intersection in the chamber system.

Whenever a spherical building $\Delta$ is Moufang, it has a strongly transitive automorphism group $G$ from which $\Delta$ can be recovered via a BN-pair in the manner described e.g. in [37, Chap. 5] or [53, Chap. 11]. Namely, pick an apartment $\Sigma$ and chamber $c \in \Sigma$, then $B=\operatorname{Stab}_{G}(c)$ and $N=\operatorname{Stab}_{G}(\Sigma)$ form a BN-pair for $G$. Conversely, given a BN-pair $(B, N)$, one can construct $\Delta$ by setting $\Delta=G / B$ and defining the $W$-valued distance function using the Bruhat decomposition of $G$ (see Sect. 4). For the spherical buildings under consideration in the present paper we take $G$ to be a Chevalley group of rank $n \geq 2$. In Sects. 2 and 3, $G$ can be of any twisted or untwisted type; in Sects. $4,5,6$, and 7, we require $G$ to be untwisted. We also assume $G$ to be the universal Chevalley group with diagram $M$ over $\mathbb{F}$, denoted $\bar{M}(\mathbb{F})$, although 
Table 1 Diagrams and groups

\begin{tabular}{|c|c|c|c|}
\hline$M$ & $G$ & $M$ & $G$ \\
\hline$A_{n}$ & $\mathrm{SL}_{n+1}(\mathbb{F})$ & $E_{6}$ & $\bar{E}_{6}(\mathbb{F})$ \\
\hline$B_{n}$ & $\operatorname{Spin}_{2 n+1},(\mathbb{F})$ & $E_{7}$ & $\bar{E}_{7}(\mathbb{F})$ \\
\hline$C_{n}$ & $\mathrm{Sp}_{2 n}(\mathbb{F})$ & $F_{4}$ & $\bar{F}_{4}(\mathbb{F})$ \\
\hline$D_{n}$ & $\operatorname{Spin}_{2 n}^{+}(\mathbb{F})$ & $G_{2}$ & $\bar{G}_{2}(\mathbb{F})$ \\
\hline
\end{tabular}

Untwisted universal Chevalley groups

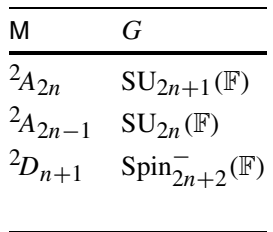

Some twisted Chevalley groups

this does not affect $\Delta$. In Sect. 9 we also include the twisted types ${ }^{2} A_{2 n},{ }^{2} A_{2 n+1}$, and ${ }^{2} D_{2 n+2}$. Finally, Sects. 8 and 10 only concern the $A_{n}$ case.

The correspondence between their diagrams $\mathrm{M}$, the (commutative) field of definition $\mathbb{F}$, and concrete universal Chevalley groups $G$ is given in Table 1.

Shadow spaces For any subset $L \subseteq I$, we denote by $\mathcal{S}_{L}(\Delta)=\left(\mathcal{P}_{L}(\Delta), \mathcal{L}_{L}(\Delta)\right)$ the $L$-shadow space of $\Delta$ (The term " $L$-Grassmannians" is also used, e.g. in [33]). This is the point-line geometry whose point set $\mathcal{P}_{L}(\Delta)$ consists of the flags of type $L$ of $\mathcal{G}(\Delta)$ and whose line set $\mathcal{L}_{L}(\Delta)$ consists of the collections of points incident to a flag of cotype $\{l\}$ for some $l \in L$ (We call $l$ the type of that line). This is a partial linear space (see e.g. [16, 18]). If $L=\{l\}$ for some $l \in I$, this is sometimes called the $l$-Grassmannian of $\Delta$. In case $\Delta$ is a building of type $A_{n}(\mathbb{F})$, this is the usual Grassmannian of the vector space $\mathbb{F}^{n+1}$. These single-node shadow spaces are also called Lie incidence geometries, see e.g. [17]. Note that if $L=\emptyset$, then $\mathcal{P}_{L}(\Delta)$ consists of a single point. At the other extreme, if $L=I$, then $\mathcal{P}_{L}(\Delta)$ is the collection of chambers of $\Delta$, and $\mathcal{L}_{L}(\Delta)$ is the collection of panels of $\Delta$. Fixing a non-empty subset $K \subseteq I$, we shall denote $\Gamma=\mathcal{S}_{K}(\Delta)=(\mathcal{P}, \mathcal{L}$ ) (so for this fixed geometry we drop the subscripts $K$ and $\Delta$ ).

Keeping $L$ as above, we define the $L$-shadow of an arbitrary set of chambers $X \subseteq \Delta$ to be

$$
\mathcal{P}_{\Delta, L}(X)=\left\{x \in \mathcal{P}_{L}(\Delta) \mid x \cap X \neq \emptyset\right\} .
$$

Here we view $x$ and $X$ as sets of chambers. We also set

$$
\begin{aligned}
& \mathcal{S}_{\Delta, L}(X)=\left(\mathcal{P}_{\Delta, L}(X), \mathcal{L}_{\Delta, L}(X)\right) \\
& \quad \text { where } \mathcal{L}_{\Delta, L}(X)=\left\{l \in \mathcal{L}_{L}(\Delta)|| l \cap \mathcal{P}_{\Delta, L}(X) \mid \geq 2\right\} .
\end{aligned}
$$

Note that $\mathcal{S}_{\Delta, L}(\Delta)=\mathcal{S}_{L}(\Delta)$.

We are mostly interested in the case where $X$ is a residue. Fix $J \subseteq I$. We let $\mathrm{M}_{J}$ denote the subdiagram of $\mathrm{M}$ induced on the nodes indexed by $J$. Then, $\left(W_{J},\left\{r_{j}\right\}_{j \in J}\right)$ is a Coxeter system of type $M_{J}$ (see e.g. [37, Corollary 2.14]).

Lemma 1.3 (Theorem 3.5 of [37]) Let $R$ be a $J$-residue of $\Delta$. Then $R$ is a building of type $\mathrm{M}_{J}$; the distance function is given by the restriction $\delta: R \times R \rightarrow W_{J}$. 
Lemma 1.4 (See also $[29,30])$ Let $R$ be a $J$-residue of $\Delta$.

(a) For any $L \subseteq I$, we have a natural isomorphism $\mathcal{S}_{\Delta, L}(R) \cong \mathcal{S}_{\Delta, L \cap J}(R)$.

(b) If $L_{1}, L_{2} \subseteq I$ are such that $L_{1} \cap J=L_{2} \cap J$, then we have a natural isomorphism $\mathcal{S}_{\Delta, L_{1}}(R) \cong \mathcal{S}_{\Delta, L_{2}}(R)$.

(c) If $L \subseteq I$, then $\mathcal{S}_{\Delta, L}(R) \cong \mathcal{S}_{L \cap J}(R)$.

Proof (a) Viewing the elements of $\mathcal{P}_{L}(\Delta)$ as sets of chambers, the isomorphism is induced by the map $x \mapsto x \cap R$, for $x \in \mathcal{P}_{\Delta, L}(R)$. For more details see the references [29, 30]. Part (b) is an immediate corollary to part (a). Part (c) follows from part (b) and the simple observation that $\mathcal{S}_{\Delta, L \cap J}(R) \cong \mathcal{S}_{L \cap J}(R)$.

Note that in part (c) of Lemma 1.4, $\mathcal{S}_{L \cap J}(R)$ is a shadow space of the building $R$ as in Lemma 1.3. Thus, part (c) allows us to view the $L \cap J$-shadow space of $R$ as a subspace of the $L$-shadow space of $\Delta$.

In addition to the references mentioned above, there are a few texts in preparation that deal extensively with shadow spaces: [11, 42].

\subsection{Main definitions}

In order to present the main results of this paper, we need some new definitions.

The opposition relation and the dual geometry Let $R$ be a residue of $\Delta$ of type $J \subseteq I$.

Conjugation by the longest word $w_{J}$ of the Coxeter system $\left(W_{J},\left\{r_{j}\right\}_{j \in J}\right)$ induces the opposition relation opp ${ }_{J}$ on the set $J: i \operatorname{opp}_{J} j$ if and only if $r_{i}=r_{j}^{w_{J}}$. We set

$$
\operatorname{opp}_{J}(L)=\left\{j \in J \mid j \operatorname{opp}_{J} l \text { for some } l \in L\right\} \text {. }
$$

In case $J=I$, we shall drop it from the notation.

The opposition relation between the chambers of $R$ is given by $x \operatorname{opp}_{R} y$ if and only if $\delta(x, y)=w_{J}$. We extend the opposition relation to residues $S$ and $T$ of $R$ by setting $S \operatorname{opp}_{R} T$ if every chamber of $S$ is opposite some chamber of $T$ and conversely.

Lemma 1.5 (See [53, Proposition 9.9 and Lemma 9.10]) Let $S$ be an L-residue of $R$ and let $t \in R$ be a chamber opposite some chamber of $S$. Let $T$ be the $\operatorname{opp}_{J}(L)$-residue on $t$. Then $T$ is the unique residue of $R$ opposite to $S$ and containing $t$.

Let $L \subseteq J$ and let $S$ be an $L$-residue of $R$. Define

$$
\begin{aligned}
\operatorname{opp}_{R}(S) & =\left\{c \in R \mid c \operatorname{opp}_{R} d \text { for some chamber } d \in S\right\} \\
\operatorname{near}_{R}(S) & =R-\operatorname{opp}_{R}(S)
\end{aligned}
$$

In case $R=\Delta$, we shall drop it from the notation. The following is immediate from Lemma 1.5. 
Corollary 1.1 Let $S$ be an L-residue of $R$. Then, $\operatorname{opp}_{R}(S)$ and $\operatorname{near}_{R}(S)$ can be partitioned into residues of type $\operatorname{opp}_{J}(L)$.

Recall that $\Gamma=(\mathcal{P}, \mathcal{L})$ is the $K$-shadow space of $\Delta$. The geometry dual to $\Gamma$ in $\Delta$ will be denoted $\Gamma^{*}=\left(\mathcal{P}^{*}, \mathcal{L}^{*}\right)$; it is equal to $\mathcal{S}_{\mathrm{opp}(K)}(\Delta)$. The geometry dual to $\mathcal{S}_{L}(R)$ in $R$ will be denoted $\mathcal{S}_{L}(R)^{*}$; it is equal to $\mathcal{S}_{\text {opp }_{J}(L)}(R)$.

We will call the points and lines of $\Gamma^{*}$ dual points and dual lines. We shall make use of the fact that $\Gamma^{* *}=\Gamma$. All statements made about $\Gamma$ can also be dualized and so we may and shall freely apply results stated for $\Gamma$ also to $\Gamma^{*}$.

Opposite and Far We briefly mention a concept related to the opposition relation. For residues $S$ and $T$ of $R$ we set

$S \operatorname{Far}_{R} T$ if and only if $s$ opp $_{R} t$ for some chambers $s \in S, t \in T$.

Using the correspondence $R \leftrightarrow \mathcal{G}(R)$, this then also defines a relation $\operatorname{Far}_{\mathcal{G}(R)}$ for flags of $\mathcal{G}(R)$.

Let $\operatorname{Far}_{\mathcal{G}(R)}(S)$ be the incidence system of all objects of $\mathcal{G}(R)$ 'far' from $S$ with incidence inherited from $\mathcal{G}(R)$. It is proved in [4] that this is a transversal geometry with a Buekenhout-Tits diagram whose flag system can be identified with the collection of all residues of $R$ intersecting $\operatorname{opp}_{R}(S)$ (as chamber sets) non-trivially. In particular, the set $\operatorname{opp}_{R}(S)$ is exactly the chamber system of $\operatorname{Far}_{\mathcal{G}(R)}(S)$. This means that two flags that are far from $S$ are incident exactly if they share a chamber that belongs to opp $\operatorname{op}_{R}(S)$. In particular, for any type set $L \subseteq J$, the subspace $\mathcal{S}_{R, L}\left(\operatorname{opp}_{R}(S)\right)$ of $\mathcal{S}_{L}(R)$ coincides with the $L$-shadow space defined by $\operatorname{Far}_{\mathcal{G}(R)}(S)$ in the obvious way.

Singular hyperplanes and polarized embeddings We continue the notation from above. There are two important geometric structures associated with $\operatorname{opp}_{R}(S)$ and near $_{R}(S)$.

$$
\begin{aligned}
\mathcal{P}_{R, L}\left(\operatorname{opp}_{R}(S)\right) & =\left\{x \in \mathcal{P}_{L}(R) \mid x \cap \operatorname{opp}_{R}(S) \neq \emptyset\right\}, \\
\mathcal{P}_{R, L}\left(\operatorname{near}_{R}(S)\right) & =\left\{x \in \mathcal{P}_{L}(R) \mid x \cap \operatorname{near}_{R}(S) \neq \emptyset\right\} .
\end{aligned}
$$

If $L$ is clear from the context, we shall omit it to unburden the notation. These sets are not always disjoint. However, we have the following simple observation.

Lemma 1.6 Let $R$ be a $J$-residue of $\Delta$. Let $L, L^{*} \subseteq J$ and let $S$ be a residue of type $J-L^{*}$ in $R$. Then $\mathcal{P}_{R, L}\left(\operatorname{near}_{R}(S)\right) \cap \mathcal{P}_{R, L}\left(\operatorname{opp}_{R}(S)\right)=\emptyset$ if and only if $\operatorname{opp}_{J}\left(L^{*}\right) \subseteq L$. In particular, the $K$-shadows of $\operatorname{opp}_{\Delta}\left(p^{*}\right)$ and near ${ }_{\Delta}\left(p^{*}\right)$ are disjoint subsets of $\mathcal{P}$ if $p^{*} \in \mathcal{P}^{*}$.

Proof We have $\mathcal{P}_{R, L}\left(\operatorname{near}_{R}(S)\right) \cap \mathcal{P}_{R, L}\left(\operatorname{opp}_{R}(S)\right)=\emptyset$ if and only if every $(J-L)$ residue of $R$ that meets $\operatorname{opp}_{R}(S)$ is entirely contained in $\operatorname{opp}_{R}(S)$. By Corollary 1.1, this happens if and only if $J-L \subseteq \operatorname{opp}_{J}\left(J-L^{*}\right)=J-\operatorname{opp}_{J}\left(L^{*}\right)$, that is, if $\operatorname{opp}_{J}\left(L^{*}\right) \subseteq L$. 
Definition 1.1 Given a $J$-residue $R$, a subset $L \subseteq J$ and a dual point $x^{*}$ in $\mathcal{P}_{L}(R)^{*}$, we shall define the following subspace of $\mathcal{S}_{L}(R)$ :

$$
H_{R, L}\left(x^{*}\right)=\mathcal{P}_{R, L}\left(\operatorname{near}_{R}\left(x^{*}\right)\right) .
$$

Note that by Lemma 1.6, we have $H_{R, L}\left(x^{*}\right)=\mathcal{P}_{L}(R)-\mathcal{P}_{R, L}\left(\operatorname{opp}_{R}\left(x^{*}\right)\right)$. In case $R=\Delta$ or $L=K$, we shall drop that subscript from the notation. As we shall see in Proposition 1.1, $H\left(x^{*}\right)$ is often a (maximal) hyperplane of $\Gamma$. Hyperplanes of the form $H\left(x^{*}\right)$ are called singular or attenuated.

Definition 1.2 We call a full projective embedding $(e, V)$ of $\Gamma$ polarized if every singular hyperplane is induced by $(e, V)$. In that case the polar radical of $e$ is the subspace

$$
\mathcal{R}_{e}=\bigcap_{p^{*} \in \Gamma^{*}}\left\langle H\left(p^{*}\right)\right\rangle_{e} .
$$

Remark 1.1 Our notion of "polarized" specializes to the notion of "polarized" defined in $[13,48]$ if we let $K=\{k\}$ refer to an end-node of a diagram M of type $B_{n}, C_{n}$, or ${ }^{2} A_{n}$. Since in those cases the map opp $I$ is equal to the identity it happens that $\Gamma^{*}=\Gamma$ in loc. cit. In the present paper we do not restrict ourselves to that situation. In particular, when $\mathrm{M}=A_{n}, D_{n}$ ( $n$ odd), or $E_{6}$ it may happen that $\Gamma^{*}$ and $\Gamma$ are different, if isomorphic, geometries.

\subsection{Main results and organization of the paper}

In Sects. 2 and 3 we consider a shadow space $\Gamma$ of a spherical Moufang building $\Delta$ associated with a twisted or untwisted Chevalley group $G$. The opposition relation gives us the right perspective on polarized embeddings of shadow spaces as we shall see; many fundamental properties of spherical buildings translate transparently into properties of polarized embeddings. In particular, the following is proved in Sect. 2. The case where $|K|=1$ was proved in $[3,5]$.

Proposition 1.1 For any dual point $p^{*}$ of $\Gamma^{*}$,

(a) $H\left(p^{*}\right)$ is a hyperplane of $\Gamma$, and

(b) $\mathcal{S}_{\Delta, K}\left(\operatorname{opp}_{\Delta}\left(p^{*}\right)\right)$ is a connected subgeometry of $\Gamma$, except if $\mathrm{M}_{n, K}(\mathbb{F})$ is one of the following:

(i) $G_{2,\{1\}}\left(\mathbb{F}_{2}\right)$ (1 denoting the short root), $G_{2,\{1,2\}}\left(\mathbb{F}_{2}\right), G_{2,\{1,2\}}\left(\mathbb{F}_{3}\right),{ }^{2} F_{4,\{1\}}\left(\mathbb{F}_{2}\right)$, ${ }^{2} F_{4,\{1,2\}}\left(\mathbb{F}_{2}\right)$,

(ii) $C_{n, K}\left(\mathbb{F}_{2}\right)$, or $F_{4, K}\left(\mathbb{F}_{2}\right)$, where $n \geq 2$ and $K$ contains both nodes of the double bond in the diagram.

As a consequence, except in cases (i) and (ii), $H\left(p^{*}\right)$ is a maximal subspace of $\Gamma$.

Thus, apart from a few exceptions, the geometry $\Gamma$ contains a maximal singular hyperplane for each point of the dual geometry $\Gamma^{*}$. As a step up to the major results of the paper, Theorem 1.1 says that singular hyperplanes of $\Gamma$ are "residually singular". As a consequence, polarized embeddings of $\Gamma$ are "residually polarized". 
Theorem 1.1 Let $\Delta$ be a spherical building with type set I and let $\Gamma$ be its $K$-shadow space for a non-empty set $K \subseteq I$. Let $R$ be a J-residue of $\Delta$.

(a) For any dual point $p^{* R}$ of $\mathcal{P}_{K \cap J}(R)^{*}$, there is some dual point $p^{*}$ of $\Gamma^{*}$ such that $H_{R, K \cap J}\left(p^{* R}\right)$ corresponds to $\mathcal{P}_{\Delta, K}(R) \cap H\left(p^{*}\right)$ under the isomorphism $\mathcal{S}_{K \cap J}(R) \cong \mathcal{S}_{\Delta, K}(R)$.

(b) If $(e, V)$ is a polarized embedding of $\Gamma$, then the isomorphism $\mathcal{S}_{K \cap J}(R) \cong$ $\mathcal{S}_{\Delta, K}(R)$ composed with e yields a full polarized embedding of $\mathcal{S}_{K \cap J}(R)$ into $\mathbb{P}\left(V_{R}\right)$; here $V_{R}=\left\langle e(x) \mid x \in \mathcal{P}_{\Delta, K}(R)\right\rangle$.

Theorem 1.1 is proved in Sect. 2.2. In short, part (b) follows directly from part (a). Part (a) follows from a fundamental relation between the opposition and projection maps of spherical buildings. This relation is conveyed in Theorem 2.1, which was proven in [4] to show that in a "Far away" geometry obtained from $\Gamma$ by removing the hyperplane $H\left(p^{*}\right)$, the residues are themselves "Far away" geometries and that, as a consequence, this Far away geometry has a Buekenhout-Tits diagram.

We also prove the following general property of polarized embeddings.

Proposition 1.2 If a point-line geometry $\Theta$ possesses a polarized embedding $e$ and an absolute embedding $\widetilde{e}$, then $\Theta$ possesses a unique minimal polarized embedding $\check{e}$. Namely we have $\check{e}=\widetilde{e} / \mathcal{R}_{\widetilde{e}}$.

We note that many shadow spaces of spherical buildings do have an absolute embedding $[6,31]$.

In Sects. 4 and 5 we consider the following setup. Let $\Delta$ be obtained from an untwisted Chevalley group $G$ over a field $\mathbb{F}$ (see Table 1). Fix a non-empty subset $K \subseteq I$ and let $\Gamma$ be the $K$-shadow space of $\Delta$. Let $V\left(\lambda_{K}\right)_{\mathbb{F}}$ be the weak Weyl module for $G$ with maximal vector $v^{+}$of highest weight $\lambda_{K}$, as defined in Sect. 4.2, and let $V^{0}=\mathbb{F} G v^{+}$. Let $p$ be the point of $\Gamma$ corresponding to the parabolic group stabilizing $v^{+}$. Then, the map

$$
\begin{aligned}
e_{K}: \Gamma & \rightarrow \mathbb{P}\left(V^{0}\right), \\
g p & \mapsto\left\langle g v^{+}\right\rangle
\end{aligned}
$$

defines a full projective embedding for $\Gamma$.

\section{Theorem 1.2}

(a) The embedding $e_{K}$ is polarized.

(b) The codomain $V^{0} / \mathcal{R}_{e_{K}}$ of the minimal polarized embedding relative to $e_{K}$ is the unique irreducible $G$-module $L\left(\lambda_{K}\right)_{\mathbb{F}}$ of highest weight $\lambda_{K}$.

The general idea of the proof is the following. In Sect. 5.2, following [27, 52, 54] we define a $\tau$-contravariant bilinear form $\beta$ on $V\left(\lambda_{K}\right)_{\mathbb{F}}$ with the property that $\beta\left(v^{+}, v^{+}\right)=1$. This means that there is an involution $\tau$ of $G$ that interchanges two opposite Borel groups $B^{+}$and $B^{-}$, such that, for $g \in G$ and $u, v \in V\left(\lambda_{K}\right)_{\mathbb{F}}$, we have

$$
\beta(g v, u)=\beta\left(v, g^{\tau}(u)\right) .
$$


This form is symmetric and non-degenerate on $V^{0}$ and has the property that weight spaces corresponding to distinct weights are orthogonal with respect to $\beta$. Then, in Sect. 5.3, we show that the subspace $\operatorname{ker}\left(\beta\left(v^{+},-\right)\right)$of $V^{0}$ induces a singular hyperplane $H\left(p^{*}\right)$ of $\Gamma$, for some dual point $p^{*}$ opposite to $p$, and part (a) follows by contravariance. From the preceding discussion it follows that the polar radical $\mathcal{R}_{e_{K}}$ coincides with the radical of $\beta$. It is known, and not hard to prove, that this radical is the unique largest submodule of $V^{0}$. Thus, the quotient $V^{0} / \mathcal{R}_{e_{K}}$ is an irreducible $G$-module of highest weight $\lambda_{K}$. Proposition 1.2 and Theorem 1.2 directly imply Theorem 1.3.

Theorem 1.3 Let $\Delta$ be a spherical building obtained from an untwisted Chevalley group and let $\Gamma$ be its $K$-shadow space, for some non-empty type set $K \subseteq$ I. If $\Gamma$ possesses an absolute embedding, then the unique irreducible $G$-module $L\left(\lambda_{K}\right)_{\mathbb{F}}$ of highest weight $\lambda_{K}$ affords the unique minimal polarized embedding for $\Gamma$.

Motivation for this paper The main motivation for the style in which this paper is written is to exhibit a connection between the geometry of shadow spaces of buildings and the representation theory of groups of Lie type. This builds on the connection between a number of results that seem to be known only to a handful of colleagues, but is, to the best of my knowledge, not written down anywhere. I have made an attempt to bring these results (on embeddings, buildings, Chevalley groups and their highest-weight representations) together in the hope that this makes this connection as well as the new results presented here accessible to a wider audience.

As for the results presented here themselves, let us mention at least two motivations. First of all, Theorem 1.2 allows us to "recognize" the fundamental weight modules geometrically as the minimal polarized embeddings of $\Gamma$. This opens the door to studying (certain) modular representations of Chevalley groups by geometric means. A pilot project formulated by Blok, Cardinali and Pasini to study the decomposition series of the Weyl modules of the symplectic groups from this perspective and reinterpret the results from $[2,34]$ is in progress.

Secondly, we would like to show that the notion of a polarized embedding of a shadow space is rather fundamental, as it relates directly to fundamental properties of the corresponding spherical buildings. This is for instance evidenced in Theorem 1.1.

\section{Problems}

(1) Modify the above results to include twisted Chevalley groups.

(2) Give a geometric proof that if a geometry $\Gamma$ with sufficiently transitive automorphism group $G$ has a minimal polarized embedding, then that embedding is irreducible for the automorphism group $G$, assuming that the automorphisms in $G$ lift to linear isomorphisms of that embedding.

(3) Use the above connection to find decomposition series of $G$-modules that afford embeddings for $\Gamma$.

In Sects. 6, 8, 9, and 10 we study polarized embeddings of various shadow spaces in detail. 


\section{Polarized embeddings of shadow spaces}

In this section we prove Proposition 1.1 which tells us when the set $H\left(p^{*}\right)$ is a maximal hyperplane of $\Gamma$. Then we prove Theorem 1.1 which says that a full polarized embedding of $\Gamma$ induces full polarized embeddings on each residue of $\Gamma$. Both are closely related to fundamental properties of buildings.

2.1 In which shadow spaces is $H\left(p^{*}\right)$ a maximal hyperplane?

Recall from Lemma 1.6 that, for a dual point $p^{*} \in \Gamma^{*}$, the subsets $H\left(p^{*}\right)=$ $\mathcal{P}_{\Delta, K}\left(\operatorname{near}\left(p^{*}\right)\right)$ and $\mathcal{P}_{\Delta, K}\left(\operatorname{opp}\left(p^{*}\right)\right)$ are disjoint subsets of $\Gamma$. We begin by proving Proposition 1.1.

Proof of Proposition 1.1 (a) Choose a line $l$. By definition this is the $K$-shadow of a $k$-panel $\pi$ for some $k \in K$. Either $\pi \subseteq$ near $\left(p^{*}\right)$ or $\pi \cap \operatorname{opp}\left(p^{*}\right) \neq \emptyset$. In the latter case $\operatorname{proj}_{\pi}\left(p^{*}\right)$ is the unique chamber of $\pi \cap$ near $\left(p^{*}\right)$. By Lemma 1.6, a point $q$ of $\mathcal{P}$ meeting $\pi$ is in $H\left(p^{*}\right)$ if and only if $q \cap \pi \in \operatorname{near}\left(p^{*}\right)$. Thus either one or all points of $l$ are in $H\left(p^{*}\right)$. This means that $H\left(p^{*}\right)$ is a hyperplane of $\Gamma$.

(b) It follows from $[1,3,4,9]$ that under the restrictions (i) and (ii), the collection $\operatorname{opp}\left(p^{*}\right)$ of chambers opposite to $p^{*}$ is connected as a chamber system. By Corollary $1.1, \mathcal{P}_{\Delta, K}\left(\operatorname{opp}\left(p^{*}\right)\right)$ is exactly the collection of points all of whose chambers are opposite to some chamber of $p^{*}$. Thus, connectedness of the chamber system implies connectedness of the subgeometry $\mathcal{S}_{\Delta, K}\left(\operatorname{opp}\left(p^{*}\right)\right)$ of $\Gamma$. That $H\left(p^{*}\right)$ is a maximal hyperplane of $\Gamma$ now follows from the above and Lemma 1.1.

\subsection{Polarized embeddings are residually polarized}

We prove Theorem 1.1, which says that singular hyperplanes of $\Gamma$ are "residually singular". That is, given a residue $R$ of $\Delta$, the $K$-shadow of a singular hyperplane intersected with the $K$-shadow $\mathcal{P}_{K}(R)$ of $R$, is a singular hyperplane, or all, of $\mathcal{P}_{K}(R)$. As a consequence, polarized embeddings of $\Gamma$ are "residually polarized". This result stems from Theorem 2.1, proved in [4], which describes the interaction of the opposition and projection maps, two of the most fundamental maps in the theory of spherical and twin-buildings. For convenience we quote this result here.

Theorem 2.1 (Lemmas 3.5 and 3.6 of [3] and Lemmas 6.2 and 6.4 of [4])

(a) Let $W$ be a Coxeter building of spherical type. Then for any two residues $R$ and $S$ of $W$ we have $\operatorname{opp}_{R}\left(\operatorname{proj}_{R}(S)\right)=\operatorname{proj}_{R}\left(\operatorname{opp}_{W}(S)\right)$.

(b) Let $R$ and $S$ be residues containing opposite chambers in the spherical building $\Delta$. Then the set of residues meeting $\operatorname{opp}_{\Delta}(S)$ and contained in $R$ equals the set of residues in $\operatorname{opp}_{R}\left(\operatorname{proj}_{R}(S)\right)$. In particular, every object in $\mathcal{G}(R)$ meets $\operatorname{opp}_{\Delta}(S)$ (as a set of chambers) if and only if $\operatorname{proj}_{R}(S)=R$, that is, if and only if $\operatorname{opp}_{I}(\operatorname{typ} S) \subseteq \operatorname{typ} R$.

We shall now prove Theorem 1.1. 
Proof of Theorem 1.1 (a) Let $p^{* R}$ be a dual point of $\mathcal{P}_{K \cap J}(R)^{*}$. For some apartment $\Sigma$ on $p^{* R}$, let $p^{*}$ be the unique dual point in $\Gamma^{*}$ meeting $\operatorname{opp}_{\Sigma}\left(\operatorname{opp}_{R \cap \Sigma}\left(p^{* R}\right)\right)$. Then, by Theorem 2.1(a) we have $p^{* R}=\operatorname{proj}_{R}\left(p^{*}\right)$ and so by Theorem 2.1(b) $p^{*}$ satisfies the claim.

(b) Clearly $\left(e, V_{R}\right)$ is a full embedding for $\mathcal{S}_{K}(R)$. Now consider a dual point $p^{* R}$ of $\mathcal{P}_{K \cap J}(R)^{*}$ and let $\Sigma$ and $p^{*}$ be as in (a). Moreover, let $p$ be the point of $\Gamma$ meeting opp $\operatorname{op}_{R \cap \Sigma}\left(p^{* R}\right) \subseteq \operatorname{opp}_{\Sigma}\left(p^{*}\right)$. Then, $p \notin H\left(p^{*}\right)$ and since $H\left(p^{*}\right)$ is induced by $V$, also $e(p) \notin\left\langle H\left(p^{*}\right)\right\rangle_{e}$. It follows that also $H_{R}=V_{R} \cap\left\langle H\left(p^{*}\right)\right\rangle_{e}$ is a proper hyperplane of $V_{R}$.

To see that $H_{R}$ induces $H_{R}\left(p^{* R}\right)$, note that, by (a) $\left\langle H_{R}\left(p^{* R}\right)\right\rangle_{e} \leq H_{R}$. As both the former and the latter are hyperplanes of $V_{R}$, we must have equality. That is, $H_{R}\left(p^{* R}\right)$ is induced by $\left(e, V_{R}\right)$.

\section{Covers and quotients of polarized embeddings}

In this section we obtain some general properties of polarized embeddings. In particular we consider absolute and minimal polarized embeddings. In [13] several of these results were obtained for dual polar spaces. As it turns out, many of these can be generalized to arbitrary shadow spaces.

\subsection{The polar radical}

We first consider an arbitrary point-line geometry $\Gamma$ and a full projective embedding $(e, V)$.

Definition 3.1 Call $R \leq V$ a factoring subspace for $(e, V)$ if

(QE1) $R \cap e(p)=\{0\}$ for every point $p$ of $\Gamma$, and

(QE2) for any two distinct points $p, q \in \Gamma$ we have $\langle R, e(p)\rangle_{V} \neq\langle R, e(q)\rangle_{V}$.

For any factoring subspace $R \leq V$, we define the mapping $e / R$, called the quotient of e over $R$, as follows:

$$
\begin{aligned}
e / R: \Gamma & \rightarrow \mathbb{P}(V / R), \\
p & \mapsto\langle R, e(p)\rangle .
\end{aligned}
$$

The following are immediate (cf. [13]):

Lemma 3.1 Let $(e, V)$ be a projective embedding of a point-line geometry $\Gamma$ and let $R$ be a factoring subspace. If e is a full embedding, then so is $e / R$ and if $e / R$ is polarized, then so is $e$.

Corollary 3.1 If $\Gamma$ has a polarized embedding, then its absolutely universal embedding, if it exists, is also polarized. 
We now return to the standard situation of the paper, where $\Gamma$ is the $K$ shadow space of the spherical building $\Delta$. Recall from Definition 1.2 that the polar radical of a polarized embedding $(e, V)$ is the subspace

$$
\mathcal{R}_{e}=\bigcap_{p^{*} \in \Gamma^{*}}\left\langle H\left(p^{*}\right)\right\rangle_{e} .
$$

Any subspace of $\mathcal{R}_{e}$ is called a radical subspace. The significance of radical subspaces is given by the following result.

Proposition 3.1 Let $(e, V)$ be a polarized embedding of a shadow space $\Gamma$ and let $R \leq V$.

(a) If $R \leq \mathcal{R}_{e}$, then $R$ is a factoring subspace and $e / R$ is polarized.

(b) If $R$ is a factoring subspace such that e/R is polarized, then $R \leq \mathcal{R}_{e}$.

(c) $e / \mathcal{R}_{e}$ is the minimal polarized quotient of $e$.

Proof (a) (QE1) Let $p$ be any point of $\Gamma$. Then it is opposite some dual point $p^{*} \in$ $\Gamma^{*}$ so that $p \notin H\left(p^{*}\right)$. Since $H\left(p^{*}\right)$ is induced by $e$ this means that $e(p) \cap R \subseteq$ $e(p) \cap\left\langle H\left(p^{*}\right)\right\rangle_{e}=\{0\}$ and so $(\mathrm{QE} 1)$ is satisfied.

(QE2) Clearly $\langle R, e(p)\rangle \leq\left\langle\mathcal{R}_{e}, e(p)\right\rangle \leq\left\langle H\left(x^{*}\right)\right\rangle_{e}$ for any dual point $x^{*}$ satisfying $p \in H\left(x^{*}\right)$. Now let $p$ and $q$ be distinct points of $\Gamma$. Pick an apartment $\Sigma$ on $p$ and $q$ and let $p^{*}=\operatorname{opp}_{\Sigma}(p)$ and $q^{*}=\operatorname{opp}_{\Sigma}(q)$. Then $p^{*}$ is not opposite to $q$ and $q^{*}$ is not opposite to $p$. Hence, $e(q) \notin \bigcap_{x^{*} \in H^{*}(p)}\left\langle H\left(x^{*}\right)\right\rangle_{e} \geq\left\langle\mathcal{R}_{e}, e(p)\right\rangle \geq\langle R, e(p)\rangle$ and $e(p) \notin \bigcap_{y^{*} \in H^{*}(q)}\left\langle H\left(y^{*}\right)\right\rangle_{e} \geq\left\langle\mathcal{R}_{e}, e(q)\right\rangle \geq\langle R, e(q)\rangle$. Here $H^{*}(x)$ denotes the hyperplane of $\Gamma^{*}$ consisting of dual points not opposite to the point $x$. This proves (QE2). By Lemma $3.1 e / R$ is again a full projective embedding.

Moreover, since $R \leq\left\langle H\left(p^{*}\right)\right\rangle_{e}$ for every dual point $p^{*}$ and $\left\langle H\left(p^{*}\right)\right\rangle_{e}$ is a hyperplane of $V,\left\langle H\left(p^{*}\right)\right\rangle_{e / R}$ is again a hyperplane of $V / R$. That is, $e / R$ is again polarized.

(b) Suppose $R \not \leq \mathcal{R}_{e}$. Then there is some dual point $p^{*}$ such that $R \not \leq\left\langle H\left(p^{*}\right)\right\rangle_{e}$. As a consequence, $\left\langle(e / R)\left(H\left(p^{*}\right)\right)\right\rangle=V / R$, contradicting that $e / R$ is polarized.

(c) This follows immediately from (b).

\subsection{Absolutely universal embeddings}

Combining Corollary 3.1 with Theorem 1.2 we find the following.

Corollary 3.2 Let $\Delta$ be a spherical building obtained from an untwisted Chevalley group and let $\Gamma$ be its $K$-shadow space, for some non-empty type set $K \subseteq I$. Then, the absolutely universal embedding of $\Gamma$, if it exists, is polarized.

In [31] and [6] it is shown that for many buildings $\Delta$ and type sets $K$, the shadow space $\Gamma$ does possess an absolutely universal embedding. So Corollary 3.2 ensures that this embedding is polarized whenever that building is obtained from an untwisted Chevalley group.

For several special single-node shadow spaces $\Gamma$ even more is known; namely, that a particular embedding induces all hyperplanes of $\Gamma$. Then, since Veldkamp lines 
Table 2 Shadow spaces whose universal embedding affords all hyperplanes

\begin{tabular}{ll}
\hline Type of geometry & Source \\
\hline $\begin{array}{l}1 \text { Desarguesian projective spaces of finite rank. } \\
2 \text { Embeddable non-degenerate polar spaces of rank at least } 3 \text { and embeddable }\end{array}$ & {$[51]$} \\
$\begin{array}{l}\text { generalized quadrangles which do not possess ovoids. } \\
3 \text { All embeddable point-line geometries with three points per line. }\end{array}$ & {$[36]$} \\
4 The Grassmannian of projective lines over a field $\left(A_{n, 2}(\mathbb{F}), n>3, \mathbb{F}\right.$ a field $)$ and low & {$[19,21]$} \\
rank geometries $D_{5,5}$ and $E_{6,1}$. & {$[38]$} \\
5 All embeddable Grassmannians $A_{n, k}(\mathbb{F}), n>2,1<k<n-1, \mathbb{F}$ a field. & {$[39]$} \\
6 All half-spin geometries $D_{n, n}(\mathbb{F}), n>4$. & {$[43]$} \\
7 All orthogonal spin geometries $B_{n, n}(\mathbb{F})$, where $\mathbb{F}$ is such that $B_{2,2}(\mathbb{F})$ has no ovoids. & {$[41]$} \\
8 The exceptional geometry $E_{7,7}(\mathbb{F}), \mathbb{F}$ a field. &
\end{tabular}

exist in those cases (see [40]), that particular embedding is the absolutely universal embedding. Clearly, in those cases the absolute embedding is polarized. Table 2 lists some of these shadow spaces. The entries 1-6 come from [43]. In this table we find all embeddable non-degenerate polar spaces, (including those coming from untwisted Chevalley groups with diagrams $\mathrm{M}_{n, k}$ equal to one of $\left.B_{n, 1}, C_{n, 1}, D_{n, 1}\right)$. Note that all minuscule weight geometries of untwisted Chevalley groups are present: they are the geometries of type $A_{n, k}$ (any $\left.k\right), B_{n, n}(n \geq 2), C_{n, 1}(n \geq 3), D_{n, 1}, D_{n, n-1}, D_{n, n}$ $(n \geq 4), E_{6,1}$ and $E_{6,6}$, and $E_{7,7}$ (for a definition of a minuscule weight see Sect. 6).

\subsection{Minimal polarized embeddings}

We first prove Proposition 1.2.

Proof of Proposition 1.2 Let $e$ be any polarized embedding of the point-line geometry $\Theta$. Let $\widetilde{\mathcal{R}}=\mathcal{R}_{\widetilde{\widetilde{e}}}$ be the radical of the absolute embedding of $\Theta$ and set $\check{e}=\tilde{e} / \widetilde{\mathcal{R}}$ and $\check{V}=\widetilde{V} / \widetilde{\mathcal{R}}$. By Corollary 3.1 $\widetilde{e}$ is polarized as well. Since $\widetilde{e}$ is absolute, there is some subspace $R$ such that $\widetilde{e} / R=e$. Since $e$ is polarized, it follows from Proposition 3.1 that $R \leq \widetilde{\mathcal{R}}$. The canonical maps corresponding to the inclusions $\{0\} \leq R \leq \widetilde{\mathcal{R}}$ yield morphisms $\widetilde{e} \rightarrow e \rightarrow \check{e}$. Since $\widetilde{e}$ does not depend on $e, \check{e}$ is the unique minimal polarized embedding of $\Theta$.

We return to the situation where $\Gamma$ is the $K$-shadow space of a spherical building $\Delta$ for some non-empty subset $K$ of $I$. In Theorem 1.1 it was shown that any full polarized embedding $e$ of $\Gamma$ induces a full polarized embedding on each of the residues of $\Gamma$. The next result shows that the same is true if we replace "full polarized" by "minimal full polarized".

Theorem 3.1 A minimal polarized embedding of $\Gamma$ induces a minimal polarized embedding on each residue of $\Gamma$. 
Proof Let $(e, V)$ be a minimal polarized embedding for $\Gamma$. That is, $\mathcal{R}_{e}=\{0\}$. Let $R$ be a $J$-residue of $\Delta$. Identify $\mathcal{S}_{K \cap J}(R) \cong \mathcal{S}_{\Delta, K}(R)$. Let $\left(e^{\prime}, V^{\prime}\right)$ be the embedding induced by $e$ on $\mathcal{S}_{\Delta, K}(R)$, that is, $e^{\prime}$ is the restriction of $e$ to $\mathcal{P}_{\Delta, K}(R)$, with codomain $V^{\prime}=\left\langle\mathcal{P}_{\Delta, K}(R)\right\rangle_{e}$. By Theorem 1.1, $\left(e^{\prime}, V^{\prime}\right)$ is polarized. It suffices to show that the polar radical $\mathcal{R}_{e^{\prime}}$ is trivial.

Instead we prove a slightly stronger claim from which the result immediately follows. Namely

$$
\mathcal{R}_{e} \cap V^{\prime}=\mathcal{R}_{e^{\prime}}
$$

First of all, if $x^{*}$ is not opposite to any point of $\mathcal{P}_{\Delta, K}(R)$, then $\left\langle H\left(x^{*}\right)\right\rangle_{e} \geq$ $e\left(\left\langle\mathcal{P}_{\Delta, K}(R)\right\rangle\right)=V^{\prime}$ and so

$$
\mathcal{R}_{e} \cap V^{\prime}=\bigcap_{x^{*} \in \mathcal{P}^{*}}\left\langle H\left(x^{*}\right)\right\rangle_{e} \cap V^{\prime}=\bigcap_{x^{*}}\left\langle H\left(x^{*}\right)\right\rangle_{e} \cap V^{\prime}
$$

where $x^{*}$ runs over all dual points in $\mathcal{P}^{*}$ that are opposite some point of $\mathcal{P}_{K}(R)$.

Now suppose that $x^{*}$ is opposite some point $x$ of $\mathcal{P}_{\Delta, K}(R)$. By Theorem 2.1 part (b), we have $H\left(x^{*}\right) \cap \mathcal{P}_{\Delta, K}(R)=H_{R, K \cap J}\left(x^{* R}\right)$, where $x^{* R}=\operatorname{proj}_{R}\left(x^{*}\right)$ and by part (a) of Theorem 2.1, $x^{* R} \in \mathcal{P}_{K \cap J}(R)^{*}$. Moreover, by Theorem 1.1(a), as $x^{*}$ runs over all dual points in $\mathcal{P}^{*}, x^{* R}$ runs over all dual points in $\mathcal{P}_{K \cap J}(R)^{*}$. Combining this with the previous equality, we find

$$
\mathcal{R}_{e} \cap V^{\prime}=\bigcap_{x^{* R} \in \mathcal{P}_{K \cap J}(R)^{*}}\left\langle H_{R, K \cap J}\left(x^{* R}\right)\right\rangle_{e^{\prime}}=\mathcal{R}_{e^{\prime}} .
$$

\section{The Weyl embedding}

Let $\Gamma$ be the $K$-shadow space of the spherical building $\Delta$, for some subset $K$ of $I$. We prove Theorem 4.2, stated below, which says that if $\Delta$ is obtained from an untwisted Chevalley group $G$, then $\Gamma$ can be embedded into a subspace of the Weyl module of suitably chosen highest weight. We shall call this embedding the Weyl embedding. A special case, where $\Gamma$ is a single node shadow space was considered in $[3,5]$.

\section{1 $\Gamma$ obtained from a $B N$-pair of $G$}

We shall assume that $\Delta$ is obtained from the universal Chevalley group $G=\overline{\mathrm{M}}(\mathbb{F})$ over the field $\mathbb{F}$ with Dynkin diagram $\mathrm{M}$ over the set $I=\{1,2, \ldots, n\}$ via its thick $B N$-pair in the canonical way (see $[37,50,53]$ ). That is, we let $\Delta$ be the chamber system whose set of chambers is $G / B$ and in which the distance function is given by

$$
\begin{aligned}
& \delta: \Delta \times \Delta \rightarrow W=N / H, \quad \text { where } H=B \cap N, \\
& (g B, h B) \mapsto w, \quad \text { where } B g^{-1} h B=B w B .
\end{aligned}
$$


Let $c$ be the chamber corresponding to $B$ and let $\Sigma$ be the apartment corresponding to the collection of $B$ cosets $N B$. For every $J \subseteq I$, let $P_{J}$ be the standard parabolic subgroup of $G$ of type $J$. This is the stabilizer of the $J$-residue and the $I-J$-flag on $c$.

We now construct the $K$-shadow space $\Gamma=(\mathcal{P}, \mathcal{L})$ of $\Delta$ as follows:

$$
\begin{aligned}
\mathcal{P} & =G / P_{I-K}, \\
\mathcal{L} & =\bigcup_{k \in K} \mathcal{L}_{k}, \\
& \text { where } \mathcal{L}_{k}=\left\{\left\{g h P_{I-K} \mid h \in P_{k}\right\} \mid g \in G\right\} \text { for each } k \in K .
\end{aligned}
$$

So $\mathcal{L}$ is the union of $G$-orbits of the "fundamental" lines $\left\{h P_{I-K} \mid h \in P_{\{k\}}\right\}$. Incidence is symmetrized containment.

Remark 4.1 For the above construction there is no need to restrict to the case where $G$ is universal. As is proved for instance in $[45, \S 3]$ if $G^{\prime}$ is any other Chevalley group of type $\mathrm{M}$ over $\mathbb{F}$, then $G^{\prime}$ is a central quotient of the universal Chevalley group $G$. Since the center of $G$ is contained in $B \cap N$, replacing $G$ by $G^{\prime}$ in the above construction yields canonically isomorphic $\Delta$ and $\Gamma$.

\subsection{The Weyl module $V(\lambda)_{\mathbb{F}}^{0}$}

Given a weight $\lambda$ of the complex semi-simple Lie algebra of type $M$ and a field $\mathbb{F}$, we construct the Weyl module $\mathbf{V}=V(\lambda)_{\mathbb{F}}^{0}$ and an associated Chevalley group $G_{\lambda}(\mathbb{F})$ along the lines of [27, Chap. 4]. Another, ultimately equivalent, approach was taken in [54].

The Kostant $\mathbb{Z}$-form of the universal enveloping algebra Let $\mathfrak{g}_{\mathbb{C}}$ be the semisimple Lie algebra with Dynkin diagram $\mathrm{M}$ indexed by $I=\{1,2, \ldots, n\}$ over $\mathbb{C}$. Let $\mathfrak{U}=\mathfrak{U}\left(\mathfrak{g}_{\mathbb{C}}\right)$ be its universal enveloping algebra. Fix a Cartan subalgebra $\mathfrak{h}_{\mathbb{C}}$ of $\mathfrak{g}_{\mathbb{C}}$ and a choice of positive roots $\Phi^{+}$with fundamental roots $\alpha_{1}, \ldots, \alpha_{n}$, along with a Chevalley basis $\mathrm{C}$ in $\mathfrak{g}_{\mathbb{C}}$ consisting of co-roots $H_{1}, \ldots, H_{n} \in \mathfrak{h}_{\mathbb{C}}, H_{i}$ being the co-root associated with $\alpha_{i}$, as well as a positive root vector $X_{\alpha}$ and a negative root vector $Y_{\alpha}$ for each $\alpha \in \Phi^{+}$. Let $\mathfrak{g}_{\mathbb{Z}}$ be the $\mathbb{Z}$-span of this Chevalley basis C; it is stable under the Kostant $\mathbb{Z}$-form $\mathfrak{U}_{\mathbb{Z}}$ of $\mathfrak{U}$, which is generated by 1 along with all products $H_{i}\left(H_{i}-1\right) \cdots\left(H_{i}-a+1\right) / a$ ! and all $X_{\alpha}^{a} / a$ ! and $Y_{\alpha}^{a} / a$ !. Then we can find a triangular decomposition $\mathfrak{U}=\mathfrak{U}^{-} \mathfrak{U}^{0} \mathfrak{U}^{+}$relative to a fixed ordering of the set of all positive roots $\alpha_{1}, \ldots, \alpha_{r}$ compatible with $\mathfrak{U}_{\mathbb{Z}}$. For example, $\mathfrak{U}_{\mathbb{Z}}^{+}$has as a $\mathbb{Z}$-basis all products $X_{\alpha_{1}}^{a_{1}} /\left(a_{1} !\right) \cdots X_{\alpha_{r}}^{a_{r}} /\left(a_{r} !\right)$.

The Verma module $M(\lambda)_{\mathbb{C}}$ and admissible lattices Let $\mathrm{E}$ be the real subspace of the dual space $\mathfrak{h}_{\mathbb{C}}^{*}$ of $\mathfrak{h}_{\mathbb{C}}$ spanned by the root system $\Phi$. The set $\Lambda_{w}=\{\lambda \in \mathrm{E} \mid$ $\left.\lambda\left(H_{j}\right) \in \mathbb{Z} \forall j \in I\right\}$ forms a lattice and is called the weight lattice. It has a $\mathbb{Z}$-basis $\left\{\lambda_{i} \mid i \in I\right\}$, where $\lambda_{i}$ is the fundamental dominant weight associated with the $i$ th node of M (labeled as in [8]) and has the property that $\lambda_{i}\left(H_{j}\right)=\delta_{i j}$ for all $i, j \in I$. Roots are also weights and, accordingly, the root lattice $\Lambda_{r} \leq \Lambda_{w}$ is the $\mathbb{Z}$-span of the 
fundamental system $\Pi=\left\{\alpha_{i} \mid i \in I\right\}$. There is a natural ordering on weights given by setting $\mu \prec v$, whenever $v-\mu$ is a sum of positive roots $\left(\mu, v \in \Lambda_{w}\right)$.

Now given any weight $\lambda$, there is a $\mathfrak{g}_{\mathbb{C}}$-module $M(\lambda) \mathbb{C}$, called the Verma module, having a unique maximal submodule $M^{\prime}$ and unique simple quotient $V(\lambda) \mathbb{C}$ (also denoted $L(\lambda)_{\mathbb{C}}$ ) (see e.g. [15, Chap. 10]). It is a well-known result that this simple module is finite-dimensional if and only if $\lambda$ is dominant. That is, $\lambda=\sum_{i \in I} n_{i} \lambda_{i}$, where $n_{i} \in \mathbb{N}$ are not all zero. Since this is the case we're interested in, we shall henceforth assume that $\lambda$ is dominant. Of particular importance for us are the following weights

$$
\lambda_{K}=\sum_{k \in K} \lambda_{k} \quad \text { for some non-empty subset } K \subseteq I \text {. }
$$

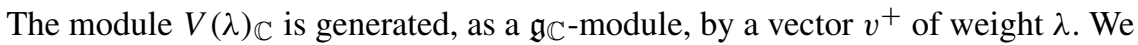
pause here to insert an important observation on weight spaces of a finite-dimensional

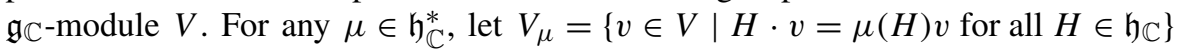
be the weight space of $V$ corresponding to $\mu$.The weight-lattice of $V$ is defined by $\Lambda(V)=\left\{\mu \in \mathfrak{h}_{\mathbb{C}}^{*} \mid V_{\mu} \neq 0\right\}$. It satisfies $\Lambda_{r} \leq \Lambda(V) \leq \Lambda_{w}[45, \S 3]$. We shall denote $\Lambda(\lambda)=\Lambda\left(V(\lambda)_{\mathbb{C}}\right)$. Now $\lambda$ is the highest weight of $V(\lambda)_{\mathbb{C}}$ in the sense that it maximal in $\Lambda(\lambda)$ with respect to the ordering $\prec$. Accordingly we call $v^{+}$a maximal or highest weight vector.

Following [25, Chap. 27] an admissible lattice in $V(\lambda) \mathbb{C}$ is a finitely generated $\mathbb{Z}$-submodule of $V(\lambda)_{\mathbb{C}}$ that spans $V(\lambda)_{\mathbb{C}}$ over $\mathbb{C}$, has $\mathbb{Z}$-rank at most $\operatorname{dim}_{\mathbb{C}}\left(V(\lambda)_{\mathbb{C}}\right)$ and is invariant under $\mathfrak{U}_{\mathbb{Z}}$. The minimal choice would be $A_{\min }=V(\lambda)_{\mathbb{Z}}=\mathfrak{U}_{\mathbb{Z}} v^{+}$. There is also a unique maximal admissible lattice, denoted $A_{\max }$; it can be obtained as the dual of a minimal admissible lattice in the dual module (see also Example 5.1). Any other admissible lattice $A$ for $V(\lambda) \mathbb{C}$ satisfies $A_{\min } \leq A \leq A_{\max }$. For any $\mu \in$ $\Lambda(\lambda)$, we set $A_{\mu}=A \cap\left(V(\lambda)_{\mathbb{C}}\right)_{\mu}$.

In the remainder of this section, unless otherwise specified, we shall work with the minimal admissible lattice $A_{\min }=V(\lambda)_{\mathbb{Z}}$.

\section{Proposition 4.1}

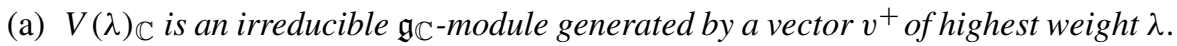

(b) $V(\lambda)_{\mathbb{C}}$ is the direct sum of its weight spaces. That is, $V(\lambda)_{\mathbb{C}}=\bigoplus_{\mu \in \Lambda(\lambda)} V(\lambda)_{\mathbb{C}, \mu}$, where $V(\lambda)_{\mathbb{C}, \lambda}=\mathbb{C} v^{+}$.

Let $A$ be any admissible lattice in $V(\lambda)_{\mathbb{C}}$ and let $A_{\mu}=A \cap V(\lambda)_{\mathbb{C}, \mu}$.

(c) We have $A=\bigoplus_{\mu \in \Lambda(\lambda)} A_{\mu}$ and $A_{\lambda}=\mathbb{Z} v^{+}$for suitable choice of $v^{+}$.

(d) For each weight $\mu$ of $V(\lambda)_{\mathbb{C}}, A_{\mu}$ spans $V(\lambda)_{\mathbb{C}, \mu}$.

Proof Proofs can be found in the following references. (a) [15, Chap. 10], [25, Chap. 21]; (b) [15, Chap. 2], [25, Chap. 20]; (c) [25, Chap. 27]. In particular Theorem 27.1 and its proof. (d) Clearly, for each weight $\mu$ of $V(\lambda)_{\mathbb{C}}$, we have $\left\langle A_{\mu}\right\rangle \leq V(\lambda)_{\mathbb{C}, \mu}$. Now note that, by definition, $A$ spans $V(\lambda)_{\mathbb{C}}$. Combining (b) and (c), we see that $A$ spans $V(\lambda)_{\mathbb{C}}$ if and only if, for each $\mu,\left\langle A_{\mu}\right\rangle=V(\lambda)_{\mathbb{C}, \mu}$.

As an example the minimal admissible lattices for $\mathfrak{g}_{\mathbb{C}}=\mathfrak{s l}_{2}(\mathbb{C})$ are described in Lemma 4.1. 
Lemma 4.1 [25, Chap. 7] Let $\mathfrak{g}_{\mathbb{C}}=\mathfrak{s l}_{2}(\mathbb{C})$ and let $\lambda=m \lambda_{1}$ for some $m \in \mathbb{N}$. Also write $\alpha=\alpha_{1}$. Then, we have

(a) $A_{\min }=V(\lambda)_{\mathbb{Z}}$ is a free $\mathbb{Z}$-module with basis $\left\{v_{0}, v_{1}, \ldots, v_{m}\right\}$, where $v_{i}=$ $\left(Y_{\alpha}^{i} / i !\right) v^{+}$.

(b) Setting $v_{-1}=v_{m+1}=0$, we have

$$
H_{\alpha} v_{i}=(m-2 i) v_{i}, \quad X_{\alpha} v_{i}=(m-i+1) v_{i-1}, \quad Y_{\alpha} v_{i}=(i+1) v_{i+1} .
$$

(c) The maximal lattice $A_{\max }$ corresponding to $A_{\min }$ is a free $\mathbb{Z}$-module with basis $\left\{f_{0}, f_{1}, \ldots, f_{m}\right\}$, where the action is given by

$$
H_{\alpha} f_{i}=(m-2 i) f_{i}, \quad X_{\alpha} f_{i}=i f_{i-1}, \quad Y_{\alpha} f_{i}=(m+1-i) f_{i+1} .
$$

Here $f_{m+1}=f_{-1}=0$. In fact we have $v_{i}=\left(\begin{array}{c}m \\ i\end{array}\right) f_{i}$ for all $i$.

Passage to an arbitrary field $\mathbb{F}$ We now pass to an arbitrary field $\mathbb{F}$. We follow Chap. 27 from [25]. Let $\mathfrak{g}(\lambda)_{\mathbb{Z}}$ be the stabilizer in $\mathfrak{g}_{\mathbb{C}}$ of $V(\lambda)_{\mathbb{Z}}$; it contains the $\mathbb{Z}$-span of the Chevalley basis $C$ for $\mathfrak{g}_{\mathbb{C}}$. In fact

$$
\mathfrak{g}(\lambda)_{\mathbb{Z}}=\mathfrak{h}(\lambda)_{\mathbb{Z}} \oplus \bigoplus_{\alpha \in \Phi^{+}} \mathbb{Z} X_{\alpha} \oplus \bigoplus_{\alpha \in \Phi^{+}} \mathbb{Z} Y_{\alpha},
$$

where $\mathfrak{h}(\lambda)_{\mathbb{Z}}=\{H \in \mathfrak{h} \mid \forall \mu \in \Lambda(\lambda): \mu(H) \in \mathbb{Z}\}$. Now we pass to the field $\mathbb{F}$ by setting

$$
\begin{aligned}
V(\lambda)_{\mathbb{F}} & =\mathbb{F} \otimes_{\mathbb{Z}} V(\lambda)_{\mathbb{Z}}, \\
\mathfrak{g}(\lambda)_{\mathbb{F}} & =\mathbb{F} \otimes_{\mathbb{Z}} \mathfrak{g}(\lambda)_{\mathbb{Z}} .
\end{aligned}
$$

The module $V(\lambda)_{\mathbb{F}}$ shall be called the weak Weyl module of highest weight $\lambda$ over $\mathbb{F}$.

Remark 4.2 The construction of $\mathfrak{g}(\lambda)_{\mathbb{Z}}, \mathfrak{h}(\lambda)_{\mathbb{Z}}$ and $V(\lambda)_{\mathbb{F}}$ can be done using any admissible lattice $A$ instead of the minimal lattice $V(\lambda)_{\mathbb{Z}}$. It is shown in loc. cit. that up to isomorphism, $\mathfrak{g}(\lambda)_{\mathbb{Z}}$ and $\mathfrak{h}(\lambda)_{\mathbb{Z}}$ only depend on $V(\lambda)_{\mathbb{C}}$ (or in fact $\Lambda(\lambda)$ ), not on the choice of $A$. However, it does affect the $\mathfrak{g}(\lambda)_{\mathbb{F}}$-action on $V(\lambda)_{\mathbb{F}}$, as one can deduce from Lemma 4.1 .

Remark 4.3 For any weight $\mu \in \Lambda(\lambda)$ and $a \in A_{\mu}$, we have $\left(1 \otimes H_{i}\right) \cdot(1 \otimes a)=$ $\mu\left(H_{i}\right) \otimes a$, where $\mu\left(H_{i}\right) \in \mathbb{F}$. Thus, if $\mathbb{F}$ has characteristic 0 , distinct weights of $V(\lambda)_{\mathbb{C}}$ induce distinct weights of $V(\lambda)_{\mathbb{F}}$. On the other hand, if $\mathbb{F}$ has positive characteristic $p$, then it may occur for distinct weights $\mu$ and $v$ of $V(\lambda)_{\mathbb{C}}$ that $\mu\left(H_{i}\right) \equiv v\left(H_{i}\right)(\bmod p)$ for all $i=1,2, \ldots, n$. In particular $\mathbb{F} \otimes_{\mathbb{Z}} A_{\mu}$ and $\mathbb{F} \otimes_{\mathbb{Z}} A_{v}$ belong to the same weight space of $V(\lambda)_{\mathbb{F}}$.

Definition 4.1 For each weight $\mu \in \Lambda(\lambda)$, we shall set $V(\lambda)_{\mathbb{F}, \mu}=\mathbb{F} \otimes_{\mathbb{Z}} A_{\mu}$ and call this the reduced $\mu$-weight space.

We continue the example from Lemma 4.1 in Lemma 4.2. 
Lemma 4.2 Let $\mathfrak{g}(\lambda)_{\mathbb{F}}=\mathfrak{s l}_{2}(\mathbb{F})$ and let $\lambda=m \lambda_{1}$ for some $m \in \mathbb{N}$.

(a) Then, $V(\lambda)_{\mathbb{F}}$ is an $\mathfrak{s l}_{2}(\mathbb{F})$-module of dimension $m+1$.

(b) The module $V(\lambda)_{\mathbb{F}}$ has a basis $\left\{v_{0}, v_{1}, \ldots, v_{m}\right\}$ such that the formulas describing the $\mathfrak{s l}_{2}(\mathbb{F})$-action are as in Lemma $4.1(\mathrm{~b})$.

(c) If $\operatorname{Char}(\mathbb{F})=0$ or $\operatorname{Char}(\mathbb{F})=p$ and $m$ is restricted, i.e. $0 \leq m<p$, then $V(\lambda)_{\mathbb{F}}$ is cyclic as an $\mathfrak{s l}_{2}(\mathbb{F})$-module.

Proof Parts (a) and (b) follow immediately from Lemma 4.1. Part (c) follows from the fact that under these conditions all coefficients in the action formulas of Lemma 4.1 are non-zero in $\mathbb{Z}$ and remain so on reduction modulo $p$.

Remark 4.4 If one constructs the module $V(\lambda)_{\mathbb{F}}$ and the $\mathfrak{s l}_{2}(\mathbb{F})$-action from the maximal lattice $A_{\max }$, then there is a basis $\left\{f_{0}, \ldots, f_{m}\right\}$ such that the formulas describing the $\mathfrak{s l}_{2}(\mathbb{F})$-action are as in Lemma 4.1(c).

Remark 4.5 Irreducible modules with non-restricted weights can be constructed from those with restricted weights as described in [44, 46].

The Chevalley groups For any $\alpha \in \Phi^{+}$, and $n \in \mathbb{N}$, the elements $x_{\alpha, a}=X_{\alpha}^{a} / a$ ! and $y_{\alpha, a}=Y_{\alpha}^{a} / a$ ! belong to $\mathfrak{U}_{\mathbb{Z}}$ and hence leave $V(\lambda)_{\mathbb{Z}}$ invariant under their action on the module $V(\lambda)_{\mathbb{C}}$. Hence they also induce endomorphisms of $V(\lambda)_{\mathbb{F}}=\mathbb{F} \otimes_{\mathbb{Z}} V(\lambda)_{\mathbb{Z}}$, via a representation that we shall call $\rho$. Note that for large $a$, these elements represent the null operator on $V(\lambda) \mathbb{C}$. Therefore, for any constant $t \in \mathbb{F}$, the element

$$
x_{\alpha}(t)=\sum_{a=0}^{\infty} t^{a} \rho\left(x_{\alpha, a}\right)
$$

is an endomorphism of $V(\lambda)_{\mathbb{F}}$. It is a purely formal fact that $x_{\alpha}(t)^{-1}=x_{\alpha}(-t)$. We now let

$$
\mathrm{M}_{\Lambda(\lambda)}(\mathbb{F})=\left\langle x_{\alpha}(t), y_{\alpha}(t) \mid \alpha \in \Phi^{+}, t \in \mathbb{F}\right\rangle \leq \operatorname{SL}\left(V(\lambda)_{\mathbb{F}}\right) \leq \operatorname{End}\left(V(\lambda)_{\mathbb{F}}\right) .
$$

The group operation is the multiplication of $\operatorname{End}\left(V(\lambda)_{\mathbb{F}}\right)$, i.e. composition of endomorphisms. This group is a Chevalley group of type $M$ over $\mathbb{F}$. Up to isomorphism it only depends on $\Lambda(\lambda)$, but not on the choice of $A$. We shall also use the notation $\mathrm{M}_{\lambda}(\mathbb{F})=\mathrm{M}_{\Lambda(\lambda)}(\mathbb{F})$.

The group $M_{\Lambda_{r}}(\mathbb{F})$ is called the adjoint group and denoted $M_{a d}(\mathbb{F})$. It can be obtained from the adjoint representation of $\mathfrak{g}_{\mathbb{C}}$, whose highest weight is the "highest root", denoted $\alpha^{*}$ (see column 3 in Table 3 ). The group $\mathrm{M}_{\Lambda_{w}}(\mathbb{F})$ is called the universal group and denoted $\overline{\mathrm{M}}(\mathbb{F})$. Given $\lambda$ we have $\Lambda_{r} \leq \Lambda(\lambda) \leq \Lambda_{w}$ and central surjective homomorphisms $\overline{\mathrm{M}}(\mathbb{F}) \rightarrow \mathrm{M}_{\Lambda(\lambda)}(\mathbb{F})$ and $\mathrm{M}_{\Lambda(\lambda)}(\mathbb{F}) \rightarrow \mathrm{M}_{\mathrm{ad}}(\mathbb{F})$. We also have $Z(\overline{\mathrm{M}}(\mathbb{F})) \cong \operatorname{Hom}\left(\Lambda_{w} / \Lambda_{r}, \mathbb{F}^{*}\right)[45, \S 3]$. In particular, we can always view $V(\lambda)_{\mathbb{F}}$ as a $\overline{\mathrm{M}}(\mathbb{F})$-module, regardless of the choice of $\lambda$.

For types $E_{8}, F_{4}$ and $G_{2}$ we have $\Lambda_{r}=\Lambda_{w}$ so that $\mathrm{M}_{\mathrm{ad}}(\mathbb{F})=\overline{\mathrm{M}}(\mathbb{F})$. Table 3 lists the possible Chevalley groups for the other spherical types, along with some weights giving rise to these groups. Most of Table 3 comes from [45, §3]. In columns 3, 4, 


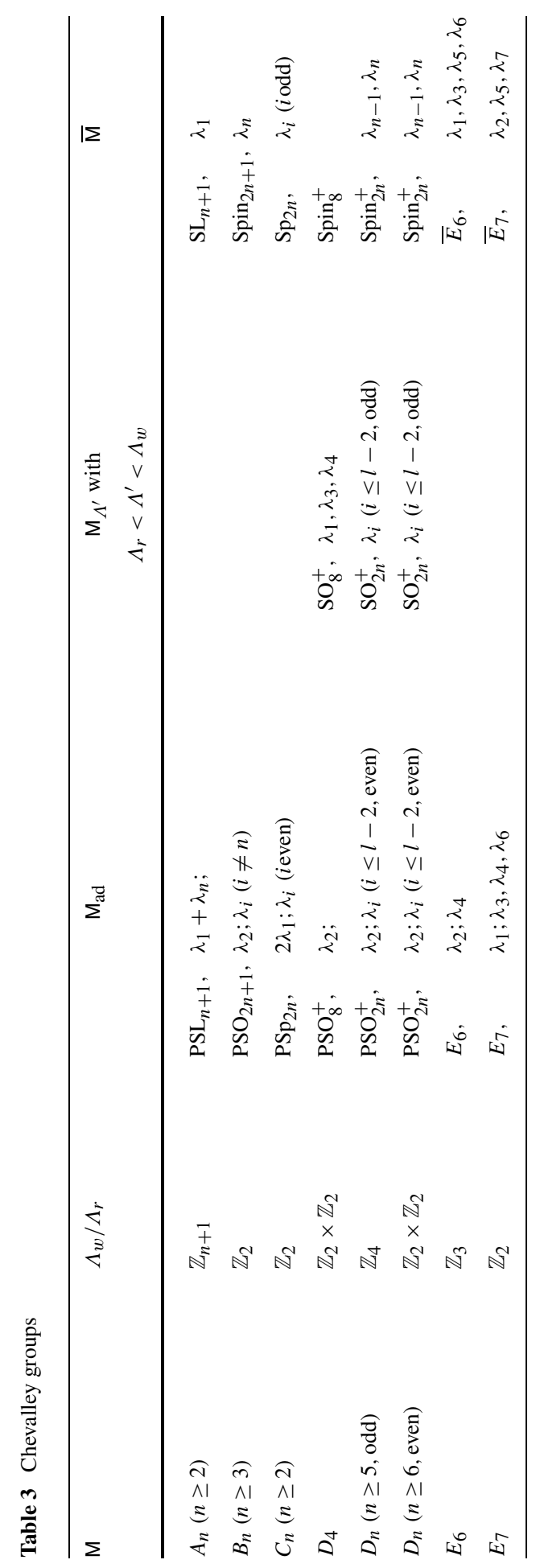


and 5, we list the group along with some weights $\lambda$ for which $M_{\Lambda(\lambda)}$ is the desired group. Dynkin diagram labelings are as in [8]. The first weight in column 3 is $\alpha^{*}$. The remaining weights can be found from Chaps. 8 and 13 in [15] with an easy calculation.

Remark 4.6 Steinberg's table [45, §3] also points out that $\mathrm{PSO}_{2 n+1} \cong \mathrm{SO}_{2 n+1}$. From Ree [35], we also have $B_{n, \text { ad }}=\mathrm{P} \Omega_{2 n+1}, \bar{B}_{n}=\Omega_{2 n+1}, D_{n, \text { ad }}=\mathrm{P} \Omega_{2 n}^{+}$, and $\bar{D}_{n}=\Omega_{2 n}^{+}$.

There does not seem to be a standard notation distinguishing the adjoint and universal Chevalley groups of types $E_{6}$ and $E_{7}$. We shall write $E_{n}(\mathbb{F})=E_{n \text {,ad }}(\mathbb{F})$ (and $\left.\bar{E}_{n}(\mathbb{F})=\bar{E}_{n}(\mathbb{F})\right)$.

From now on we shall write $G_{\mathrm{ad}}=\mathrm{M}_{\mathrm{ad}}(\mathbb{F}), G=\overline{\mathrm{M}}(\mathbb{F})$, and $G_{\lambda}(\mathbb{F})=\mathrm{M}_{\Lambda(\lambda)}(\mathbb{F})$.

The module $V(\lambda)_{\mathbb{F}}$ is in general not cyclic. In some cases it may depend on the choice of $A$. However, when for instance $\infty>\operatorname{dim}\left(V(\lambda)_{\mathbb{C}}\right)>\left|G_{\lambda}(\mathbb{F})\right|$, so that $V(\lambda)_{\mathbb{F}}$ has $\mathbb{F}$-dimension strictly greater than the size of the group algebra, the module cannot be cyclic.

Definition 4.2 We shall denote the $G_{\lambda}(\mathbb{F})$-module generated by $v^{+}$by $\mathbf{V}=V(\lambda)_{\mathbb{F}}^{0}$ and call this the Weyl module of highest weight $\lambda$.

For $V(\lambda)_{\mathbb{F}}$ itself we reserve the name weak Weyl module. Note that in some cases, such as when $\operatorname{Char}(\mathbb{F})=0$, these modules coincide.

We illustrate what may happen using the example from Lemma 4.2.

Lemma 4.3 Let $\mathrm{M}=A_{1}$. Write $\alpha=\alpha_{1}$ and let $\lambda=m \lambda_{1}$ for some $m \in \mathbb{N}$. Let $V=$ $V(\lambda)_{\mathbb{F}}$ be obtained from $V(\lambda)_{\mathbb{Z}}$. Then all of the following hold.

(a) The $G_{\lambda}(\mathbb{F})$-module $V$ has dimension $m+1$ and $\mathbb{F}$-basis $\left\{v_{0}, v_{1}, \ldots, v_{m}\right\}$, where $v_{i}=\left(Y_{\alpha}^{i} / i !\right) v^{+}$.

(b) If $\mathbb{F}$ has sufficiently many elements, then $V$ is cyclic and generated by $v^{+}$as $a$ $G_{\lambda}(\mathbb{F})$-module. This is the case if $\mathbb{F}$ is infinite or if $\mathbb{F}$ is finite, but $|\mathbb{F}| \geq m+1$.

(c) If $\operatorname{Char}(\mathbb{F})=0$, or $\operatorname{Char}(\mathbb{F})=p>0$ and $m$ is restricted, then $V$ is irreducible as a $G_{\lambda}(\mathbb{F})$-module.

Proof (a) Follows immediately from Lemma 4.2.

(b) Consider the element $y_{\alpha}(t)=\sum_{i=0}^{m}\left(\frac{Y_{\alpha}^{i}}{i !}\right) t^{i}$. We have $y_{\alpha}(t) v^{+}=\sum_{i=0}^{m} t^{i} v_{i}$. Therefore, if $\mathbb{F}$ contains distinct elements $t_{0}, \ldots, t_{m}$, then $\left(t_{j}^{i}\right)_{i, j=0}^{m}$ is a Vandermonde matrix. Hence the elements $y_{\alpha}\left(t_{0}\right) v^{+}, \ldots, y_{\alpha}\left(t_{m}\right) v^{+}$are linearly independent over $\mathbb{F}$.

(c) If $V^{\prime}$ is any submodule then it has a highest weight vector. Thus this highest weight vector is (a scalar multiple of) $v_{i}$ for some $i$. Note that, due to the fact that $m$ is restricted, all of the coefficients appearing in part (b) of Lemma 4.1 are non-zero over $\mathbb{F}$. Hence an argument similar to that of part (b) shows that $V^{\prime}$ also contains $v_{i+1}, \ldots, v_{m}$. Applying the argument of (b) to $x_{\alpha}(t) v_{m}$, we see that $V^{\prime}$ also contains $v_{0}, \ldots, v_{i-1}$ and so $V^{\prime}=V$. 
Remark 4.7 Suppose that we pick some finite field $\mathbb{F}_{p}, p$ prime and a weight $\lambda=m \lambda_{1}$ with $m>\left|\mathrm{SL}_{2}\left(\mathbb{F}_{p}\right)\right|$. Then, from Lemma 4.3 we see that while $V(\lambda) \mathbb{C}$ is irreducible for $\mathrm{SL}_{2}(\mathbb{C})$, the module $V(\lambda)_{\mathbb{F}_{p}}$ is not cyclic since its dimension exceeds that of the group algebra $\mathbb{F}_{p} \mathrm{SL}_{2}\left(\mathbb{F}_{p}\right)$. So in this case $\mathbf{V}=V(\lambda)_{\mathbb{F}_{p}}^{0}$ is a proper submodule of $V(\lambda)_{\mathbb{F}_{p}}$.

The $B N$-pair of $G_{\lambda}(\mathbb{F})$ To finish this subsection we identify some relevant subgroups of $G_{\lambda}(\mathbb{F})$, following [14].

The group

$$
G_{\alpha}=\left\langle x_{\alpha}(t), y_{\alpha}(t) \mid t \in \mathbb{F}\right\rangle
$$

is a Chevalley group over $\mathbb{F}$ with diagram $A_{1}$. By the above discussion therefore, $G_{\lambda}(\mathbb{F})$ is a quotient of $\mathrm{SL}_{2}(\mathbb{F})$, the universal group of type $A_{1}$, and it has the adjoint group $\mathrm{PSL}_{2}(\mathbb{F})$ as a quotient. More precisely, for each $\alpha$ we have a surjective homomorphism

$$
\begin{aligned}
\phi_{\alpha}: \mathrm{SL}_{2}(\mathbb{F}) & \rightarrow G_{\alpha}, \\
\left(\begin{array}{ll}
1 & t \\
0 & 1
\end{array}\right) & \mapsto x_{\alpha}(t), \\
\left(\begin{array}{ll}
1 & 0 \\
t & 1
\end{array}\right) & \mapsto y_{\alpha}(t) .
\end{aligned}
$$

The Steinberg presentation theorem [45] says that in case $G_{\lambda}(\mathbb{F})$ is universal, $G_{\lambda}(\mathbb{F})$ can be viewed as being generated abstractly by elements of the form $x_{\alpha}(t)$ and $y_{\alpha}(t),\left(\alpha \in \Phi^{+}, t \in \mathbb{F}\right)$ subject to the Chevalley commutator relations (see e.g. [14, Chap. 5]), and such that the above homomorphisms are in fact isomorphisms.

For each $\alpha \in \Phi^{+}$and $t \in \mathbb{F}$, let

$$
h_{\alpha}(t)=\phi_{\alpha}\left(\begin{array}{cc}
t & 0 \\
0 & t^{-1}
\end{array}\right), \quad n_{\alpha}(t)=\phi_{\alpha}\left(\begin{array}{cc}
0 & t \\
-t^{-1} & 0
\end{array}\right) \text {. }
$$

For each $\alpha \in \Phi^{+}$, let

$$
\begin{aligned}
U_{\alpha} & =\left\langle x_{\alpha}(t) \mid t \in \mathbb{F}\right\rangle, & & H_{\alpha}=\left\langle h_{\alpha}(t) \mid t \in \mathbb{F}\right\rangle, \\
U_{-\alpha} & =\left\langle y_{\alpha}(t) \mid t \in \mathbb{F}\right\rangle, & & N_{\alpha}=\left\langle n_{\alpha}(t) \mid t \in \mathbb{F}\right\rangle .
\end{aligned}
$$

We now have the following distinguished subgroups of $G_{\lambda}(\mathbb{F})$ :

$$
\begin{array}{lll}
U^{+}=\left\langle U_{\alpha} \mid \alpha \in \Phi^{+}\right\rangle, & H=\left\langle H_{\alpha} \mid \alpha \in \Phi^{+}\right\rangle, & B^{+}=U^{+} H, \\
U^{-}=\left\langle U_{\alpha} \mid \alpha \in \Phi^{-}\right\rangle, & N=\left\langle N_{\alpha} \mid \alpha \in \Phi^{+}\right\rangle, & B^{-}=U^{-} H .
\end{array}
$$

We also set $n_{i}=n_{\alpha_{i}}(1)$, for all $i \in I$.

Theorem 4.1 (See [14]) Let $B=B^{+}$. Then, the pair $(B, N)$ is a BN-pair of type $\mathrm{M}$ for $G_{\lambda}(\mathbb{F})$. More precisely, setting $W=N / H$ and $r_{i}=n_{i} H$, for $i \in I$, the pair $\left(W,\left\{r_{i}\right\}_{i \in I}\right)$ is a Coxeter system of type $\mathrm{M}$. 


\subsection{The Weyl embedding}

Continuing the notation from this section we now let

$$
\lambda=\lambda_{K}, \quad \mathbf{V}=V(\lambda)_{\mathbb{F}}^{0}, \quad G=\overline{\mathrm{M}}(\mathbb{F}) .
$$

Let $U=U^{+}, U^{-}, B=B^{+}, B^{-}, N$ and $H$ be defined as in Sect. 4.2, starting with the universal Chevalley group $G$. We assume that $\Delta$ is obtained from the $B N$-pair $(B, N)$ of $G$. We also assume that $\Gamma$ is the $K$-shadow space obtained from $\Delta$ as in Sect. 4.1

Theorem 4.2 The shadow space $\Gamma=(\mathcal{P}, \mathcal{L})$ has a full projective embedding into the Weyl module $\mathbf{V}$ for $G$ as follows

$$
\begin{aligned}
e_{W}: \mathcal{P} & \rightarrow \mathbb{P}(\mathbf{V}), \\
g p & \mapsto\left\langle g v^{+}\right\rangle,
\end{aligned}
$$

for any choice of a point $p$ of $\Gamma$.

Proof The proof is the same as the proof of Lemma 3.1 in [5], after noting that the weight $\lambda=\lambda_{K}$ is restricted for any characteristic, and $\mathbf{V}$ is cyclic by definition.

Definition 4.3 We shall call the map $e_{W}$ from Theorem 4.2 the Weyl embedding.

Remark 4.8 Clearly if we consider an arbitrary weight $\lambda=\sum_{i \in I} m_{i} \lambda_{i}$, where $m_{i} \in \mathbb{N}$, and we define the support of $\lambda$ to be the set $\operatorname{supp}(\lambda)=\left\{i \in I \mid m_{i}>0\right\}$, then $\mathbf{V}=V(\lambda)_{\mathbb{F}}^{0}$ affords some kind of embedding of the shadow space of type $I-\operatorname{supp}(\lambda)$. The arguments used in the proof of Theorem 4.2 show that the point-set is mapped into the set of 1-spaces of $\mathbf{V}$. The standard line of type $k$ is now mapped to an arc in the $\left(m_{k}+1\right)$-dimensional subspaces $\mathbf{V}_{k}=\left\langle g v^{+} \mid g \in P_{i}\right\rangle_{\mathbf{V}}$. Such modules are wellknown, so studying such embeddings is within reach and some of them might be interesting.

\section{The minimal polarized embedding obtained from the Weyl embedding}

In this section we show that the Weyl embedding is polarized and that its minimal polarized quotient is the unique irreducible module $L\left(\lambda_{K}\right)_{\mathbb{F}}$ of highest weight $\lambda_{K}$.

\subsection{Opposite structures, actions and representations}

The reader familiar with opposite structures can skip this subsection. In general, if $\mathbf{K}$ is a category, then the opposite category $\mathbf{K}^{\mathrm{opp}}$ is the category with the same objects as $\mathbf{K}$, but with arrows reversed. Thus the identity map id: $\mathbf{K} \rightarrow \mathbf{K}^{\mathrm{opp}}$ is a contravariant functor. For example, if $G$ is a group with operation $*$, then the opposite group $G^{\text {opp }}$ is the set $G$ equipped with the opposite operation $*^{\text {opp }}$ given by $x *^{\text {opp }} y=y * x$ for 
all $x, y \in G$. Note that if $G$ is commutative, then id: $G \rightarrow G^{\text {opp }}$ is an isomorphism. Similarly, if $R$ is a ring with multiplication $*$, then the opposite ring $R^{\text {opp }}$ is the additive group of $R$ equipped with the opposite multiplication $*$ opp .

Now let $R$ be a commutative ring with 1 . The definition of the opposite of an associative $R$-algebra $A$ now follows from the definitions above. If $\mathfrak{L}$ is a Lie algebra over $R$ with bracket $[\cdot, \cdot]$, then the opposite Lie algebra $\mathfrak{L}^{\text {opp }}$ is the $R$-module $\mathfrak{L}$ equipped with the opposite bracket given by $[x, y]^{\text {opp }}=[y, x]$. For an associative $R$-algebra $A$ with multiplication $*$, let $\mathfrak{L}(A)$ denote the Lie algebra on the $R$-module $A$ equipped with the bracket $[x, y]=x * y-y * x$. Then, we have $\mathfrak{L}\left(A^{\mathrm{opp}}\right)=\mathfrak{L}(A)^{\mathrm{opp}}$.

An isomorphism between a group, ring, algebra, or Lie algebra $X$, and its opposite $X^{\text {opp }}$ is called an anti-automorphism of $X$.

\subsection{The contravariant form on $V(\lambda)_{\mathbb{F}}$ and the irreducible quotient $L(\lambda)_{\mathbb{F}}$}

In this section we follow the approach sketched in [27, Sect. 3.8] and [52, 54]. We first define an automorphism $\tau$ of the vector space underlying $\mathfrak{g}_{\mathbb{C}}$ by setting, for each $\alpha \in \Phi$, (see e.g. [26]):

$$
X_{\alpha}^{\tau}=Y_{\alpha}, \quad H_{\alpha}^{\tau}=H_{\alpha}, \quad Y_{\alpha}^{\tau}=X_{\alpha}
$$

and extending $\mathbb{C}$-linearly. It follows from the isomorphism theorem for semi-simple complex Lie algebras [28, Chap. IV] that $\tau$ induces an anti-automorphism of $\mathfrak{g}_{\mathbb{C}}$. Naturally this extends to an isomorphism, also denoted $\tau$, between $\mathfrak{U}=\mathfrak{U}\left(\mathfrak{g}_{\mathbb{C}}\right)$ and $\mathfrak{U}\left(\mathfrak{g}_{\mathbb{C}}^{\text {opp }}\right)$. We can view $\mathfrak{U}\left(\mathfrak{g}_{\mathbb{C}}^{\text {opp }}\right)$ as $\mathfrak{U}^{\text {opp }}$ as follows.

First note that, as vector spaces, $\mathfrak{g}_{\mathbb{C}}=\mathfrak{g}_{\mathbb{C}}^{\text {opp }}$ and that the tensor algebra $T\left(\mathfrak{g}_{\mathbb{C}}\right)$ and its opposite $T^{\text {opp }}\left(\mathfrak{g}_{\mathbb{C}}^{\text {opp }}\right)$ are isomorphic via the map $r$, given on pure vectors by $x_{1} \otimes \cdots \otimes x_{n} \mapsto x_{n} \otimes \cdots \otimes x_{1}\left(n \in \mathbb{N}, x_{i} \in \mathfrak{g}_{\mathbb{C}}\right)$. Thus we can construct $\mathfrak{U}=T\left(\mathfrak{g}_{\mathbb{C}}\right) / \mathfrak{I}$ and $\mathfrak{U}\left(\mathfrak{g}_{\mathbb{C}}^{\text {opp }}\right)=T^{\text {opp }}\left(\mathfrak{g}_{\mathbb{C}}^{\text {opp }}\right) / \mathfrak{I}^{\text {opp }}$ (see e.g. [15]), where $\mathfrak{I}=\langle x \otimes y-y \otimes x-[x, y]|$ $\left.x, y \in \mathfrak{g}_{\mathbb{C}}\right\rangle$ and $\mathfrak{I}^{\text {opp }}=\left\langle x \otimes^{\text {opp }} y-y \otimes^{\text {opp }} x-[x, y]^{\text {opp }} \mid x, y \in \mathfrak{g}_{\mathbb{C}}^{\text {opp }}\right\rangle$ are two-sided ideals of the associative algebras. Now note that $\mathfrak{I}=\mathfrak{I}^{\text {opp }}$ as subspaces of the vector space $T\left(\mathfrak{g}_{\mathbb{C}}\right)=T^{\text {opp }}\left(\mathfrak{g}_{\mathbb{C}}^{\text {opp }}\right)$, so that as vector spaces $\mathfrak{U}=\mathfrak{U}\left(\mathfrak{g}_{\mathbb{C}}^{\text {opp }}\right)$. Hence $\mathfrak{U}\left(\mathfrak{g}_{\mathbb{C}}^{\text {opp }}\right)$ can alternatively be constructed by taking the opposite associative algebra of $\mathfrak{U}$ and taking its Lie algebra, or, equivalently, by taking the opposite Lie algebra structure of $\mathfrak{U}$. Thus, $\tau$ extends to a proper anti-automorphism of the universal enveloping algebra $\mathfrak{U}$ and its underlying associative algebra structure inherited from $T\left(\mathfrak{g}_{\mathbb{C}}\right)$. Since $\tau$ preserves $C$, it restricts to a proper anti-automorphism of the Kostant $\mathbb{Z}$ form $\mathfrak{U}_{\mathbb{Z}}$.

From $\tau$ one creates a symmetric bilinear form $\beta_{\mathbb{Z}}$ on $V(\lambda)_{\mathbb{Z}}$. Namely, one first defines a twisted $\mathfrak{g}_{\mathbb{C}}$-module ${ }^{\tau} V(\lambda)_{\mathbb{C}}$. The module ${ }^{\tau} V(\lambda)_{\mathbb{C}}$ is the dual vector space $V(\lambda)_{\mathbb{C}}^{*}$ with a twisted action defined as follows:

$$
(g \cdot f)(v)=f\left(g^{\tau}(v)\right) \quad \text { for all } g \in \mathfrak{g}_{\mathbb{C}}, f \in V(\lambda)_{\mathbb{C}}^{*}, v \in V(\lambda) \mathbb{C} .
$$

One verifies that ${ }^{\tau} V(\lambda) \mathbb{C}$ has highest weight vector $f^{+}$of weight $\lambda$, defined by

$$
f^{+}(v)=c \text { if } v \in c v^{+}+\bigoplus_{\mu \neq \lambda} V(\lambda)_{\mathbb{C}, \mu} .
$$


The map $\phi: V(\lambda)_{\mathbb{C}} \rightarrow{ }^{\tau} V(\lambda) \mathbb{C}$ given by $u v^{+} \mapsto u f^{+}(u \in \mathfrak{U})$ induces a unique

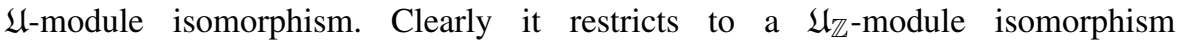
$\phi: \mathfrak{U}_{\mathbb{Z}} v^{+} \rightarrow \mathfrak{U}_{\mathbb{Z}} f^{+}$. We now define a bilinear form $\beta$ on $V(\lambda)_{\mathbb{C}}$ by setting

$$
\beta\left(v_{1}, v_{2}\right)=\phi\left(v_{1}\right)\left(v_{2}\right) \text { for all } v_{1}, v_{2} \in V(\lambda)_{\mathbb{C}}
$$

The form $\beta$ has the following properties (see $\S 3.8$ and $\S 2.4$ of [27]).

\section{Lemma 5.1}

(a) The bilinear form $\beta$ is contravariant. That is,

$$
\beta(g u, v)=\beta\left(u, g^{\tau} v\right) \quad \text { for all } g \in \mathfrak{U} \text {, and all } u, v \in V(\lambda)_{\mathbb{C}}
$$

(b) The form $\beta$ is symmetric and non-degenerate on $V(\lambda)_{\mathbb{C}}$.

(c) Weight spaces corresponding to distinct weights of $V(\lambda)_{\mathbb{C}}$ are orthogonal with respect to $\beta$.

(d) We have $\beta\left(v^{+}, v^{+}\right)=1$. Hence, it restricts to $\beta: A_{\min } \times A_{\min } \rightarrow \mathbb{Z}$. Mutatis mutandis, $(a),(b)$, and $(c)$ also hold for this restriction.

We note that in Lemma 5.1 part (c) is a consequence of (a).

Tensoring $\beta$ over $\mathbb{Z}$ with $\mathbb{F}$, we obtain a symmetric $\mathbb{F}$-bilinear contravariant form $\beta$ on $V(\lambda)_{\mathbb{F}}$.

\section{Corollary 5.1}

(a) The form $\beta$ induced on $V(\lambda)_{\mathbb{F}}$ is a symmetric $\mathbb{F}$-bilinear contravariant form such that $\beta\left(v^{+}, v^{+}\right)=1$.

(b) Reduced weight spaces of $V(\lambda)_{\mathbb{F}}$ corresponding to distinct weights of $V(\lambda)_{\mathbb{C}}$ are orthogonal with respect to $\beta$. Hence, weight spaces corresponding to distinct weights of $V(\lambda)_{\mathbb{F}}$ are orthogonal with respect to $\beta$.

Statements $(a)$ and $(b)$ also hold when $V(\lambda)_{\mathbb{F}}$ is replaced with the submodule $V(\lambda)_{\mathbb{F}}^{0}$ generated by $v^{+}$.

Example 5.1 Let $\mathfrak{g}_{\mathbb{C}}=\mathfrak{s l}_{2}(\mathbb{C})$ and $\lambda=m \lambda_{1}$, for some $m \in \mathbb{N}$. The minimal admissible lattice $A_{\min }=V(\lambda)_{\mathbb{Z}}$ is the $\mathbb{Z}$-span of the basis $\mathcal{V}=\left\{v_{0}, \ldots, v_{m}\right\}$ from Lemma 4.1 part (b). We now construct ${ }^{\tau} V(\lambda)_{\mathbb{C}}$. Let $\left\{f_{0}, f_{1}, \ldots, f_{m}\right\}$ be the basis of $V(\lambda)_{\mathbb{C}}^{*}$ dual to $\mathcal{V}$, i.e. such that $f_{i}\left(v_{j}\right)=\delta_{i, j}$ for $0 \leq i, j \leq m$. Using the formulas from Lemma 4.1 part (b), together with the fact that $\tau$ fixes $H_{\alpha_{1}}$ and interchanges $X_{\alpha_{1}}$ and $Y_{\alpha_{1}}$ we find that the action of $\mathfrak{s l}_{2}(\mathbb{C})$ on ${ }^{\tau} V(\lambda) \mathbb{C}$ is given by the formulas in part (c) of that lemma. The isomorphism $\phi: V(\lambda)_{\mathbb{C}} \rightarrow{ }^{\tau} V(\lambda)_{\mathbb{C}}$ is given by $v_{i} \mapsto\left(\begin{array}{c}m \\ i\end{array}\right) f_{i}$ for all $i=0,1, \ldots, m$. Hence, with respect to the basis $\mathcal{V}$, $\beta$ is given by the diagonal matrix with entry $\left(\begin{array}{c}m \\ i\end{array}\right)$ in the $i$ th row and column. We have $\Lambda(\lambda)=\left\{(m-2 i) \lambda_{1} \mid i=0,1, \ldots, m\right\}$ with $A_{\min ,(m-2 i) \lambda_{1}}=\mathbb{Z} v_{i}$, for each $i$. Lemma 5.1 and Corollary 5.1 are easily verified in this case. Also, the maximal lattice in $V(\lambda)_{\mathbb{C}}$ corresponding to $A_{\min }$ is $A_{\max }=\left\{f \in V(\lambda)_{\mathbb{C}} \mid \beta(f, a) \in \mathbb{Z} \forall a \in A_{\min }\right\}$, which is the $\mathbb{Z}$-span of $\left\{v_{i} /\left(\begin{array}{c}m \\ i\end{array}\right) \mid i=0, \ldots, m\right\}$. 
We now extend the preceding results to the Chevalley groups.

Proposition 5.1 The map $\tau$ induces an anti-automorphism of $G_{\lambda}(\mathbb{F})$ that satisfies

$$
x_{\alpha}(t)^{\tau}=x_{-\alpha}(t) \quad \text { for all } \alpha \in \Phi, t \in \mathbb{F} .
$$

Before we prove Proposition 5.1, we introduce some notation connecting the various module structures involved with the corresponding representations.

Let $\rho: \mathfrak{g}_{\mathbb{C}} \rightarrow \mathfrak{g l}\left(V(\lambda)_{\mathbb{C}}\right)$ denote the representation corresponding to the leftmodule structure: $\rho(x)(v)=x \star v$. Let $\langle\cdot, \cdot\rangle: V(\lambda)_{\mathbb{C}}^{*} \times V(\lambda)_{\mathbb{C}} \rightarrow \mathbb{C}$ be the standard pairing given by $\langle f, v\rangle=f(v)$. One verifies that the $\mathbb{C}$-linear map $\rho^{\dagger}: \mathfrak{g}_{\mathbb{C}}^{\text {opp }} \rightarrow$ $\mathfrak{g l}\left(V(\lambda)_{\mathbb{C}}^{*}\right)$ denoted $\rho^{\dagger}(x)(f)=x \star^{\dagger} f$ and given by $\left\langle x \star^{\dagger} f, v\right\rangle=\langle f, x \star v\rangle$, for all $x \in \mathfrak{g}_{\mathbb{C}}^{\text {opp }}, f \in V(\lambda)_{\mathbb{C}}^{*}$, and $v \in V(\lambda)_{\mathbb{C}}$ is a homomorphism. The composition $\rho^{\tau}=$ $\rho^{\dagger} \circ \tau$ is the representation $\rho^{\tau}: \mathfrak{g}_{\mathbb{C}} \rightarrow \mathfrak{g l}\left(V(\lambda)_{\mathbb{C}}^{*}\right)$ that turns $V(\lambda)_{\mathbb{C}}^{*}$ into the $\mathfrak{g}_{\mathbb{C}}$-module ${ }^{\tau} V(\lambda) \mathbb{C}$. We denote the action $x \star^{\tau} f=\rho^{\tau}(x)(f)$. It satisfies $x \star^{\tau} f=x^{\tau} \star^{\dagger} f$.

We then have natural extensions to the universal enveloping algebras $\mathfrak{U}, \mathfrak{U}^{\text {opp }}$ that we denote $\rho, \rho^{\tau}$, and $\rho^{\dagger}$ as well. The isomorphism $\phi$ sends $x \star v^{+} \mapsto x \star^{\tau} f^{+}$, for all $x \in \mathfrak{U}$. Setting $V(\lambda)_{\mathbb{Z}}=\mathfrak{U}_{\mathbb{Z}} \star v^{+}$and $V(\lambda)_{\mathbb{Z}}^{*}=\mathfrak{U}_{\mathbb{Z}}^{\text {opp }} \star^{\dagger} f^{+}=\mathfrak{U}_{\mathbb{Z}} \star^{\tau} f^{+}$, we see that $\phi$ restricts to $\phi: V(\lambda)_{\mathbb{Z}} \rightarrow V(\lambda)_{\mathbb{Z}}^{*}$. Since we also have $\left\langle f^{+}, v^{+}\right\rangle=1$, the pairing restricts to $\langle\cdot, \cdot\rangle: V(\lambda)_{\mathbb{Z}}^{*} \times V(\lambda)_{\mathbb{Z}} \rightarrow \mathbb{Z}$. Therefore we have restrictions $\rho: \mathfrak{U}_{\mathbb{Z}} \rightarrow \mathfrak{g l}\left(V(\lambda)_{\mathbb{Z}}\right)$, $\rho^{\dagger}: \mathfrak{U}_{\mathbb{Z}}^{\text {opp }} \rightarrow \mathfrak{g l}\left(V(\lambda)_{\mathbb{Z}}^{*}\right)$, and $\rho^{\tau}: \mathfrak{U}_{\mathbb{Z}} \rightarrow \mathfrak{g l}\left(V(\lambda)_{\mathbb{Z}}^{*}\right)$, such that $\phi$ induces an isomorphism between $\rho$ and $\rho^{\tau}$, and, for every $f \in V(\lambda)_{\mathbb{Z}}^{*}, x \in \mathfrak{U}_{\mathbb{Z}}$, and $v \in V(\lambda)_{\mathbb{Z}}$, we have $\left\langle x \star^{\dagger} f, v\right\rangle=\langle f, x \star v\rangle$. Combining all this we find that, for $v_{1}, v_{2} \in V(\lambda)_{\mathbb{Z}}$, we have $\beta\left(x \star v_{1}, v_{2}\right)=\left\langle\phi\left(x \star v_{1}\right), v_{2}\right\rangle=\left\langle x \star^{\tau} \phi\left(v_{1}\right), v_{2}\right\rangle=\left\langle x^{\tau} \star^{\dagger} \phi\left(v_{1}\right), v_{2}\right\rangle=$ $\left\langle\phi\left(v_{1}\right), x^{\tau} \star v_{2}\right\rangle=\beta\left(v_{1}, x^{\tau} \star v_{2}\right)$, i.e. $\beta$ is $\tau$-contravariant.

As stated in Corollary 5.1, when we pass to the field $\mathbb{F}$, these properties are preserved. First tensor $\tau$ to get an isomorphism $\tau_{\mathbb{F}}: \mathfrak{U}_{\mathbb{F}} \rightarrow \mathfrak{U}_{\mathbb{F}}^{\text {opp }}$. Next, we tensor the representations $\rho, \rho^{\dagger}$ and $\rho^{\tau}$. For example, we define $\rho_{\mathbb{F}}: \mathfrak{U}_{\mathbb{F}} \rightarrow \mathfrak{g l}\left(V(\lambda)_{\mathbb{F}}\right)$ by $c \otimes x \mapsto c \otimes \rho(x)$ and with corresponding action $(c \otimes x) \star(d \otimes v)=c d \otimes x \star v$. The others are defined similarly. Note that $\rho_{\mathbb{F}}$ also defines a homomorphism between the underlying associative algebras. One verifies that $\rho_{\mathbb{F}}^{\tau}=\rho_{\mathbb{F}}^{\dagger} \circ \tau_{\mathbb{F}}$. Ten-

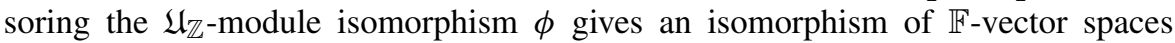
$\phi_{\mathbb{F}}: V(\lambda)_{\mathbb{F}} \rightarrow V(\lambda)_{\mathbb{F}}^{*}$. Conjugation by $\phi_{\mathbb{F}}^{-1}$ gives an isomorphism of associative algebras $\phi_{\mathbb{F}}^{*}: \operatorname{End}\left(V(\lambda)_{\mathbb{F}}\right) \rightarrow \operatorname{End}\left(V(\lambda)_{\mathbb{F}}^{*}\right)$, which also induces an isomorphism between the corresponding Lie algebras $\phi^{*}: \mathfrak{g l}\left(V(\lambda)_{\mathbb{F}}\right) \rightarrow \mathfrak{g l}\left(V(\lambda)_{\mathbb{F}}^{*}\right)$. Thus we have the following commutative diagram of associative algebra morphisms.

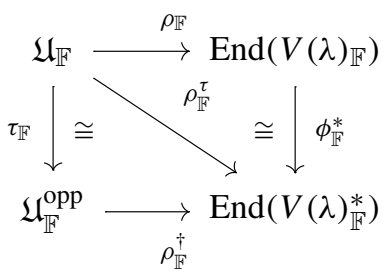


Proof of Proposition 5.1 We shall construct the Chevalley group $G_{\lambda}(\mathbb{F})$ as well as the group $G_{\lambda}^{*}(\mathbb{F})$ for $\mathfrak{g}_{\mathbb{C}}^{\text {opp }}$. We then show that $G_{\lambda}^{*}(\mathbb{F})$ is naturally isomorphic to the opposite group $G_{\lambda}(\mathbb{F})^{\text {opp }}$ and that $\tau$ induces an isomorphism $G_{\lambda}(\mathbb{F})^{*} \cong G_{\lambda}(\mathbb{F})^{\text {opp }}$.

The group $G_{\lambda}(\mathbb{F})$ is generated as a subgroup of the multiplicative group of $\operatorname{End}\left(V(\lambda)_{\mathbb{F}}\right)$ ) by images under $\rho_{\mathbb{F}}$ of elements of the form $\overline{x_{\alpha}}(t)=\sum_{a=0}^{\infty} t^{a} \otimes x_{\alpha, a}$. Thus we have $\rho_{\mathbb{F}}\left(\overline{x_{\alpha}}(t)\right)=\sum_{a=0}^{\infty} t^{a} \otimes \rho\left(x_{\alpha, a}\right)$ and the action on $V(\lambda)_{\mathbb{F}}=\mathbb{F} \otimes_{\mathbb{Z}}$ $V(\lambda)_{\mathbb{Z}}$ is therefore given by $x_{\alpha}(t) \star(1 \otimes v)=\sum_{a=0}^{\infty} t^{a} \otimes\left(x_{\alpha, a} \star v\right)$, for any $\alpha \in \Phi$, $t \in \mathbb{F}$, and $v \in V(\lambda)_{\mathbb{Z}}$.

If $\mathbb{V}$ is the category of finite dimensional $\mathbb{F}$-vector spaces and linear transformations and $\mathrm{D}: \mathbb{V} \rightarrow \mathbb{V}$ denotes the duality functor, sending each $V \in \mathbb{V}$ to its dual $V^{*} \in$ $\mathbb{V}$ and each linear transformation $E$ to $E^{*}: f \mapsto f \circ E$, then $\mathrm{D}$ is contravariant and $\mathrm{D}^{\mathrm{opp}}: \mathbb{V} \rightarrow \mathbb{V}^{\text {opp }}$ is covariant. Now view $\operatorname{End}\left(V(\lambda)_{\mathbb{F}}\right)$ as the subcategory with single object $V(\lambda)_{\mathbb{F}}$ in which the morphisms are those of $\mathbb{V}$ having $V(\lambda)_{\mathbb{F}}$ as starting point and end point. Since $V(\lambda)_{\mathbb{F}}$ is finite dimensional, we have $V(\lambda)_{\mathbb{F}}^{* *}=V(\lambda)_{\mathbb{F}}$ and it follows that $\mathrm{D}^{\mathrm{opp}}$ restricts to an isomorphism between $\operatorname{End}\left(V(\lambda)_{\mathbb{F}}\right)$ and $\operatorname{End}\left(V(\lambda)_{\mathbb{F}}^{*}\right)^{\text {opp }}$.

Composing Dopp with $\rho_{\mathbb{F}}: \mathfrak{U}_{\mathbb{F}} \rightarrow \operatorname{End}\left(V(\lambda)_{\mathbb{F}}\right)$ we get a dual representation $\rho_{\mathbb{F}}^{* o p p}=$ $\mathrm{D}^{\text {opp }} \rho_{\mathbb{F}}: \mathfrak{U}_{\mathbb{F}} \rightarrow \operatorname{End}\left(V(\lambda)_{\mathbb{F}}^{*}\right)^{\text {opp }}$. Taking opposites in the domain and codomain of $\rho_{\mathbb{F}}^{* \text { opp }}$, one verifies that we recover the representation $\rho_{\mathbb{F}}^{\dagger}: \mathfrak{U}_{\mathbb{F}}^{\text {opp }} \rightarrow \operatorname{End}\left(V(\lambda)_{\mathbb{F}}^{*}\right)$ introduced above. Thus all triangles in Diagram (2), which includes Diagram (1), commute.

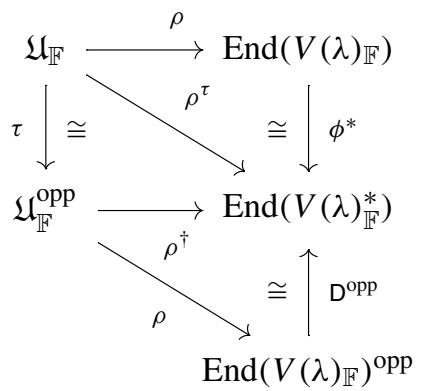

Let $G_{\lambda}^{*}(\mathbb{F})$ be the Chevalley group for $\mathfrak{g}_{\mathbb{F}}^{\text {opp }}$ obtained using $\rho_{\mathbb{F}}^{\dagger}$. Clearly D: $\operatorname{End}\left(V(\lambda)_{\mathbb{F}}\right)$ $\rightarrow \operatorname{End}\left(V(\lambda)_{\mathbb{F}}^{*}\right)$ satisfies $\rho_{\mathbb{F}}\left(\overline{x_{\alpha}}(t)\right) \mapsto \rho_{\mathbb{F}}^{\dagger}\left(\overline{x_{\alpha}}(t)\right)$, for all $\alpha \in \Phi$ and $t \in \mathbb{F}$, and, being bijective and contravariant, it restricts to an anti-isomorphism between $G_{\lambda}(\mathbb{F})$ and $G_{\lambda}^{*}(\mathbb{F})$.

From the commutativity of Diagram (2), we deduce that $\tau$ induces an isomorphism $\phi^{*}: G_{\lambda}(\mathbb{F}) \rightarrow G_{\lambda}(\mathbb{F})^{*}$ satisfying $\rho\left(\overline{x_{\alpha}}(t)\right) \mapsto \rho^{\dagger}\left(\overline{x_{-\alpha}}(t)\right)$ as well as an antiautomorphism: $\mathrm{D}^{\mathrm{opp}} \circ \phi^{*}: G_{\lambda}(\mathbb{F}) \rightarrow G_{\lambda}(\mathbb{F})^{\text {opp }}$ which satisfies $\rho\left(\overline{x_{\alpha}}(t)\right) \mapsto \rho\left(\overline{x_{-\alpha}}(t)\right)$. This is the sought anti-automorphism.

We shall denote the anti-automorphism of $G_{\lambda}(\mathbb{F})$ induced by $\tau$ also by $\tau$.

It is now straightforward to verify the following.

Lemma 5.2 For any $g \in G_{\lambda}(\mathbb{F})$ and $u, v \in V(\lambda)_{\mathbb{F}}$ we have

$$
\beta(g u, v)=\beta\left(u, g^{\tau} v\right) .
$$


Recall from Sect. 4.2 that $n_{i}=n_{\alpha_{i}}(1)$, for $i \in I$.

Lemma 5.3 The subgroup $N_{0}=\left\langle n_{i} \mid i \in I\right\rangle \leq N$ of the universal Chevalley group $G$ is an isometry group for the form $\beta$.

Proof First we note that for each $\alpha \in \Phi$ and $t \in \mathbb{F}$ we have $x_{\alpha}(t)^{\tau}=x_{-\alpha}(t)$ and it follows that $n_{\alpha}(t)^{\tau}=\left(x_{\alpha}(t) x_{-\alpha}\left(-t^{-1}\right) x_{\alpha}(t)\right)^{\tau}=n_{\alpha}\left(-t^{-1}\right)$. In particular, $n_{\alpha}(1)^{\tau}=$ $n_{\alpha}(1)^{-1}$. Recall also that $\tau$ is an anti-automorphism, meaning that $(g h)^{\tau}=h^{\tau} g^{\tau}$ for any $g, h \in G_{\lambda}(\mathbb{F})$. It follows that if $n=n_{i_{1}} \cdots n_{i_{l}}$, then $n^{\tau}=n^{-1}$. Hence, for $u, v \in V(\lambda)_{\mathbb{F}}$, we have $\beta(n u, n v)=\beta\left(u, n^{\tau} n v\right)=\beta(u, v)$.

It follows from Lemma 5.3 that weight vectors in one $N_{0}$-orbit have the same length with respect to $\beta$. It should be pointed out that in Lemma $5.3 N_{0}$ cannot be replaced by $N$ in general.

Our motivation for introducing the contravariant form $\beta$ is that it gives a connection between the Weyl module $\mathbf{V}=V(\lambda)_{\mathbb{F}}^{0}$ and the unique irreducible module $L(\lambda)_{\mathbb{F}}$ of highest weight $\lambda$ (see [27]).

Proposition 5.2 (cf. $[27, \S 3.8]$ ) The Weyl module $\mathbf{V}=V(\lambda)_{\mathbb{F}}^{0}$ has a unique maximal $G_{\lambda}(\mathbb{F})$-submodule and this submodule equals the radical of $\beta$ in $\mathbf{V}$. As a consequence, $\beta$ induces a non-degenerate contravariant form on the simple quotient $L(\lambda)_{\mathbb{F}}$.

Proof The proof is the same as that of Proposition 3.8 of [27] after noting that $\mathbf{V}=$ $V(\lambda)_{\mathbb{F}}^{0}$ is cyclic.

\subsection{The Weyl embedding is polarized}

Recall that $G_{\lambda}(\mathbb{F})$ is a central quotient of the universal Chevalley group $G$. Hence any $G_{\lambda}(\mathbb{F})$-module is automatically a $G$-module.

Proposition 5.3 The Weyl embedding $e_{W}$ of $\Gamma$ into $\mathbb{P}(\mathbf{V})$ is polarized. More precisely, for each point $p$ of $\Gamma$ there is a dual point $p^{*}$ of $\Gamma$ such that

$$
e_{W}(p)^{\perp}=\left\langle H\left(p^{*}\right)\right\rangle_{e_{W}} .
$$

Proof Let $\Sigma$ be the apartment of $\Delta$ corresponding to $N$ and let $c^{+}$be the chamber corresponding to $B$. Let $p$ be the point of $\Gamma$ on $c^{+}$.

Recall that $\mathbf{V}=V(\lambda)_{\mathbb{F}}^{0}$. For any weight $\mu \in \Lambda(\lambda)$, let $\mathbf{V}_{\mu}$ denote its reduced weight space as defined in Definition 4.1. Recall that $e_{W}(p)$ is the subspace $\mathbf{V}_{\lambda}$ of $\mathbf{V}$ spanned by $1 \otimes v^{+}$, where $v^{+}$is the highest weight vector. Also note that for any point $q$ of $\Sigma$ we have $q=w p$ for some $w \in W-\{1\}$ so that $e_{W}(q)=w \mathbf{V}_{\lambda}=\mathbf{V}_{\mu}$. Now if $p \neq q$, then $\operatorname{since} \operatorname{dim}\left(\mathbf{V}_{\lambda}\right)=1$, we must have $\mu=w \lambda \neq \lambda$. Thus, $\beta\left(e_{W}(p), e_{W}(q)\right)=0$ for all points $q$ on $\Sigma$ different from $p$.

Note that the chamber $c^{-}$opposite to $c^{+}$in $\Sigma$ corresponds to $B^{-}=w_{0} B^{+} w_{0}$, where $w_{0}$ is the longest word in the Coxeter system $\left(W,\left\{r_{i}\right\}_{i \in I}\right)$. Now let $p^{*}$ be the dual point of $\Gamma^{*}$ on $c^{-}$and let $H\left(p^{*}\right)$ be the hyperplane of $\Gamma$ consisting of points 
not opposite to $p^{*}$ in $\Delta$. Then for every point $q^{\prime} \in H\left(p^{*}\right)$ there is an apartment $\Sigma^{\prime}$ on $q^{\prime}$ and $c^{-}$. The group $B^{-}$stabilizes $p^{*}$ while being regular on such apartments. So there is some $b \in B^{-}$with $b \Sigma=\Sigma^{\prime}$ and there is some point $q$ on $\Sigma$ such that $b q=q^{\prime}$. Since $q^{\prime}$ was not opposite to $p^{*}, q \neq p$.

Turning back to the embedding we note that by contravariance and since $b^{\tau} \in$ $B^{+}=B$, we have $\beta\left(e_{W}(p), e_{W}\left(q^{\prime}\right)\right)=\beta\left(e_{W}(p), b e_{W}(q)\right)=\beta\left(b^{\tau} e_{W}(p), e_{W}(q)\right)=$ $\beta\left(e_{W}(p), e_{W}(q)\right)=0$. Thus the hyperplane $e_{W}(p)^{\perp}=\operatorname{ker}\left(\beta\left(v^{+},-\right)\right)$contains $\left\langle H\left(p^{*}\right)\right\rangle_{e_{W}}$. Since $H\left(p^{*}\right)$ is a maximal subspace of $\Gamma$ and using Lemma 1.2, we find

$$
e_{W}(p)^{\perp}=\left\langle H\left(p^{*}\right)\right\rangle_{e_{W}} .
$$

Part (b) of Lemma 1.2 moreover tells us that the hyperplane $H\left(p^{*}\right)$ is induced by $\mathbf{V}$. Since $G$ and hence also $G_{\lambda}(\mathbb{F})$ are transitive on dual points, and since $\beta$ is contravariant, the same holds for all other dual points of $\Gamma^{*}$. Thus $\mathbf{V}$ is polarized.

Corollary 5.2 Let $e_{W}$ be the Weyl embedding of $\Gamma$ into the Weyl module $\mathbf{V}$ and let $\mathcal{R}$ be the polar radical of $e_{W}$. Then the codomain of the minimal polarized embedding with respect to $e_{W}$ is the unique irreducible $G_{\lambda}(\mathbb{F})$-module $L(\lambda)_{\mathbb{F}}$ of highest weight $\lambda$.

Proof From Proposition 5.3 it follows that

$$
\mathcal{R}=\bigcap_{p^{*} \in \Gamma^{*}}\left\langle H\left(p^{*}\right)\right\rangle_{e_{W}}=\bigcap_{p \in \Gamma} e_{W}(p)^{\perp}=\operatorname{Rad}(\beta) .
$$

Therefore the codomain of the minimal polarized embedding with respect to $e_{W}$ is $\mathbf{V} / \mathcal{R}=V(\lambda)_{\mathbb{F}}^{0} / \operatorname{Rad}(\beta)$, which by Proposition 5.2 is the unique irreducible $G_{\lambda}(\mathbb{F})$ module of highest weight $\lambda$.

Theorem 1.2 now follows from Proposition 5.3 and Corollary 5.2.

\section{Minuscule weight geometries}

Let $\lambda=\lambda_{k}$ be a fundamental dominant weight that is minuscule. This means by definition that the weight lattice of $V(\lambda)_{\mathbb{C}}$ equals the orbit of $\lambda$ under the action of the Weyl group $W$. In particular, all weight spaces have dimension 1 . Recall that $\lambda_{k}$ is a minuscule weight for the diagram $\mathrm{M}$ if $\mathrm{M}_{k}$ is one of the following: $A_{n, k}$ (any $k$ ), $B_{n, n}$ $(n \geq 2), C_{n, 1}(n \geq 3), D_{n, 1}, D_{n, n-1}, D_{n, n}(n \geq 4), E_{6,1}$ and $E_{6,6}$, or $E_{7,7}$. For names and dimensions of these embeddings see Table 4 .

Call $e=e_{W}$ the embedding of $\Gamma=\Delta_{k}$ into $\mathbf{V}=V(\lambda)_{\mathbb{F}}^{0}$. The weight spaces of $V$ are precisely the images of the point set of the apartment $\Sigma=W P_{I-\{k\}}$ of $\Gamma$ corresponding to $W$, and in almost all cases these points generate $\Gamma$ (see $[5,20]$ for a precise statement). By Theorem 1.2 the embedding $e$ into $\mathbf{V}$ is polarized. Moreover, $V(\lambda)_{\mathbb{F}}=\mathbf{V}=L(\lambda)_{\mathbb{F}}$ since if $V(\lambda)_{\mathbb{F}}$ had any proper submodule, it would be the direct sum of its weight spaces. But the weight spaces of $V(\lambda) \mathbb{F}$ are all of dimension 1 and form a single orbit under $W$. Hence, no proper submodule exists. In view of 
Table 4 Minuscule weight embeddings

\begin{tabular}{lllll}
\hline $\mathrm{M}$ & $G$ & $k$ & $\mathbf{V}$ & $\operatorname{dim}(\mathbf{V})$ \\
\hline$A_{n}$ & $\mathrm{SL}_{n+1}(\mathbb{F})$ & $k$ & Grassmann & $\left(\begin{array}{c}n+1 \\
k\end{array}\right)$ \\
$B_{n}$ & $\operatorname{Spin}_{2 n+1}(\mathbb{F})$ & $n$ & Spin & $2^{n}$ \\
$C_{n}$ & $\operatorname{Sp}_{2 n}(\mathbb{F})$ & 1 & Natural & $2 n$ \\
$D_{n}$ & $\operatorname{Spin}_{2 n}^{+}(\mathbb{F})$ & 1 & Natural & $2 n$ \\
& & $n, n-1$ & Half-spin & $2^{n-1}$ \\
$E_{6}$ & $\bar{E}_{6}(\mathbb{F})$ & 1,6 & $L\left(\lambda_{k}\right)$ & 27 \\
$E_{7}$ & $\bar{E}_{7}(\mathbb{F})$ & 7 & $L\left(\lambda_{7}\right)$ & 56 \\
\hline
\end{tabular}

Lemma 5.3 it also implies that $\mathbf{V}$ has a basis of weight-vectors that is orthonormal with respect to $\beta$. Therefore, $\beta$ has trivial radical, which by Theorem 1.2 again implies that $V(\lambda)_{\mathbb{F}}=\mathbf{V}$ is irreducible.

We finish this section with a brief remark on generating singular hyperplanes. Let $p$ be a point of $\Sigma$ opposite some dual point $p^{*}$ also on $\Sigma$. We now see that the hyperplane $\left\langle H\left(p^{*}\right)\right\rangle_{e}$ is exactly the hyperplane of $\mathbf{V}$ spanned by the set $\{e(q) \mid p \neq$ $q$ a point of $\Sigma$ \}. It is proved in Blok [3] that often the hyperplane $H\left(p^{*}\right)$ itself is generated, as a subspace of $\Gamma$ by the set of points of $\Sigma$ different from $p$; this is the case for instance if the diagram $\mathrm{M}$ is one of $A_{n}, D_{n}, E_{6}$ or $E_{7}$.

In Table 4 we list the Weyl embeddings of the minuscule weight geometries. Here $\Gamma$ is the $k$-shadow space of a building associated with the universal Chevalley group $G=\overline{\mathrm{M}}(\mathbb{F})$ and $V=V\left(\lambda_{k}\right)_{\mathbb{F}}=\mathbf{V}=L\left(\lambda_{k}\right)_{\mathbb{F}}$.

\section{Grassmannians}

As a preliminary to Sects. 8, 9 and 10, we collect some information on tensor products and exterior powers of modules for the Lie algebra $\mathfrak{g}=\mathfrak{g}(\lambda)_{\mathbb{F}}$ and its associated Chevalley group $G_{\lambda}(\mathbb{F})$. In particular, we shall study the form $\beta$ and the automorphism $\tau$.

It is well known (see e.g. [15]) and easy to check that, whenever $V_{1}, \ldots, V_{l}$ are $\mathfrak{g}$-modules, then so is $V_{1} \otimes \cdots \otimes V_{l}$ under the action given on pure vectors by

$$
g \cdot \bigotimes_{i=1}^{l} v_{i}=\sum_{i=1}^{l} v_{1} \otimes \cdots \otimes v_{i-1} \otimes g v_{i} \otimes v_{i+1} \otimes \cdots \otimes v_{l},
$$

for all $g \in \mathfrak{g}$ and $v_{i} \in V_{i}$ for all $i=1, \ldots, l$. Similarly, if $V$ is a $\mathfrak{g}$-module, then so is $\wedge^{k} V$ under the action given on pure vectors by

$$
g \cdot \bigwedge_{i=1}^{k} v_{i}=\sum_{i=1}^{k} v_{1} \wedge \cdots \wedge v_{i-1} \wedge g v_{i} \wedge v_{i+1} \wedge \cdots \wedge v_{k},
$$

for all $g \in \mathfrak{g}$ and $v_{i} \in V$ for all $i=1, \ldots, k$. As for the action of $G_{\lambda}(\mathbb{F})$, it is well known and it follows easily from (3) and the definition of $G_{\lambda}(\mathbb{F})$, that whenever 
$V_{1}, \ldots, V_{l}$ are $G_{\lambda}(\mathbb{F})$-modules, then so is $V_{1} \otimes \cdots \otimes V_{l}$ under the action given on pure vectors by

$$
g \cdot \bigotimes_{i=1}^{l} v_{i}=g v_{1} \otimes \cdots \otimes g v_{i} \otimes \cdots \otimes g v_{l},
$$

for all $g \in G_{\lambda}(\mathbb{F})$ and $v_{i} \in V_{i}$ for all $i=1, \ldots, l$. Similarly, if $V$ is a $G_{\lambda}(\mathbb{F})$-module, then so is $\bigwedge^{k} V$ under the action given on pure vectors by

$$
g \cdot \bigwedge_{i=1}^{k} v_{i}=g v_{1} \wedge \cdots \wedge g v_{i} \wedge \cdots \wedge g v_{k},
$$

for all $g \in G_{\lambda}(\mathbb{F})$ and $v_{i} \in V$ for all $i=1, \ldots, k$.

Next, we describe how a covariant or contravariant form on a collection of modules for $\mathfrak{g}$ or $G_{\lambda}(\mathbb{F})$ induces a similar form on their tensor product or exterior powers.

Lemma 7.1 Let $V_{1}, \ldots, V_{l}$ be finite dimensional $\mathbb{F}$-vector spaces, let $\sigma$ be an automorphism of $\mathbb{F}$ of order at most 2 and let $\zeta_{i}$ be a $\sigma$-sesquilinear form on $V_{i}$. Then

(a) there is a unique $\sigma$-sesquilinear form $\zeta^{\otimes}$ on $V_{1} \otimes \cdots \otimes V_{l}$ given by

$$
\zeta^{\otimes}\left(u_{1} \otimes \cdots \otimes u_{l}, v_{1} \otimes \cdots \otimes v_{l}\right)=\prod_{i=1}^{l} \zeta_{i}\left(u_{i}, v_{i}\right)
$$

(b) if each $\zeta_{i}$ is non-degenerate, so is $\zeta^{\otimes}$;

(c) if each $\zeta_{i}$ is symmetric bilinear, so is $\zeta^{\otimes}$;

(d) if each $\zeta_{i}$ is skew-symmetric, then $\zeta^{\otimes}$ is skew symmetric if $l$ is odd and symmetric otherwise;

(e) if each $V_{i}$ is a module for $\mathfrak{g}$ and $\zeta_{i}$ is $\tau$-contravariant, then so is $\zeta^{\otimes}$;

(f) if each $V_{i}$ is a module for $G_{\lambda}(\mathbb{F})$ and $\zeta_{i}$ is $\tau$-contravariant, then so is $\zeta^{\otimes}$;

(g) if each $V_{i}$ is a module for $G_{\lambda}(\mathbb{F})$ and $G_{\lambda}(\mathbb{F})$ preserves $\zeta_{i}$, then $G_{\lambda}(\mathbb{F})$ preserves $\zeta^{\otimes}$.

Proof (a) For each $i$ we have a $\sigma$-semilinear map $\phi_{i}: V_{i} \rightarrow V_{i}^{*}$ so that $\zeta_{i}(u, v)=$ $\left\langle\phi_{i}(u), v\right\rangle$, where $\langle f, v\rangle=f(v)$ is the standard pairing $V_{i}^{*} \times V_{i} \rightarrow \mathbb{F}$. Note that $\phi^{\otimes}=$ $\phi_{1} \otimes \cdots \otimes \phi_{l}: V_{1} \otimes \cdots \otimes V_{l} \rightarrow V_{1}^{*} \otimes \cdots \otimes V_{l}^{*}$ is again a $\sigma$-semilinear map. We can compose this map with the standard pairing $V_{1}^{*} \otimes \cdots \otimes V_{l}^{*} \times V_{1} \otimes \cdots \otimes V_{l} \rightarrow \mathbb{F}$ given by $\left\langle f_{1} \otimes \cdots \otimes f_{l}, v_{1} \otimes \cdots \otimes v_{l}\right\rangle=\prod_{i=1}^{l} f_{i}\left(v_{i}\right)$ to get the form $\zeta^{\otimes}$. It is immediate from this construction that $\zeta^{\otimes}$ is $\sigma$-sesquilinear. (b) The standard pairing is non-degenerate and in this case $\phi_{i}$ and $\phi^{\otimes}$ are isomorphisms. (c) and (d) are trivial observations. (e), (f), and (g) are easily seen to follow from (3) and (5).

Lemma 7.2 Let $V$ be a finite dimensional $\mathbb{F}$-vector space, let $\sigma$ be an automorphism of $\mathbb{F}$ of order at most 2 and let $\zeta$ be a $\sigma$-sesquilinear form on $V$. Let $k \in \mathbb{N}$ with $1 \leq k \leq \operatorname{dim}(V)$. Then

(a) there is a unique $\sigma$-sesquilinear form $\zeta^{\wedge}$ on $\wedge^{k} V$ given by

$$
\zeta^{\wedge}\left(u_{1} \wedge \cdots \wedge u_{k}, v_{1} \wedge \cdots \wedge v_{k}\right)=\operatorname{det}\left(\zeta\left(u_{i}, v_{j}\right)\right)
$$

(b) if $\zeta$ is non-degenerate, so is $\zeta^{\wedge}$;

(c) if $\zeta$ is symmetric bilinear, so is $\zeta^{\wedge}$; 
(d) if $\zeta$ is skew-symmetric, then $\zeta^{\wedge}$ is skew symmetric if $k$ is odd and symmetric otherwise;

(e) if $V$ is a module for $\mathfrak{g}$ and $\zeta$ is $\tau$-contravariant, then so is $\zeta^{\wedge}$;

(f) if $V$ is a module for $G_{\lambda}(\mathbb{F})$ and $\zeta$ is $\tau$-contravariant, then so is $\zeta^{\wedge}$;

$(\mathrm{g})$ if $V$ is a module for $G_{\lambda}(\mathbb{F})$ and $G_{\lambda}(\mathbb{F})$ preserves $\zeta$, then $G_{\lambda}(\mathbb{F})$ preserves $\zeta^{\wedge}$.

Proof (a) There is a $\sigma$-semilinear map $\phi: V \rightarrow V^{*}$ so that $\zeta(u, v)=\langle\phi(u), v\rangle$, where $\langle f, v\rangle=f(v)$ is the standard pairing $V^{*} \times V \rightarrow \mathbb{F}$. Note that $\phi^{\wedge}=\phi \wedge$ $\cdots \wedge \phi: \wedge^{k} V \rightarrow \wedge^{k} V^{*}$ is again a $\sigma$-semilinear map. We can compose this $\sigma$-semilinear map with the standard pairing $\wedge^{k} V^{*} \times \wedge^{k} V \rightarrow \mathbb{F}$ given by $\left\langle f_{1} \wedge \cdots \wedge\right.$ $\left.f_{l}, v_{1} \wedge \cdots \wedge v_{l}\right\rangle=\operatorname{det}\left(f_{i}\left(v_{j}\right)\right)$ to get the form $\zeta^{\wedge}$. Noting that $\operatorname{det}\left(\zeta\left(u_{i}, v_{j}\right)\right)=$ $\sum_{\rho \in \operatorname{Sym}(k)} \operatorname{sign}(\rho) \prod_{i=1}^{k} \zeta\left(u_{i}, v_{\rho(i)}\right)$ we see that $\zeta^{\wedge}$ is $\sigma$-sesquilinear because $\zeta^{\otimes}$ as defined in Lemma 7.1, is $\sigma$-sesquilinear. (b) The standard pairing is non-degenerate and in this case $\phi$ and $\phi^{\wedge}$ are isomorphisms. (c) This is because $\operatorname{det}\left(f_{i}\left(v_{j}\right)\right)=$ $\operatorname{det}\left(f_{j}\left(v_{i}\right)\right)$. (d) Same as in Lemma 7.1(e), (f) and (g). These follow from (4) and (6) together with the definition of the determinant as in (a).

Thus, we see that $\beta^{\wedge}$ and $\beta^{\otimes}$ are symmetric bilinear $\tau$-contravariant forms. Orthogonality of distinct weight spaces follows from contravariance as in Lemma 5.1.

\section{The projective Grassmannians}

Let $\Delta$ be the building of type $A_{n}$ over the field $\mathbb{F}$. The universal Chevalley group is $G=\mathrm{SL}(V)$, where $V$ is a vector space of dimension $n+1$ over $\mathbb{F}$. Picking an ordered basis $\mathcal{A}=\left\{a_{1}, \ldots, a_{n+1}\right\}$ for $V$, we identify $G$ with $\mathrm{SL}_{n+1}(\mathbb{F})$. A BN-pair for $G$ is given by letting $B$ be the upper triangular matrix group and $N$ the monomial matrix group. Then $H=B \cap N$ is the diagonal matrix group and the Weyl group $W=N / H \cong \operatorname{Sym}(n+1)$ in its action on the 1 -spaces spanned by the standard basis elements.

For each integer $k$ with $1 \leq k \leq n+1$, the exterior power $\bigwedge^{k} V$ is clearly a module for $G$ under the action $g\left(v_{1} \wedge \cdots \wedge v_{k}\right)=g v_{1} \wedge \cdots \wedge g v_{k}$. For any non-empty subset $J \subseteq I$, let $a_{J}=\bigwedge_{j \in J} a_{j}$, where the $a_{j}$ appear with increasing subscripts. Recall that $\mathcal{A}^{k}=\left\{a_{J}|J \subseteq\{1,2, \ldots, n+1\}| J \mid,=k\right\}$ is a basis for $\bigwedge^{k} V$. One verifies that $v^{+}=a_{\{1,2, \ldots, k\}}$ is a vector of weight $\lambda_{k}$ that is stabilized by the subgroup $U^{+}$of unipotent upper triangular matrices (see e.g. [15, Chap. 13]). Thus $v^{+}$is a vector of highest weight $\lambda_{k}$. It is easy to see that $\lambda_{k}$ is minuscule: the collection of 1 -spaces spanned by elements from $\mathcal{A}^{k}$ forms a single orbit under $W=\operatorname{Sym}(n+1)$. Thus, $\bigwedge^{k} V$ is the irreducible Weyl module $V\left(\lambda_{k}\right)_{\mathbb{F}}=\mathbf{V}=L\left(\lambda_{k}\right)_{\mathbb{F}}$.

The standard parabolic subgroup $P_{I-\{k\}}$ is precisely the stabilizer of the $k$-space $p=\left\langle a_{1}, \ldots, a_{k}\right\rangle_{V}$, which is a $k$-object of $\Delta$. The $k$-shadow space $\Gamma$ of $\Delta$ is the geometry whose points are the $k$-objects and where each line is the collection of points incident to some $\{k-1, k+1\}$-flag. 
In accordance with Theorem 4.2, the Weyl embedding of $\Gamma$ into $\wedge^{k} V$ is given by

$$
\begin{aligned}
\mathcal{P} & \rightarrow \mathbb{P}\left(\bigwedge^{k} V\right) \\
p & \mapsto \bigwedge^{k} p
\end{aligned}
$$

where $\wedge^{k} p=\left\langle p_{1} \wedge \cdots \wedge p_{k}\right\rangle$ for some basis $p_{1}, \ldots, p_{k}$ of $p$. It is often called the Grassmann embedding. This is well-defined since if $g \in \mathrm{SL}(V)$ induces a change of basis for $p$ we have $g\left(p_{1} \wedge \cdots \wedge p_{k}\right)=d p_{1} \wedge \cdots \wedge p_{k}$, where $d$ is the determinant of the restriction of $g$ to $p$.

Next, we identify the form $\beta$ on the module $\mathbf{V}$. Since $\lambda_{k}$ is minuscule, Sect. 6 tells us that $\mathcal{A}^{k}$ forms an orthonormal basis for $\wedge^{k} V$ with respect to $\beta$. Thus, if $\beta_{1}$ is the form on $V\left(\lambda_{1}\right)_{\mathbb{F}}$, then $\beta=\beta_{1}^{\wedge}$, as described in Lemma 7.2.

Let us also identify $\tau$. The anti-involution $\tau$ of $G$ as described in Sect. 5 satisfies $x_{\alpha}(t)^{\tau}=x_{-\alpha}(t)$ for any $t \in \mathbb{F}$ and $\alpha \in \Phi$. In the present $A_{n}$ case, the root system is $\Phi=\left\{\alpha_{i, j} \mid i, j \in I, i \neq j\right\}$ where $\alpha_{i, j}=-\alpha_{j, i}$ with respect to the fundamental system $\Pi=\left\{\alpha_{i, i+1} \mid i=1, \ldots, n\right\}$. With respect to the BN-pair chosen above we have $x_{\alpha_{i, j}}(t)=I_{n+1}+t E_{i, j}$, where $E_{i, j}$ is the elementary matrix whose entries $e_{k, l}$ satisfy $e_{k, l}=\delta_{i k} \delta_{j l}$. Thus, $\tau$ is simply the transposition map.

\section{Polar Grassmannians}

In this subsection $\Gamma$ is a polar $k$-Grassmannian of a building $\Delta$ of type $\mathrm{M}_{n}$ over $\mathbb{F}$, where $\mathrm{M}_{n, k}(\mathbb{F})$ is as listed in Table 5. The building $\Delta$ is constructed from a nondegenerate reflexive sesquilinear or quadratic form $\zeta$ of Witt index $n$ on a vector space $V$ of dimension $m$ over the field $\mathbb{F}$. The type of $\zeta$ is given in the table and $m$ is the subscript of the group, which is the full linear isometry group of $\zeta$. In case $\zeta$ is $\sigma$-hermitian, we restrict to the case where $\sigma \in \operatorname{Aut}(\mathbb{F})$ has order $2, \mathbb{F}$ is a quadratic extension over the fixed field $\mathbb{F}^{\sigma}=\left\{x \in \mathbb{F} \mid x^{\sigma}=x\right\}$, and the norm $N_{\sigma}: \mathbb{F} \rightarrow \mathbb{F}^{\sigma}$ is surjective.

We first present a way to see that the Grassmann embeddings for these polar Grassmannians are polarized. Then we shall analyze $\beta$ and $\tau$ for the untwisted cases $\left(B_{n}\right.$, $C_{n}$, and $\left.D_{n}\right)$.

The points and lines of $\Gamma$ are also points and lines of the projective $k$ Grassmannian $\bar{\Gamma}$ of type $A_{m-1, k}(\mathbb{F})$ associated with $V$ (see Sect. 8). The Grassmann embedding $\overline{e_{\mathrm{gr}}}$ of $\bar{\Gamma}$ restricts to a full projective embedding $e_{\mathrm{gr}}$ of $\Gamma$ into some subspace $V_{\mathrm{gr}}$ of the exterior power $\bigwedge^{k} V$. This is called the Grassmann embedding of $\Gamma$.

Proposition 9.1 Let $\Gamma$ be a polar k-Grassmannian as in Table 5. Then the Grassmann embedding of $\Gamma$ is polarized.

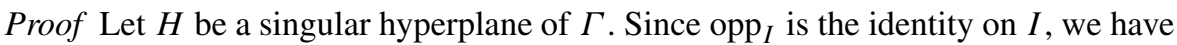
$\Gamma=\Gamma^{*}$ and so $H=H\left(p^{*}\right)$ consists of all points $q$ of $\Gamma$ not opposite to some point 
Table 5 Polar Grassmannians

\begin{tabular}{lllll}
\hline $\mathrm{M}_{n, k}(\mathbb{F})$ & $\zeta$ & Group & $n$ & $k$ \\
\hline$B_{n, k}(\mathbb{F})$ & Parabolic orthogonal & $\operatorname{Spin}_{2 n+1}(\mathbb{F})$ & $\geq 2$ & $1 \leq k<n$ \\
$C_{n, k}(\mathbb{F})$ & Symplectic & $\operatorname{Sp}_{2 n}(\mathbb{F})$ & $\geq 2$ & $1 \leq k \leq n$ \\
$D_{n, k}(\mathbb{F})$ & Hyperbolic orthogonal & $\Omega_{2 n}^{+}(\mathbb{F})$ & $\geq 3$ & $1 \leq k \leq n-2$ \\
${ }^{2} A_{2 n, k}(\mathbb{F})$ & $\sigma$-hermitian & $U_{2 n+1}(\mathbb{F})$ & $\geq 2$ & $1 \leq k<n$ \\
${ }^{2} A_{2 n-1, k}(\mathbb{F})$ & $\sigma$-hermitian & $U_{2 n}(\mathbb{F})$ & $\geq 2$ & $1 \leq k<n$ \\
${ }^{2} D_{n+1, k}(\mathbb{F})$ & Elliptic orthogonal & $\mathrm{SO}_{2 n+2}^{-}(\mathbb{F})$ & $\geq 2$ & $1 \leq k<n$ \\
\hline
\end{tabular}

$p^{*}$, which also belongs to $\Gamma$. Viewing points of $\Gamma$ as $k$-spaces in $V$, this means that $H$ consists of all points $q$ of $\Gamma$ such that $q \cap\left(p^{*}\right)^{\perp} \neq 0$. Here $\perp$ denotes orthogonality with respect to $\zeta$.

Keeping in mind that $\zeta$ is non-degenerate we find that $\bar{p}^{*}=\left(p^{*}\right)^{\perp}$ is an $(m-k)$ space of $V$, that is a dual point in $(\bar{\Gamma})^{*}$. Let $\bar{H}=H\left(\bar{p}^{*}\right)$ be the singular hyperplane of $\bar{\Gamma}$ defined by $\bar{p}^{*}$. Then $\bar{H}$ consists of all $k$-spaces $\bar{q}$ of $V$ with $\bar{q} \cap \bar{p}^{*} \neq 0$. Thus, $\langle H\rangle_{e_{\mathrm{gr}}} \leq\langle\bar{H}\rangle_{\overline{e_{\mathrm{gr}}}}$. We claim that in fact

$$
\langle H\rangle_{e_{\mathrm{gr}}}=V_{\mathrm{gr}} \cap\langle\bar{H}\rangle_{\overline{e_{\mathrm{gr}}}} .
$$

Since the Grassmann embedding $\overline{e_{\mathrm{gr}}}$ of $\bar{\Gamma}$ is polarized, $\langle\bar{H}\rangle_{\overline{e_{\mathrm{gr}}}}$ is a hyperplane of $\bigwedge^{k} V$ that induces $\bar{H}$. Since $\zeta$ is non-degenerate, there is a point $p$ in $\Gamma$ opposite to $p^{*}$, that is, not contained in $\left(p^{*}\right)^{\perp}=\bar{p}^{*}$. Thus we find that the codomain $V_{\mathrm{gr}}$ of $\Gamma$ under $e_{\mathrm{gr}}$ is not entirely contained in the hyperplane $\langle\bar{H}\rangle_{\overline{\mathrm{egr}}}$. Hence $\langle H\rangle_{e_{\mathrm{gr}}}$ is contained in the hyperplane $V_{\mathrm{gr}} \cap\langle\bar{H}\rangle_{\overline{e_{\mathrm{gr}}}}$ of $V_{\mathrm{gr}}$. By Proposition 1.1 and Lemma 1.2, $H$, is induced by $V_{\mathrm{gr}}$. That is, $e_{\mathrm{gr}}$ is polarized.

Recall from Lemma 7.2 that $\zeta$ induces a form $\zeta^{\wedge}$ on $\wedge^{k} V$ and hence on $V_{\mathrm{gr}}$ as follows. Namely, for $u_{1}, \ldots, u_{k}, v_{1}, \ldots, v_{k} \in V$, let

$$
\zeta^{\wedge}\left(u_{1} \wedge \cdots \wedge u_{k}, v_{1} \wedge \cdots \wedge v_{k}\right)=\operatorname{det}\left(\zeta\left(u_{i}, v_{j}\right)\right) .
$$

Let $\perp^{\wedge}$ denote the orthogonality relation on $\bigwedge^{k} V$ with respect to $\zeta^{\wedge}$. For any subspace $U \leq \wedge^{k} V$, let $\operatorname{Rad}\left(U, \zeta^{\wedge}\right)=U^{\perp^{\wedge}} \cap U$. By Lemma 7.2, since $G$ preserves $\zeta$, it also preserves $\zeta^{\wedge}$ and hence also its radical $\operatorname{Rad}\left(V_{\mathrm{gr}}, \zeta^{\wedge}\right)$.

Lemma 9.1 Let $\Gamma$ be a polar k-Grassmannian as in Table 5 .

(a) For any (dual) point $p^{*} \in \Gamma^{*}=\Gamma$ we have $\left\langle H\left(p^{*}\right)\right\rangle_{e_{\mathrm{gr}}}=e_{\mathrm{gr}}\left(p^{*}\right)^{\perp^{\wedge}}$;

(b) as a consequence $\mathcal{R}_{e_{\mathrm{gr}}}=\operatorname{Rad}\left(V_{\mathrm{gr}}, \zeta^{\wedge}\right)$.

Proof (a) First note that, by Lemma 7.2, $\zeta^{\wedge}$ is non-degenerate sesquilinear on $\bigwedge^{k} V$, so that $e_{\mathrm{gr}}\left(p^{*}\right)^{\perp^{\wedge}}$ is a proper hyperplane of $\wedge^{k} V$. On the other hand, by Proposition 9.1, we know that $\left\langle H\left(p^{*}\right)\right\rangle_{e_{\mathrm{gr}}}$ is a proper hyperplane of $V_{\mathrm{gr}}$. Thus, it suffices to prove that $\left\langle H\left(p^{*}\right)\right\rangle_{e_{\mathrm{gr}}} \subseteq e_{\mathrm{gr}}\left(p^{*}\right)^{\perp^{\wedge}}$. 
Let $u=u_{1} \wedge \cdots \wedge u_{k}$ represent $p^{*}$ and let $v=v_{1} \wedge \cdots \wedge v_{k}$ represent some point $q$ of $\Gamma$. Now $\zeta^{\wedge}(u, v)=0$ if and only if the columns of the matrix $\left(\zeta\left(u_{i}, v_{j}\right)\right)$ are linearly dependent, which happens if and only if $q \cap\left(p^{*}\right)^{\perp} \neq 0$ in $V$ and the latter is equivalent to saying that $q \in H\left(p^{*}\right)$. In particular, $\left\langle H\left(p^{*}\right)\right\rangle_{e_{\mathrm{gr}}} \subseteq e_{\mathrm{gr}}\left(p^{*}\right)^{\perp^{\wedge}}$.

For the untwisted cases, we choose $\zeta$ and the basis $\mathcal{A}$ for $V$ in the following way.

$$
\begin{aligned}
& \mathrm{M}_{n}: \quad B_{n}, \quad C_{n}, \quad D_{n}, \\
& \zeta:\left(\begin{array}{ccc}
2 & 0 & 0 \\
0 & O_{n} & I_{n} \\
0 & I_{n} & O_{n}
\end{array}\right), \quad\left(\begin{array}{cc}
O_{n} & I_{n} \\
-I_{n} & O_{n}
\end{array}\right), \quad\left(\begin{array}{cc}
O_{n} & I_{n} \\
I_{n} & O_{n}
\end{array}\right), \\
& \mathcal{A}: \quad\left\{a_{0}, \ldots, a_{2 n}\right\}, \quad\left\{a_{1}, \ldots, a_{2 n}\right\}, \quad\left\{a_{1}, \ldots, a_{2 n}\right\} .
\end{aligned}
$$

Lemma 9.2 Let $\Gamma$ be a polar k-Grassmannian as in Table 5, where $\mathrm{M}_{n}$ is untwisted, that is, it is one of $B_{n}, C_{n}$ or $D_{n}$. Then, the Grassmann embedding is the Weyl embedding.

Proof The Grassmann embedding is the restriction of the Grassmann embedding for the projective $k$-Grassmannian into $\bigwedge^{k} V$. The codomain $V_{\mathrm{gr}}$ is by definition the subspace of $\wedge^{k} V$ spanned by the images of the points of $\Gamma$. Since we have $G \leq$ $\operatorname{SL}(V)$, the space $V_{\mathrm{gr}}$ is naturally a $G$-module. Transitivity of $G$ on the point set of $\Gamma$ shows that $V_{\mathrm{gr}}$ is the $G$-submodule of $\bigwedge^{k} V$ generated by $e_{\mathrm{gr}}(p)$ for any given point $p$ of $\Gamma$.

More precisely, under the Grassmann embedding the point $p=\left\langle a_{1}, \ldots, a_{k}\right\rangle$ of $\Gamma$ is sent to the 1 -space of $\bigwedge^{k} V$ spanned by $a_{1, \ldots, k}$. Since $G \leq \operatorname{SL}(V)$ in all cases, we see that the Grassmann embedding is given by

$$
\begin{aligned}
e_{\mathrm{gr}}: \mathcal{P} & \rightarrow \mathbb{P}\left(\bigwedge^{k} V\right), \\
g p & \mapsto\left\langle g a_{1, \ldots, k}\right\rangle, \quad \text { for any } g \in G .
\end{aligned}
$$

We now consider the Weyl embedding. First let $\mathbb{F}=\mathbb{C}$. From [15] we see that $v^{+}=$ $a_{1, \ldots, k} \in \bigwedge^{k} V$ is a vector of highest weight $\lambda_{k}$. Now $V(\lambda)_{\mathbb{C}}=V(\lambda)_{\mathbb{C}}^{0}$ is the $G_{\mathbb{C}^{-}}$ module generated by $v^{+}$.

Passing to an arbitrary field $\mathbb{F}$, we see that $V(\lambda)_{\mathbb{F}}$ is also a $G_{\mathbb{F}}$-submodule of $\wedge^{k} V$ (where $V$ is now an $\mathbb{F}$-vector space) that contains the 1-dimensional highest weight space $V(\lambda)_{\mathbb{F}, \lambda}=\mathbb{F} v^{+}$. The Weyl module $\mathbf{V}$ is by definition the $G_{\mathbb{F}}$-submodule of $V(\lambda) \mathbb{F}$ generated by $v^{+}$.

Remark 9.1 Note that we now have two proofs of the fact that the Grassmann embedding of a polar $k$-Grassmannian associated with a polar space of type $B_{n}, C_{n}$, or $D_{n}$ 
is polarized. Namely, Proposition 9.1 gives a direct geometric proof, whereas another proof comes from combining Lemma 9.2 and Proposition 5.3.

We now describe $\tau$ in terms of the description, given in [15], of the Lie algebra $\mathfrak{g}_{\mathbb{C}}$ and $G$ in its action on the natural module. From the description of the root spaces in the Lie algebras of type $C_{n}$, and $D_{n}$ in loc. cit., we see that $\tau$ is given by the transpose map on $\mathfrak{g}_{\mathbb{C}}$, and hence also on $G$. In the $B_{n}$ case $\tau$ is given by $g \mapsto h^{-1} g^{t} h$, where $t$ denotes transpose and $h$ is the diagonal matrix $\operatorname{diag}\{2,1, \ldots, 1\}$. This formula, which is initially computed for $\operatorname{Char}(\mathbb{F})=0$, remains valid when $\operatorname{Char}(\mathbb{F}) \neq 2$.

Remark 9.2 The forms $\zeta^{\wedge}$ and $\beta$ are not equal, but it follows from Lemmas 9.1 and 9.2 that for each $p \in \Gamma$ there is a $p^{*} \in \Gamma^{*}=\Gamma$ such that $e_{\mathrm{gr}}\left(p^{*}\right)^{\perp^{\wedge}}=e_{\mathrm{gr}}(p)^{\perp^{\beta}}$, where $\perp^{\beta}$ denotes orthogonality with respect to the contravariant form $\beta$. In particular this means that $\operatorname{Rad}\left(V_{\mathrm{gr}}, \beta\right)=\operatorname{Rad}\left(V_{\mathrm{gr}}, \zeta^{\wedge}\right)$.

\section{Projective flag-Grassmannians}

We continue the setup from Sect. 8 except that now $\Gamma$ is a $K$-shadow space of $\Delta$, for some arbitrary non-empty subset $K \subseteq I=\{1,2, \ldots, n\}$. By Theorem 4.2, $\Gamma$ embeds into the Weyl module $\mathbf{V}=V\left(\lambda_{K}\right)_{\mathbb{F}}^{0}$ for $\mathfrak{g}_{\mathbb{F}}=\mathfrak{s l}_{n+1}(\mathbb{F})$, which is the submodule of $V\left(\lambda_{K}\right)_{\mathbb{F}}$ generated by the highest weight vector $v^{+}$. In this section, we construct the Weyl module from the natural $\mathfrak{s l}_{n+1}(\mathbb{F})$-module $V$, of highest weight $\lambda_{1}$. We give two constructions and study the form $\beta$.

Write $\lambda=\lambda_{K}$. Our construction of $V(\lambda)_{\mathbb{F}}$ follows [23]. Starting from a $\mathbb{D}$-module $V$, where $\mathbb{D}$ is some integral domain, we shall give two descriptions of a space $V_{\mathbb{D}}^{\lambda}$, with the property that $V_{\mathbb{F}}^{\lambda}=V(\lambda)_{\mathbb{F}}$, when $\mathbb{F}$ is a field (see Theorem 10.1). To unburden our notation we shall drop the $\mathbb{D}$ unless strictly needed. The condition that $\mathbb{D}$ be an integral domain is not necessary in all that follows (see loc. cit.), but it is all we need.

These constructions are valid for any weight $\lambda=\sum_{k \in K} l_{k} \lambda_{k}$, where $\lambda_{k}$ is the $k$ th fundamental dominant weight. To this end we identify $\lambda$ with the Young diagram that has $l_{k}$ columns of length $k$. That is, the partition corresponding to the (rows of the) transpose of the diagram $\lambda$ is $\mu=\left(k^{l_{k}}\right)_{k \in K}$, where $k$ runs through $K$ in decreasing order; we sometimes write $\mu=\left(\mu_{1}, \ldots, \mu_{l}\right)$, where $l=\sum_{k \in K} l_{k}$. In the constructions below we shall in fact assume $K \subseteq[n+1]=\{1,2, \ldots, n+1\}$ so as to also include those modules $V^{\lambda}$ involving determinantal representations.

A universal description of $V^{\lambda}$ Assume that $\lambda$ has $d$ boxes. Let $V^{\times \lambda}$ be a cartesian product of $d$ copies of $V$ indexed by the boxes of $\lambda$. Consider maps $\phi: V^{\times \lambda} \rightarrow W$, where $W$ is some $\mathbb{D}$-module, with the following properties:

(i) $\phi$ is $\mathbb{D}$-linear in each argument;

(ii) $\phi$ is alternating in each column of $\lambda$;

(iii) for any $x \in V^{\times \lambda}, \phi(x)=\sum \phi(y)$, where $y$ runs through all vectors obtained from $x$ by an exchange between two given columns of $\lambda$ with a given set of boxes in the right chosen column. 
$x=\left(v_{1}, v_{2}, v_{3}, v_{4}, v_{5}\right) \leftrightarrow$

\begin{tabular}{|l|l|}
\hline$v_{3}$ & $v_{5}$ \\
\hline$v_{2}$ & $v_{4}$ \\
\hline$v_{1}$ & \multicolumn{1}{|c}{} \\
\cline { 1 - 2 } &
\end{tabular}

\begin{tabular}{|l|l|}
\hline$v_{5}$ & $v_{3}$ \\
\hline$v_{2}$ & $v_{1}$ \\
\hline$v_{4}$ & \multicolumn{1}{|l}{} \\
\cline { 1 - 1 }
\end{tabular}

Fig. 1 An exchange between two sets of two boxes in columns 1 and 2

If $x$ and $y$ are vectors indexed by the boxes of the same Young diagram $\lambda$, then an exchange between $x$ and $y$ is determined by choosing, in $\lambda$, two distinct columns $i$ and $j$ along with a set of $s \geq 1$ boxes in both columns; $y$ is now obtained from $x$ by interchanging the coordinates of $x$ corresponding to the $s$ boxes in columns $i$ and $j$ of $\lambda$, while preserving their relative order within those columns. For an illustration, see Fig. 1. For example, taking $\lambda=\lambda_{3}+\lambda_{2}$ and using rules (i), (ii), and (iii) with $i=1, j=2$, and $s=2$, for $v_{1}, \ldots, v_{5} \in V$ and $\alpha \in \mathbb{D}$, we require that $\phi\left(\alpha v_{1}, v_{2}, v_{3}, v_{4}, v_{5}\right)=\alpha \phi\left(v_{1}, v_{4}, v_{5}, v_{2}, v_{3}\right)-\alpha \phi\left(v_{2}, v_{4}, v_{5}, v_{1}, v_{3}\right)+$ $\alpha \phi\left(v_{3}, v_{4}, v_{5}, v_{1}, v_{2}\right)$.

We shall denote the universal target of such maps $\phi$ by $V^{\lambda}$. That is, there is a map $i: V^{\times \lambda} \hookrightarrow V^{\lambda}$ satisfying (i)-(iii) such that, given any map $\phi: V^{\times \lambda} \rightarrow W$ satisfying (i)-(iii), there is a $\mathbb{D}$-linear map $\hat{\phi}: V^{\lambda} \rightarrow W$ with $\phi=\hat{\phi} \circ i$. This is called the Schur module of shape $\lambda$. In the next paragraph we shall show the existence of $V^{\lambda}$. Moreover, in Theorem 10.1 we shall see that, for a field $\mathbb{F}$, we have $V(\lambda)_{\mathbb{F}}=V_{\mathbb{F}}^{\lambda}$ if $\lambda$ has at most $n+1$ rows. In our situation this is satisfied always since $K \subseteq[n+1]$.

A concrete construction of $V^{\lambda} \quad$ All tensor and exterior products taken over boxes in $\lambda$ will be taken in order of the column word associated with $\lambda$; this word is obtained by concatenating the columns from left to right and by ordering the boxes in each column from bottom to top. ${ }^{1}$

We shall refer to this construction as the quotient construction of $V^{\lambda}$ since it realizes $V^{\lambda}$ as a quotient of $V^{\otimes \lambda}=V^{\otimes d}$ as follows. To enforce rules (i) and (ii), let $V^{\wedge \lambda}=\bigotimes_{k \in K}\left(\bigwedge^{k} V\right)^{\otimes l_{k}}$ and consider the canonical map $\pi_{\wedge}^{\times}: V^{\times \lambda} \rightarrow V^{\wedge \lambda}$ (which is the composition of a $\mathbb{D}$ - $d$-linear map $\pi_{\otimes}^{\times}: V^{\times \lambda} \rightarrow V^{\otimes \lambda}$ and a $\mathbb{D}$-linear map $\pi_{\wedge}^{\otimes}: V^{\otimes \lambda} \rightarrow V^{\wedge \lambda}$ ). To enforce rule (iii), let $Q^{\lambda}(V)$ be the subspace of $V^{\wedge \lambda}$ generated by all vectors $\pi_{\wedge}^{\times}(x)-\sum \pi_{\wedge}^{\times}(y)$, where $y$ runs through all vectors obtained from $x$ by an exchange between two given columns of $\lambda$ with a given set of boxes in the right chosen column. Then $Q^{\lambda}(V)$ is the kernel of the surjective $\mathbb{D}$-linear map

$$
\pi_{\lambda}^{\wedge}: V^{\wedge \lambda} \rightarrow V^{\lambda}
$$

We shall denote by $\pi_{\lambda}^{\otimes}: V^{\otimes \lambda} \rightarrow V^{\lambda}$ the canonical map $\pi_{\lambda}^{\wedge}$ o $\pi_{\wedge}^{\otimes}$.

Properties of $V^{\lambda} \quad$ A filling of $\lambda$ from $[m]=\{1,2, \ldots, m\}$ is a function $T: \lambda \rightarrow[m]$. Given any ordered set of vectors $\mathcal{B}=\left\{b_{1}, \ldots, b_{n+1}\right\}$, and a filling $T$ of $\lambda$ from $[n+1]$,

\footnotetext{
${ }^{1}$ In [23] the columns of $\lambda$ are numbered top to bottom.
} 
we get an element $b_{T}^{\times}$of $V^{\times \lambda}$ by setting, for each box $z$ of $\lambda$, its entry indexed by box $z$ equal to $b_{T(z)}$. Let

$$
b_{T}^{\otimes}=\pi_{\otimes}^{\times}\left(b_{T}^{\times}\right) \in V^{\otimes \lambda}, \quad b_{T}^{\wedge}=\pi_{\wedge}^{\otimes}\left(b_{T}^{\otimes}\right) \in V^{\wedge \lambda}, \quad b_{T}=\pi_{\lambda}^{\wedge}\left(b_{T}^{\wedge}\right) \in V^{\lambda} .
$$

We shall employ Lemma 10.1 in the case where $V$ is the natural module for $\mathfrak{s l}_{n+1}(\mathbb{D})$ and $\mathbb{D}$ is $\mathbb{Z}$ or a field $\mathbb{F}$.

Lemma 10.1 Suppose $V$ is a module for some Lie algebra $\mathfrak{g}_{\mathbb{D}}$ over $\mathbb{D}$. Then, the $\mathbb{D}$-modules $V^{\otimes \lambda}, V^{\wedge \lambda}$, and $V^{\lambda}$ are $\mathfrak{g}_{\mathbb{D}}$-modules as well.

Proof By equations (3) and (4) of Sect. 7 it follows that $V^{\otimes \lambda}$ and $V^{\wedge \lambda}$ are $\mathfrak{g}_{\mathbb{D}^{-}}$ modules. Now we show that $Q^{\lambda}(V)$ is a $\mathfrak{g}_{\mathbb{D}}$-submodule of $V^{\wedge \lambda}$.

Let $g \in \mathfrak{g}_{\mathbb{D}}$ and fix a subset $\mathcal{A}=\left\{a_{1}, \ldots, a_{n+1}, a_{n+2}=g a_{1}, \ldots, a_{2 n+2}=g a_{n+1}\right\}$ of $V$. Given a filling $T: \lambda \rightarrow[n+1]$, and a box $z \in \lambda$, let $T^{z}$ be obtained from $T$ by setting, for all boxes $y$ of $\lambda$ :

$$
T^{z}(y)= \begin{cases}T(y) & \text { if } y \neq z \\ T(z)+n+1 & \text { if } y=z\end{cases}
$$

Thus, $a_{T^{z}}^{\wedge}$ is obtained from $a_{T}^{\wedge}$ by replacing the contents of box $z$ by its $g$-image, so we have $g a_{T}^{\wedge}=\sum_{z \in \lambda} a_{T^{z}}^{\wedge}$. Now let $\xi$ be an exchange of $\lambda$, viewed as a permutation of the boxes in $\lambda$ and let $T^{\prime}$ be the filling obtained by composing $\xi$ and $T$, that is $T^{\prime}=T \circ \xi: \lambda \rightarrow[n+1]$. Given a filling $F: \lambda \rightarrow[n+1]$ we let $\xi \cdot a_{F}=a_{F \circ}$. Then,

$$
\begin{aligned}
& \xi g \cdot a_{T}^{\wedge}=\xi \sum_{z \in \lambda} a_{T^{z}}^{\wedge}=\sum_{z \in \lambda} a_{T^{z} \circ \xi}^{\wedge}, \\
& g \xi \cdot a_{T}^{\wedge}=g a_{T \circ \xi}^{\wedge}=\sum_{z^{\prime} \in \lambda} a_{(T \circ \xi)^{z^{\prime}}}^{\wedge} .
\end{aligned}
$$

It is straightforward to verify that $T^{z} \circ \xi=(T \circ \xi)^{\xi^{-1}(d)}$. Since $\xi$ is a permutation of $\lambda$ we can replace the last sum by $\sum_{\xi^{-1}(z)} a_{(T \circ \xi) \xi^{-1}(z)}^{\wedge}$ and conclude that $\xi g \cdot a_{T}^{\wedge}=$ $g \xi \cdot a_{T}^{\wedge}$. Thus,

$$
g\left(a_{T}-\sum_{\xi} \xi a_{T}\right)=g a_{T}-\sum_{\xi} g \xi a_{T}=g a_{T}-\sum_{\xi} \xi g a_{T},
$$

where the sum is taken over all exchanges between two given columns of $\lambda$ with a given set of boxes in the right chosen column. Hence, $Q^{\lambda}(V)$ is a $\mathfrak{g}_{\mathbb{D}}$-module. It follows that $V^{\lambda}=V^{\wedge \lambda} / Q^{\lambda}(V)$ is a $\mathfrak{g}_{\mathbb{D}}$-module as well.

From now on we shall assume that $V$ is free over $\mathbb{D}$.

We shall now discuss bases. Given a basis $\mathcal{B}$ for $V$, it is clear that $V^{\times \lambda}$ and $V^{\otimes \lambda}$ are free over $\mathbb{D}$. In fact, $\mathcal{B}^{\otimes \lambda}=\left\{b_{T}^{\otimes} \mid T\right\}$, where $T: \lambda \rightarrow[n+1]$ ranges over all possible fillings, is a $\mathbb{D}$-basis for $V^{\otimes \lambda}$. Moreover, it is clear that $V^{\wedge \lambda}$ is free over $\mathbb{D}$ with basis 
$\mathcal{B}^{\wedge \lambda}=\left\{b_{T}^{\wedge} \mid T\right\}$, where $T: \lambda \rightarrow[n+1]$ ranges over all fillings that are increasing down each column. Next, we identify certain bases of $V^{\lambda}$. A Young tableau of shape $\lambda$ is a filling $T$ of $\lambda$ that is weakly increasing along each row of $\lambda$ and strictly increasing down each column of $\lambda$. Let $\mathcal{B}^{\lambda}$ be the collection of all $b_{T}$, where $T$ ranges over all Young tableaux of shape $\lambda$ with entries from $[n+1]$.

Lemma 10.2 [23, §8.1, Theorem 1] Suppose $V$ is free over $\mathbb{D}$ with basis $\mathcal{B}=$ $\left\{b_{1}, \ldots, b_{n+1}\right\}$, then $V^{\lambda}$ is free over $\mathbb{D}$ with basis $\mathcal{B}^{\lambda}$.

Lemma 10.3 Let $V$ be a module for a Lie algebra $\mathfrak{g}_{\mathbb{D}}$ over $\mathbb{D}$. Suppose that $\mathcal{B}=$ $\left\{b_{1}, \ldots, b_{n+1}\right\}$ is a set of vectors such that $b_{i}$ has weight $\theta_{i}$ for each $i \in[n+1]$. Then, for any filling $T: \lambda \rightarrow[n+1]$, the vector $b_{T} \in V^{\lambda}$ has weight $\sum_{i=1}^{m} t_{i} \theta_{i}$, where $t_{i}$ is the number of occurrences of $b_{i}$ in $b_{T}$ and $m$ is the number of boxes in $\lambda$.

Proof This is a straightforward calculation.

The constructions of $V^{\lambda}$ as well as Lemmas 10.1, 10.2, and 10.3, show that if $V$ is a module for a (simple) Lie algebra $\mathfrak{g}_{\mathbb{C}}$ over $\mathbb{C}$ and $A$ is an admissible lattice, then we can construct $A^{\times \lambda}, A^{\otimes \lambda}, A^{\wedge \lambda}, Q^{\lambda}(A)$ and $A^{\lambda}$ replacing $V$ by $A$, taking $\mathbb{D}=\mathbb{Z}$ and viewing $A$ as a module for the Lie algebra $\mathfrak{g}_{\mathbb{D}}=\mathfrak{U}_{\mathbb{Z}}$ or $\mathfrak{g}_{\mathbb{D}}=\mathfrak{g}(\lambda)_{\mathbb{Z}}$. We summarize this and a little more in the following result.

Lemma 10.4 Let $V$ be a module for a simple Lie algebra $\mathfrak{g}_{\mathbb{C}}$ over $\mathbb{C}$ and suppose $V_{\mathbb{F}}=\mathbb{F} \otimes_{\mathbb{Z}}$ A for some admissible lattice $A$.

(a) The $\mathbb{Z}$-modules $A^{\times \lambda}, A^{\otimes \lambda}, A^{\wedge \lambda}$, and $A^{\lambda}$ are admissible lattices in $V^{\times \lambda}, V^{\otimes \lambda}$, $V^{\wedge \lambda}$, and $V^{\lambda}$, respectively.

(b) For any field $\mathbb{F}$ we have $\left(\mathbb{F} \otimes_{\mathbb{Z}} A\right)^{\lambda}=\mathbb{F} \otimes_{\mathbb{Z}} A^{\lambda}$. In particular, if we construct $V(\lambda)_{\mathbb{F}}=\mathbb{F} \otimes_{\mathbb{Z}} A^{\lambda}$, then $V(\lambda)_{\mathbb{F}} \cong V_{\mathbb{F}}^{\lambda}$.

(c) If $\mathcal{A}=\left\{a_{1}, \ldots, a_{n+1}\right\}$ is a $\mathbb{Z}$-basis of weight vectors for $A$, then $\mathcal{A}^{\lambda}$ is a $\mathbb{Z}$-basis of weight vectors for $A^{\lambda}$, where the weights are as described in Lemma 10.3.

Proof The admissible lattice $A$ is a free $\mathbb{Z}$-module as well as a module for the universal enveloping algebra $\mathfrak{U}_{\mathbb{Z}}$. The modules $A^{\times \lambda}, A^{\otimes \lambda}, A^{\wedge \lambda}$ and $A^{\lambda}$ can be constructed by replacing $V$ by $A$ and taking $\mathbb{D}=\mathbb{Z}$ in the construction of $V^{\lambda}$ above. Moreover,

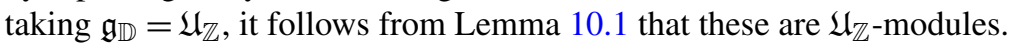

Let $\mathcal{A}$ be the basis in (c). As we saw in the discussion preceding Lemma 10.2, $\mathcal{A}$ canonically gives rise to bases $\mathcal{A}^{\otimes \lambda}$ and $\mathcal{A}^{\wedge \lambda}$ for $A^{\otimes \lambda}$, and $A^{\wedge \lambda}$, respectively. By Lemma 10.2 since $\mathcal{A}$ is a basis for $V, \mathcal{A}^{\lambda}$ is a basis for $V^{\lambda}$ so in particular, $\mathcal{A}^{\lambda}$ is independent over $\mathbb{Z}$ and of appropriate cardinality. The fact that $\mathcal{A}^{\lambda}$ spans $A^{\lambda}$ over $\mathbb{Z}$ follows from the fact that $\mathcal{A}^{\otimes \lambda}$ spans $A^{\otimes \lambda}$ and $A^{\lambda}$ is a quotient of $A^{\otimes \lambda}$. Thus (a) follows. Part (b) is a special case of the remark preceding Lemma 1 in [23, §8.1]. Part (c) follows from Lemma 10.3.

Remark 10.1 If in Lemma 10.4 we have $\mathfrak{g}_{\mathbb{C}}=\mathfrak{s l}_{n+1}(\mathbb{C})$, then the natural module $V$ has a basis $\mathcal{A}=\left\{a_{1}, \ldots, a_{n+1}\right\}$ whose weights satisfy $\theta_{1} \succ \theta_{2} \succ \cdots \succ \theta_{n+1}$ in the 
natural ordering on weights. Namely, $\theta_{i}-\theta_{i+1}=\alpha_{i}$, the $i$ th fundamental root, for $i=1, \ldots, n$. It then follows that the highest weight vector of $V^{\lambda}$ is $a_{T}$, where $T$ is the tableau whose $i$ th row is filled with $i$ 's only. In view of the relation between weights and Young diagrams, and using that $\theta_{1}+\cdots+\theta_{k}=\lambda_{k}$ for any $1 \leq k \leq n$, we find that $a_{T}$ has weight $\lambda$.

Theorem 10.1 (Theorem 8.2 of [23]) If $\lambda$ has at most $n+1$ rows and $\mathbb{F}=\mathbb{C}$, then $V^{\lambda}$ is an irreducible representation of highest weight $\lambda$ for $\mathrm{GL}_{n+1}(\mathbb{F})$. These are all irreducible polynomial representations of $\mathrm{GL}_{n+1}(\mathbb{F})$.

Corollary 10.1 For any field $\mathbb{F}$, the $\mathfrak{s l}_{n+1}(\mathbb{F})$-modules $V(\lambda)_{\mathbb{F}}$ and $V_{\mathbb{F}}^{\lambda}$ are isomorphic.

Proof Since $\lambda$ was constructed from the subset $K \subseteq[n+1]$, all $V_{\mathbb{C}}^{\lambda}$ constructed in this section are irreducible $\mathrm{GL}_{n+1}(\mathbb{C})$-modules. Since $\mathrm{GL}_{n+1}(\mathbb{C})$ is a central extension of $\mathrm{SL}_{n+1}(\mathbb{C})$ it follows that $V_{\mathbb{C}}^{\lambda}$ is also irreducible as an $\mathrm{SL}_{n+1}(\mathbb{C})$-module. Hence $V_{\mathbb{C}}^{\lambda}$ is naturally an irreducible $\mathfrak{s l}_{n+1}(\mathbb{C})$-module.

The well-known classification of finite dimensional irreducible modules for simple complex Lie algebras (see e.g. [15, Chap. 10]) in particular ensures that, for each $\lambda$ (which in our case is integral and dominant), there is a unique irreducible $\mathfrak{s l}_{n+1}(\mathbb{C})$ module of highest weight $\lambda$. Since $V_{\mathbb{C}}^{\lambda}$ is an irreducible $\mathfrak{s l}_{n+1}(\mathbb{C})$-module of highest weight $\lambda, L(\lambda) \mathbb{C}=V(\lambda) \mathbb{C}=V_{\mathbb{C}}^{\lambda}$.

By Lemma 10.4 if $A$ is an admissible lattice in $V$, then $A^{\lambda}$ is an admissible lattice in $V^{\lambda}$. The result follows, since by part (b) of that lemma, $V(\lambda)_{\mathbb{F}}=\mathbb{F} \otimes_{\mathbb{Z}} A^{\lambda}=$ $\left(\mathbb{F} \otimes_{\mathbb{Z}} A\right)^{\lambda}=V_{\mathbb{F}}^{\lambda}$, for any field $\mathbb{F}$.

Note that by Lemma 10.2 (and 10.4) the dimension of $V^{\lambda}$ is independent of the field $\mathbb{F}$. This is a special case of Proposition 4.1 parts (b) and (c).

As already noted in Remark $4.7, V^{\lambda}$ is in general not cyclic.

The Weyl embedding Now let $\lambda=\lambda_{K}$ be as in Sect. 4.2, and $\mathbb{F}$ some field. Consider the following point of $\Gamma: p=\left(A_{k}\right)_{k \in K}$, where $A_{k}=\left\langle a_{1}, \ldots, a_{k}\right\rangle_{V}$. Then by Theorem 4.2 the Weyl embedding satisfies

$$
\begin{aligned}
e_{W}: \mathcal{P} & \rightarrow \mathbb{P}\left(\left(V^{\lambda}\right)^{0}\right), \\
p & \mapsto a_{T}
\end{aligned}
$$

where $T$ is the Young tableau whose $i$ th row is filled with $i$ 's only. That is $a_{T}=$ $\pi_{\lambda}^{\wedge}\left(\bigotimes_{k \in K} \bigwedge_{i=1}^{k} a_{i}\right)$. For any subset $K \subseteq I$, let $e_{K}$ be the Weyl embedding of the $K$-shadow space of $\Delta$. Then we have

$$
e_{K}(p)=\pi_{\lambda}^{\wedge}\left(\bigotimes_{k \in K} e_{k}\left(A_{k}\right)\right)
$$

Now note that $\pi_{\lambda}^{\wedge}$ is a $G$-module homomorphism and that $G$ is transitive on the points of $\Gamma$. Therefore we have equality (7) for any point $p=\left(A_{k}\right)_{k \in K}$ of $\Gamma$. 
The form $\beta$ and a second construction of $V^{\lambda} \quad$ We first show that the contravariant form $\beta$ on $V^{\wedge \lambda}$ and $V^{\lambda}$ can be obtained from the contravariant form on $V$. Recall that $\beta$ is constructed initially on the module $V(\lambda) \mathbb{C}$, then restricted to an admissible lattice, and then tensored with $\mathbb{F}$ to get its equivalent on $V(\lambda)_{\mathbb{F}}$. Thus in order to describe $\beta$, we can and shall work over $\mathbb{C}$. Our aim is to find a result similar to part (a) of Lemmas 7.1 and 7.2. To this end it is convenient to use the Schur functor (see e.g. [23, §8.3] and [24]).

We first use the Schur functor (and its dual) to describe $V^{\lambda}$ and $\left(V^{*}\right)^{\lambda}$. We shall consider $\left(V^{*}\right)^{\lambda}$ to be constructed from $V^{*}$ in the same way as $V^{\lambda}$ is constructed from $V$ and denote the maps corresponding to $\pi_{\lambda}^{\times}, \pi_{\lambda}^{\wedge}, \pi_{\lambda}^{\otimes}$ by putting a bar over them, so that we get $\bar{\pi}_{\lambda}^{\times}, \bar{\pi}_{\lambda}^{\wedge}$, and $\bar{\pi}_{\lambda}^{\otimes}$. Fix $\lambda$ and let it have $d$ boxes. Let $\mathbf{C}=\mathbb{C}\left[S_{d}\right]$, where $S_{d}$ is the symmetric group on the set $[d]$. In the sequel, for any right $\mathbf{C}$-module $A$ and left $\mathbf{C}$-module $B$, the tensor product $A \otimes_{\mathbf{C}} B$ is the quotient of the usual tensor product $A \otimes_{\mathbb{C}} B$ over $\mathbb{C}$ by the subspace generated by all expressions

$$
(a \cdot \sigma) \otimes b-a \otimes(\sigma \cdot b) \quad \text { where } a \in A, \sigma \in S_{d}, b \in B .
$$

We view $V^{\otimes d}$ (resp. $\left(V^{*}\right)^{\otimes d}$ ) as a left (resp. right) $\mathbb{C}$-vector space and a right (resp. left) C-module, where $S_{d}$ naturally (resp. reverse) permutes the components of $V^{\otimes d}$ (resp. $\left.\left(V^{*}\right)^{\otimes d}\right)$.

Then, we have natural isomorphisms $V^{\otimes d} \cong V^{\otimes d} \otimes_{\mathbf{C}} \mathbf{C}$ and $\left(V^{*}\right)^{\otimes d} \cong \mathbf{C} \otimes_{\mathbf{C}}$ $\left(V^{*}\right)^{\otimes d}$. Note that the natural pairing $p: V \times V^{*} \rightarrow \mathbb{C}$ given by $\langle v, f\rangle=f(v)$ gives rise to the pairing $p^{\otimes}: V^{\otimes d} \times\left(V^{*}\right)^{\otimes d} \rightarrow \mathbb{C}$ by setting $\left\langle v_{1} \otimes \cdots \otimes v_{d}, f_{1} \otimes\right.$ $\left.\cdots \otimes f_{d}\right\rangle=\prod_{i=1}^{d} f_{i}\left(v_{i}\right)$, thus identifying $\left(V^{*}\right)^{\otimes d}$ and $\left(V^{\otimes d}\right)^{*}$. Note here that we use the conventional notation $f(v)$ even though $V^{*}$ is a right vector space. It follows from the above definitions that we now have

$$
\langle v \otimes c \sigma, 1 \otimes f\rangle=\langle v \otimes 1, c \sigma \otimes f\rangle
$$

for any $f \in\left(V^{*}\right)^{\otimes d}, v \in V^{\otimes d}, c \in \mathbb{C}$, and $\sigma \in S_{d}$. Here the pairing $\langle\cdot, \cdot \cdot\rangle$ is induced by $p^{\otimes}$ via the natural isomorphisms $V^{\otimes d} \cong V^{\otimes d} \otimes_{\mathbf{C}} \mathbf{C}$ and $\left(V^{*}\right)^{\otimes d} \cong \mathbf{C} \otimes_{\mathbf{C}}\left(V^{*}\right)^{\otimes d}$.

A numbering $U$ of $\lambda$ is a filling from $[d]$ without repeated entries. Let $U$ be the numbering of $\lambda$ that agrees with the natural ordering of boxes of $\lambda$ taken in the quotient construction of $V^{\lambda}$, that is, so that the column word of $U$ is $1,2, \ldots, d$. Let $R(U)$ and $C(U)$ be the row group and column group of $U$; that is, $R(U)$ (resp. $C(U)$ ) is the subgroup of $S_{d}$ that simultaneously preserves the subsets of numbers in $U$ associated with the rows (resp. columns) of $\lambda$. Let

$$
\rho_{U}=\sum_{\rho \in R(U)} \rho, \quad \gamma_{U}=\sum_{\gamma \in C(U)} \operatorname{sign}(\gamma) \gamma, \quad \sigma_{U}=\gamma_{U} \rho_{U}, \quad \bar{\sigma}_{U}=\rho_{U} \gamma_{U} .
$$

The Specht module $S^{\lambda}$ with diagram $\lambda$ can be identified with the right $\mathbf{C}$-module $\sigma_{U} \mathbf{C}$ as well as the left $\mathbf{C}$-module $\mathbf{C} \sigma_{U}$ (see e.g. [24, Chap. 4]). Using the Schur functor, i.e. tensoring with the Specht module, we have isomorphisms

$$
\begin{aligned}
& V^{\lambda} \cong V^{\otimes d} \otimes_{\mathbf{C}} \mathbf{C} \sigma_{U}, \\
& \pi_{\lambda}^{\otimes}\left(v_{1} \otimes \cdots \otimes v_{d}\right) \mapsto v_{1} \otimes \cdots \otimes v_{d} \otimes_{\mathbf{C}} \sigma_{U}, \quad \text { and }
\end{aligned}
$$




$$
\begin{gathered}
\left(V^{*}\right)^{\lambda} \cong \sigma_{U} \mathbf{C} \otimes_{\mathbf{C}}\left(V^{*}\right)^{\otimes d}, \\
\bar{\pi}_{\lambda}^{\otimes}\left(f_{1} \otimes \cdots \otimes f_{d}\right) \mapsto \frac{1}{k_{\lambda}} \sigma_{U} \otimes_{\mathbf{C}} f_{1} \otimes \cdots \otimes f_{d},
\end{gathered}
$$

where $k_{\lambda}$ is the integer such that $\sigma_{U}^{2}=k_{\lambda} \sigma_{U}$. The quotient construction of $V^{\lambda}$ corresponds exactly to applying the Schur functor as above since by choice of $U$, we have

$$
\begin{aligned}
& V^{\otimes \lambda}=V^{\otimes d} \otimes_{\mathbf{C}} \mathbf{C} \stackrel{\pi_{\wedge}^{\otimes}}{\rightarrow} V^{\wedge \lambda}=V^{\otimes d} \otimes_{\mathbf{C}} \mathbf{C} \gamma_{U} \stackrel{\pi_{\lambda}^{\wedge}}{\rightarrow} V^{\lambda}=V^{\otimes d} \otimes_{\mathbf{C}} \mathbf{C} \sigma_{U}, \\
& v_{1} \otimes \cdots \otimes v_{d} \otimes_{\mathbf{C}} 1 \mapsto v_{1} \otimes \cdots \otimes v_{d} \otimes_{\mathbf{C}} \gamma_{U} \mapsto v_{1} \otimes \cdots \otimes v_{d} \otimes_{\mathbf{C}} \gamma_{U} \rho_{U}
\end{aligned}
$$

and $\pi_{\lambda}^{\otimes}=\pi_{\lambda}^{\wedge} \circ \pi_{\wedge}^{\otimes}$ (cf. [23, §8.3]). In the construction of $\left(V^{*}\right)^{\lambda}$ one could use $\bar{\sigma}_{U}$ instead of $\frac{1}{k_{\lambda}} \sigma_{U}$. Our choice is more natural, as we will see in the proof of the following general lemma.

Lemma 10.5 We have an isomorphism $\left(V^{\lambda}\right)^{*} \cong\left(V^{*}\right)^{\lambda}$ induced by the pairing: $p^{\lambda}: V^{\lambda} \times\left(V^{*}\right)^{\lambda} \rightarrow \mathbb{C}$ given by

$$
\left\langle\pi_{\lambda}^{\otimes}\left(v_{1} \otimes \cdots \otimes v_{d}\right), \bar{\pi}_{\lambda}^{\otimes}\left(f_{1} \otimes \cdots \otimes f_{d}\right)\right\rangle=\sum_{\rho \in R(U)} \sum_{\gamma \in C(U)} \operatorname{sign}(\gamma) \prod_{i=1}^{d} f_{i}\left(v_{(\gamma \rho)(i)}\right),
$$

where $U$ is as above.

Proof Consider the isomorphisms

$$
\begin{gathered}
V^{\lambda} \cong V^{\otimes d} \otimes_{\mathbf{C}} \mathbf{C} \sigma_{U}, \\
\left(V^{*}\right)^{\lambda} \cong \sigma_{U} \mathbf{C} \otimes_{\mathbf{C}}\left(V^{*}\right)^{\otimes d} .
\end{gathered}
$$

It can be shown that $\sigma_{U}^{2}=k_{\lambda} \sigma_{U}$, for some non-zero $k_{\lambda} \in \mathbb{Z}$, so that, for $v \in V^{\otimes d}$ and $f \in\left(V^{*}\right)^{\otimes d}$, we have

$$
\left\langle v \otimes_{\mathbf{C}} \sigma_{U}, 1 \otimes_{\mathbf{C}} f\right\rangle=\left\langle v \otimes_{\mathbf{C}} \frac{1}{k_{\lambda}} \sigma_{U}^{2}, 1 \otimes_{\mathbf{C}} f\right\rangle .
$$

It follows from (8) that the pairing $p^{\otimes}$ has the property that,

$$
\left\langle v \otimes_{\mathbf{C}} \frac{1}{k_{\lambda}} \sigma_{U}^{2}, 1 \otimes_{\mathbf{C}} f\right\rangle=\left\langle v \otimes_{\mathbf{C}} \sigma_{U}, \frac{1}{k_{\lambda}} \sigma_{U} \otimes_{\mathbf{C}} f\right\rangle .
$$

We have maps:

$$
V^{\otimes d} \otimes_{\mathbf{C}} \mathbf{C} \sigma_{U} \stackrel{i}{\hookrightarrow} V^{\otimes d} \otimes_{\mathbf{C}} \mathbf{C} \stackrel{\pi}{\rightarrow} V^{\otimes d} \otimes_{\mathbf{C}} \mathbf{C} \sigma_{U}
$$

where $i$ is the identity and $\pi$ is right multiplication by $\sigma_{U}$. The surjective map $\pi$ induces an injection $\pi^{*}:\left(V^{\otimes d} \otimes_{\mathbf{C}} \mathbf{C} \sigma_{U}\right)^{*} \hookrightarrow\left(V^{\otimes d} \otimes_{\mathbf{C}} \mathbf{C}\right)^{*}$. Since $\sigma_{U}^{2}=k_{\lambda} \sigma_{U}$, we 
have $\pi \circ i=k_{\lambda}$ id so that for every $g \in\left(V^{\otimes d} \otimes_{\mathbf{C}} \mathbf{C} \sigma_{U}\right)^{*}$ we have $g=\frac{1}{k_{\lambda}} i^{*} \circ \pi^{*}(g)$. Thus $i^{*}:\left(V^{\otimes d} \otimes_{\mathbf{C}} \mathbf{C}\right)^{*} \rightarrow\left(V^{\otimes d} \otimes_{\mathbf{C}} \mathbf{C} \sigma_{U}\right)^{*}$, given by $f \mapsto f \circ i$, induces the inverse to $\frac{1}{k_{\lambda}} \pi^{*}$ on the image of $\pi^{*}$. To see what this means, we identify $V^{\otimes d} \otimes_{\mathbf{C}} \mathbf{C} \sigma_{U}$ with its image under $i$. Then $g=\frac{1}{k_{\lambda}} i^{*} \circ \pi^{*}(g)$ says that $g \in\left(V^{\otimes d} \otimes_{\mathbf{C}} \mathbf{C} \sigma_{U}\right)^{*}$ is simply the restriction of some element of $\left(V^{\otimes d} \otimes_{\mathbf{C}} \mathbf{C}\right) *$ (namely $\frac{1}{k_{\lambda}} \pi^{*}(g)$ ). Now use the pairing $p^{\otimes}$ to view $\mathbf{C} \otimes_{\mathbf{C}}\left(V^{*}\right)^{\otimes d} \cong\left(V^{\otimes d} \otimes_{\mathbf{C}} \mathbf{C}\right)^{*}$. Namely define a map $b^{\otimes}$ by setting $c \otimes_{\mathbf{C}} f \mapsto\left\langle\cdot, c \otimes_{\mathbf{C}} f\right\rangle$, for all $c \in \mathbf{C}$ and $f \in\left(V^{*}\right)^{\otimes d}$. Then, from (9) and (10) we can see that if this element $g$ is represented by some $f \in \mathbf{C} \otimes_{\mathbf{C}}\left(V^{*}\right)^{\otimes d}$, then it is also represented by $\frac{1}{k_{\lambda}} \sigma_{U} \otimes f \in \sigma_{U} \mathbf{C} \otimes_{\mathbf{C}}\left(V^{*}\right)^{\otimes d}$. Hence, $i^{*} \circ b^{\otimes}$ is an isomorphism between $\sigma_{U} \mathbf{C} \otimes_{\mathbf{C}}\left(V^{*}\right)^{\otimes d}$ and $\left(V^{\otimes d} \otimes_{\mathbf{C}} \mathbf{C} \sigma_{U}\right)^{*}$. The definition of $\pi_{\lambda}^{\otimes}, \bar{\pi}_{\lambda}^{\otimes}$ and $b^{\otimes}$ imply that, for $v \in V^{\otimes d}$ and $f \in\left(V^{*}\right)^{\otimes d}$ we have $\left\langle\pi_{\lambda}^{\otimes}(v), \bar{\pi}_{\lambda}^{\otimes}(f)\right\rangle=\left\langle v \otimes \sigma_{U}, 1 \otimes f\right\rangle$. The conclusion of the lemma now follows from the meaning of $v_{1} \otimes \cdots \otimes v_{d} \otimes \sigma_{U}$ and the standard pairing $p^{\otimes}$.

If $V$ is a module for a simple Lie algebra $\mathfrak{g}_{\mathbb{C}}$ over $\mathbb{C}$, and $\beta$ is a $\tau$-contravariant form on $V$, we shall denote the forms on $V^{\otimes \lambda}$ and $V^{\wedge \lambda}$ defined using Lemmas 7.1 and 7.2 by $\beta^{\otimes \lambda}$ and $\beta^{\wedge \lambda}$. It follows that $\beta^{\wedge \lambda}$ is $\tau$-contravariant. We now extend this to a form on $V^{\lambda}$.

Lemma 10.6 Let $V$ be a finite dimensional $\mathbb{F}$-vector space, let $\sigma$ be an automorphism of $\mathbb{F}$ of order at most 2 and let $\zeta$ be a $\sigma$-sesquilinear form on $V$. Let $k \in \mathbb{N}_{\geq 1}$. Moreover, let $\mathbf{C}=\mathbb{F}\left[S_{d}\right]$ and let $U$ and $\sigma_{U}$ be as above. Then

(a) there is a unique $\sigma$-sesquilinear form $\zeta^{\lambda}$ on $V^{\otimes d} \otimes_{\mathbf{C}} \mathbf{C} \sigma_{U}$ given by

$$
\begin{aligned}
& \zeta^{\lambda}\left(v_{1} \otimes \cdots \otimes v_{d} \otimes_{\mathbf{C}} \sigma_{U}, u_{1} \otimes \cdots \otimes u_{d} \otimes_{\mathbf{C}} \sigma_{U}\right) \\
& \quad=\sum_{\gamma \in C(U)} \sum_{\rho \in R(U)} \operatorname{sign}(\gamma) \sum_{\tilde{\gamma} \in C(U)} \operatorname{sign}(\tilde{\gamma}) \prod_{i=1}^{d} \zeta\left(v_{(\gamma \rho \tilde{\gamma})(i)}, u_{i}\right) ;
\end{aligned}
$$

(b) if $\zeta$ is symmetric bilinear, so is $\zeta^{\lambda}$;

(c) if $V$ is a finite dimensional module for a simple Lie algebra $\mathfrak{g}_{\mathbb{F}}$ and $\zeta$ is $\tau$ contravariant, then so is $\zeta^{\lambda}$.

Proof (a) The form $\zeta$ can be obtained by composing the standard pairing $V \times V^{*} \rightarrow \mathbb{F}$ given by $\langle v, f\rangle=f(v)$ with a $\sigma$-semilinear homomorphism $\phi: V \rightarrow V^{*}$ so that $\zeta(v, u)=\langle v, \phi(u)\rangle$. Now the following composition that we shall call $\phi^{\lambda}$ is again a $\sigma$-semilinear homomorphism; note that if $\phi$ is an isomorphism, then so is $\phi^{\lambda}$.

$$
\begin{aligned}
V^{\otimes d} \otimes_{\mathbf{C}} \mathbf{C} \sigma_{U} & \rightarrow V^{\otimes d} \otimes_{\mathbf{C}} \mathbf{C} \bar{\sigma}_{U} \rightarrow \sigma_{U} \mathbf{C} \otimes_{\mathbf{C}}\left(V^{*}\right)^{\otimes d}, \\
u_{1} \otimes \cdots \otimes u_{d} \otimes_{\mathbf{C}} \sigma_{U} & \rightarrow u_{1} \otimes \cdots \otimes u_{d} \otimes_{\mathbf{C}} \frac{1}{k_{\lambda}} \gamma_{U} \bar{\sigma}_{U} \\
& \rightarrow \frac{1}{k_{\lambda}} \sigma_{U} \gamma_{U} \otimes_{\mathbf{C}} \phi\left(u_{1}\right) \otimes \cdots \otimes_{\mathbf{C}} \phi\left(u_{d}\right) .
\end{aligned}
$$


Namely, the first map is the isomorphism given by right multiplication by $\frac{1}{k_{\lambda}} \gamma_{U}$, whose inverse is given by multiplication on the right by $\rho_{U}$. The second map combines $\phi^{\otimes d}$ with the isomorphism $\sum_{x \in S_{d}} a_{x} x \mapsto \sum_{x \in S_{d}} a_{x} x^{-1}$ between the left and right regular representation of $\mathbf{C}$. Note that $C(U)$ and $R(U)$ are closed under taking inverses so that $\gamma_{U} \rho_{U} \gamma_{U}$ is invariant under taking inverses. Note here that

$$
\phi^{\lambda}\left(u_{1} \otimes \cdots \otimes u_{d} \otimes_{\mathbf{C}} \sigma_{U}\right)=\sum_{\tilde{\gamma} \in C(U)} \operatorname{sign}(\tilde{\gamma}) \bar{\pi}^{\lambda}\left(\phi\left(u_{\tilde{\gamma}^{-1}(1)}\right) \otimes \cdots \otimes \phi\left(u_{\tilde{\gamma}^{-1}(d)}\right)\right)
$$

We then set $\zeta^{\lambda}=p^{\lambda}\left(\cdot, \phi^{\lambda}(\cdot)\right)$. It is immediate from this construction that $\zeta^{\lambda}$ is $\sigma$ sesquilinear. In order to obtain the formula we use that $\prod_{i=1}^{d} \zeta\left(v_{(\gamma \rho)(i)}, \phi\left(u_{\tilde{\gamma}^{-1}(i)}\right)\right)=$ $\prod_{j=1}^{d} \zeta\left(v_{(\gamma \rho \tilde{\gamma})(j)}, \phi\left(u_{j}\right)\right)$.

(b) Note that if $\zeta$ is symmetric, then $\zeta\left(v_{(\gamma \rho \tilde{\gamma})(i)}, u_{i}\right)=\zeta\left(u_{\left(\tilde{\gamma}^{-1} \rho^{-1} \gamma^{-1}\right)(j)}, v_{j}\right)$ for $j=\gamma \rho \tilde{\gamma}(i)$. Since the sums are taken over all $\gamma, \tilde{\gamma} \in C(U)$ and $\rho \in R(U)$ and these are closed under taking inverses, we find that $\zeta^{\lambda}$ is symmetric as well. (c) This follows from part (e) of Lemma 7.1.

Lemma 10.7 Suppose $\mathcal{A}=\left\{a_{1}, \ldots, a_{n}\right\}$ is an orthonormal basis for $V$ with respect to $\zeta$ and $T$ is the Young tableau of shape $\lambda$ whose ith row is filled with $i$ 's only. Then, $\zeta^{\lambda}\left(a_{T}, a_{T}\right)=k_{\lambda}$. Moreover, $\zeta^{\lambda}\left(a_{T}, a_{T^{\prime}}\right)=0$ for any Young tableau $T^{\prime} \neq T$ of shape $\lambda$.

Proof In view of Lemma 10.6 we have

$$
\begin{aligned}
\zeta^{\lambda}\left(a_{T}, a_{T}\right) & =\sum_{\gamma \in C(U)} \sum_{\rho \in R(U)} \sum_{\tilde{\gamma} \in C(U)} \operatorname{sign}(\gamma) \operatorname{sign}(\tilde{\gamma}) \prod_{i=1}^{d} \zeta\left(a_{(\gamma \rho \tilde{\gamma})(i)}, a_{i}\right) \\
& =\sum_{\gamma \in C(U)} \sum_{\rho \in R(U)} \sum_{\tilde{\gamma} \in C(U)} \operatorname{sign}(\gamma) \operatorname{sign}(\tilde{\gamma}),
\end{aligned}
$$

where the sum is taken over those $\gamma, \rho, \tilde{\gamma}$ such that $\gamma \rho \tilde{\gamma} \tilde{\rho}=1$ for some $\tilde{\rho} \in R(U)$. This is because $\zeta\left(a_{(\gamma \rho \tilde{\gamma})(i)}, a_{i}\right)=1$ if and only if $a_{(\gamma \rho \tilde{\gamma})(i)}$ and $a_{i}$ are in the same row and 0 otherwise. Now consider the equation $\gamma_{U} \rho_{U} \gamma_{U} \rho_{U}=k_{\lambda} \gamma_{U} \rho_{U}$. The coefficient of $1 \in \mathbf{C}$ on the right hand side is exactly the sum above and it clearly equals $k_{\lambda}$.

Now if $T^{\prime} \neq T$ is a Young tableau with the same Young diagram as $\lambda$ then for any permutation $\sigma \in S_{d}$, the fillings $\sigma T$ and $T^{\prime}$ differ in some box. Since $\mathcal{A}$ is orthonormal, this means that $\prod_{i=1}^{d} \zeta\left(a_{\sigma(i)}, a_{i}\right)=0$. It follows that $\zeta^{\lambda}\left(a_{T}, a_{T^{\prime}}\right)=0$.

Corollary 10.2 Let $\beta$ be the symmetric bilinear $\tau$-contravariant form on $V$ as defined in Sect. 5.2. Then $\frac{1}{k_{\lambda}} \beta^{\lambda}$ is the symmetric bilinear $\tau$-contravariant form on $V^{\lambda}$.

Proof This follows from Remark 10.1, Lemmas 10.6 and 10.7. 


\subsection{An illustration}

We illustrate what happens in this section with a well-known example [47]. Let $\Delta$ be the building of type $A_{2}$ and let $\Gamma$ be the $\{1,2\}$-shadow space of $\Delta$ over a field $\mathbb{F}$.

We have $\mathfrak{g}_{\mathbb{C}}=\mathfrak{s l}_{3}(\mathbb{C})$, which acts on its natural module $V$ by matrix-vector multiplication from the left, where vectors are coordinate vectors with respect to a basis $\mathcal{A}=\left\{a_{1}, a_{2}, a_{3}\right\}$. Writing $X_{i, j}$ for the elementary matrix whose non-zero entry is a 1 in the $(i, j)$ position, and setting $H_{i, j}=X_{i, i}-X_{j, j}$ and $Y_{i, j}=X_{j, i}$ for $i<j$, we have a Chevalley basis

$$
\mathrm{C}=\left\{X_{i, j} \mid i<j\right\} \cup\left\{H_{1,2}, H_{2,3}\right\} \cup\left\{Y_{i, j} \mid i<j\right\} .
$$

The multiplication is given by $\left[X_{i, j}, X_{k, l}\right]=\delta_{j, k} X_{i, l}-\delta_{l, i} X_{k, j}$ for all $i, j, k, l$. Now, in terms of Young diagrams, we have $\lambda=(2,1)$ and $V_{\mathbb{C}}^{\lambda}$ is the adjoint representation, i.e. it is $\mathfrak{s l}_{3}(\mathbb{C})$ itself under the action ad $x(y)=x y-y x$. The highest weight vector is $v^{+}=X_{1,3}$ and the minimal admissible lattice $A_{\min }$ is the $\mathbb{Z}$-span of the Chevalley basis C. Writing $a_{i, j, k}=a_{i} \otimes a_{j} \otimes a_{k} \otimes \sigma_{U}$, and comparing the $\mathfrak{s l}_{3}(\mathbb{C})$ action on itself with that on $V_{\mathbb{C}}^{\lambda}$, we find that the Chevalley basis elements are identified with elements of $V^{\otimes 3} \otimes_{\mathbf{C}} \mathbf{C} \sigma_{U}$ as follows:

$$
\begin{array}{lll}
X_{1,3}=a_{2,1,1}, & H_{1,2}=a_{2,1,3}-2 a_{3,1,2}, & Y_{1,3}=a_{3,2,3}, \\
X_{1,2}=-a_{3,1,1}, & H_{2,3}=a_{2,1,3}+a_{3,1,2}, & Y_{1,2}=a_{3,2,2}, \\
X_{2,3}=a_{2,1,2}, & & Y_{2,3}=-a_{3,1,3} .
\end{array}
$$

We also have $H_{1,3}=2 a_{2,1,3}-a_{3,1,2}$. If $\mathcal{A}$ is orthonormal with respect to $\beta$, then by direct calculation from Lemma 10.6 one verifies that $\beta^{\lambda}$ is given with respect to the Chevalley basis $\mathrm{C}$ by the following matrix

$$
B=\left(\begin{array}{cccccccc}
1 & & & & & & & \\
& 1 & & & & & & \\
& & 1 & & & & & \\
& & & 2 & -1 & & & \\
& & & -1 & 2 & & & \\
& & & & & 1 & & \\
& & & & & & 1 & \\
& & & & & & & 1
\end{array}\right) .
$$

Note that $B$ has full rank in all characteristics except 3, where it has rank 7 . In that case $H_{1,2}-H_{2,3}$ spans the radical of $\beta^{\lambda}$. Note also that this form can be given as $\beta^{\lambda}(x, y)=\operatorname{trace}\left(x y^{\tau}\right)$, for all $x, y \in \mathfrak{s l}_{3}(\mathbb{C})$ where $y^{\tau}$ denotes the transpose of $y$.

Now consider the geometric picture. Let $\mathbb{F}$ be an arbitrary field. The shadow space $\Gamma$ is the geometry of point-line flags $(p, l)$ of $\operatorname{PG}\left(V_{\mathbb{F}}\right)$. Identifying $V_{\mathbb{F}}^{\lambda}$ with the adjoint module $\mathfrak{s l}_{3}(\mathbb{F})$, the Weyl embedding is given by

$$
\begin{gathered}
\mathcal{P} \hookrightarrow \operatorname{PG}\left(V_{\mathbb{F}}^{\lambda}\right), \\
(p, l) \mapsto\left\langle w_{p, l}=v_{p} n_{l}^{\tau}\right\rangle,
\end{gathered}
$$


where $v_{p}$ is some (column) vector spanning $p$ and $n_{l}$ is some (column) vector that is orthogonal to $l$ with respect to the inner product $\beta$ and $\tau$ denotes transposition. Note that trace $\left(w_{p, l}\right)=0$ since $p$ lies on $l$. Considering the apartment $\Sigma$ of $\Delta$ given by the basis $\mathcal{A}$, we find that $\left(\left\langle a_{i}\right\rangle,\left\langle a_{i}, a_{k}\right\rangle\right) \mapsto\left\langle a_{k} \otimes a_{i} \otimes a_{i} \otimes \sigma_{U}\right\rangle=\left\langle a_{k, i, i}\right\rangle=\left\langle X_{i, j}\right\rangle$, where $\{i, j, k\}=\{1,2,3\}$. In particular $\left(\left\langle a_{1}\right\rangle,\left\langle a_{1}, a_{2}\right\rangle\right)$ is sent to the space $\left\langle a_{2,1,1}\right\rangle=\left\langle v^{+}\right\rangle$ of highest weight $\lambda=\lambda_{1}+\lambda_{2}$.

We now look at singular hyperplanes. Two point-line flags $\left(p_{1}, l_{1}\right)$ and $\left(p_{2}, l_{2}\right)$ are opposite if and only if $p_{1} \notin l_{2}$ and $p_{2} \notin l_{1}$. Considering the matrices $w_{p_{1}, l_{1}}=$ $v_{p_{1}} n_{l_{1}}^{\tau}$ and $w_{p_{2}, l_{2}}=v_{p_{2}} n_{l_{2}}^{\tau}$ we find that $\left(p_{1}, l_{1}\right)$ and $\left(p_{2}, l_{2}\right)$ are opposite if and only if trace $\left(w_{p_{1}, l_{1}} w_{p_{2}, l_{2}}\right) \neq 0$. The singular hyperplane $H(p, l)$ of $\Gamma$ of points not opposite to $(p, l) \in \Gamma^{*}=\Gamma$ is therefore induced by the hyperplane $\operatorname{ker}\left(\operatorname{trace}\left(w_{p, l} * \cdot\right)\right)$ of $\operatorname{PG}\left(V_{\mathbb{F}}^{\lambda}\right)$. It follows therefore that the polar radical of $\operatorname{PG}\left(V_{\mathbb{F}}^{\lambda}\right)$ is the radical of the symmetric bilinear form $\zeta(x, y)=\operatorname{trace}(x y)$. Since the transpose map $\tau$ simply sends the image of $\Gamma^{*}=\Gamma$ to that of $\Gamma$, we see once again that $\operatorname{Rad}(\zeta)=\operatorname{Rad}\left(\beta^{\lambda}\right)$.

As for minimal polarized embeddings for $\Gamma$, we note that in characteristic 3 , the identity matrix $I_{3}=H_{1,2}-H_{2,3}$ satisfies $\operatorname{trace}\left(I_{3} w_{q, m}\right)=0$ for all $(q, m) \in \Gamma$. It follows that in this case the minimal polarized embedding is $\operatorname{PG}(6, \mathbb{F})$. In all other characteristics there is no radical and the minimal polarized embedding is $\operatorname{PG}(7, \mathbb{F})$.

Acknowledgements I would like to thank my friends and colleagues Antonio Pasini and Ilaria Cardinali for their comments on earlier versions of this paper as well as for many discussions on this topic during my visits to the University of Siena. I would also like to thank two anonymous referees for their invaluable comments.

\section{References}

1. Abramenko, P.: Twin Buildings and Applications to S-arithmetic Groups. Lecture Notes in Mathematics, vol. 1641. Springer, Berlin (1996)

2. Adamovich, A.M.: The submodule lattices of Weyl modules of symplectic groups with fundamental highest weights. Vestn. Mosk. Univ. Ser. I Mat. Mekh. (2), 7-10, 112 (1986)

3. Blok, R.J.: On geometries related to buildings. Ph.D. thesis, Delft University of Technology (1999). Supervisor: Prof. Dr. A.E. Brouwer

4. Blok, R.J., Brouwer, A.E.: The geometry far from a residue. In: Groups and Geometries, Siena, 1996. Trends Math., pp. 29-38. Birkhäuser, Basel (1998)

5. Blok, R.J., Brouwer, A.E.: Spanning point-line geometries in buildings of spherical type. J. Geom. 62, 26-35 (1998)

6. Blok, R.J., Pasini, A.: On absolutely universal embeddings. Discrete Math. 267(1-3), 45-62 (2003). Combinatorics 2000 (Gaeta)

7. Bourbaki, N.: Groupes et Algèbres de Lie. Diffusion C.C.L.S., Paris (1975). Chapitres 7 et 8

8. Bourbaki, N.: Groupes et Algèbres de Lie. Masson, Paris (1981). Chapitres 4, 5 et 6

9. Brouwer, A.E.: The complement of a geometric hyperplane in a generalized polygon is usually connected. In: Finite Geometry and Combinatorics, pp. 53-57. Cambridge University Press, Cambridge (1993)

10. Buekenhout, F.: Foundations of incidence geometry. In: Handbook of Incidence Geometry, pp. 63105. North-Holland, Amsterdam (1995)

11. Buekenhout, F., Cohen, A.M.: Diagram Geometry. Book manuscript

12. Buekenhout, F., Lefèvre, C.: Generalized quadrangles in projective spaces. Arch. Math. 25, 540-552 (1974)

13. Cardinali, I., De Bruyn, B., Pasini, A.: Minimal full polarized embeddings of dual polar spaces. J. Algebr. Comb. 25(1), 7-23 (2007)

14. Carter, R.W.: Simple Groups of Lie Type. Pure and Applied Math., vol. 28. Wiley, New York (1972) 
15. Carter, R.W.: Lie Algebras of Finite and Affine Type. Cambridge Studies in Advanced Mathematics, vol. 96. Cambridge University Press, Cambridge (2005)

16. Cohen, A.M.: Point-line spaces related to buildings. In: Buekenhout, F. (ed.) Handbook of Incidence Geometry. North-Holland, Amsterdam (1995). Chapter 12

17. Cooperstein, B.N.: Geometry of groups of Lie type. In: Proceedings of the Conference on Finite Groups, Univ. Utah, Park City, Utah, 1975, pp. 503-512. Academic Press, San Diego (1976)

18. Cooperstein, B.N.: Some geometries associated with parabolic representations of groups of Lie type. Can. J. Math. 28(5), 1021-1031 (1976)

19. Cooperstein, B.N., Shult, E.E.: Geometric hyperplanes of embeddable Lie incidence geometries. In: Hirschfeld, J.W.P., Hughes, D.R., Thas, J.A. (eds.) Advances in Finite Geometries and Designs. Oxford University Press, London (1991). Proceedings of the 3rd Isle of Thorns Conference

20. Cooperstein, B.N., Shult, E.E.: Frames and bases of Lie incidence geometries. J. Geom. 60(1-2), $17-46(1997)$

21. Cooperstein, B.N., Shult, E.E.: Geometric hyperplanes of Lie incidence geometries. Geom. Dedic. 64(1), 17-40 (1997)

22. Dienst, K.J.: Verallgemeinerte Vierecke in pappusschen projektiven Räumen. Geom. Dedic. 9(2), 199-206 (1980)

23. Fulton, W.: Young Tableaux. London Math. Soc. Student Texts, vol. 35. Cambridge University Press, Cambridge (1997)

24. Fulton, W., Harris, J.: Representation Theory. Graduate Texts in Mathematics, vol. 129. Springer, New York (1991). A first course, Readings in Mathematics

25. Humphreys, J.E.: Introduction to Lie Algebras and Representation Theory. Graduate Texts in Math, vol. 9. Springer, New York (1972). Second printing, first edition,

26. Humphreys, J.E.: Finite and infinite dimensional modules for semisimple Lie algebras. In: Lie Theories and Their Applications, Proc. Ann. Sem. Canad. Math. Congr., Queen's Univ., Kingston, Ont., 1977. Queen's Papers in Pure and Appl. Math., vol. 48, pp. 1-64. Queen's Univ., Kingston (1978)

27. Humphreys, J.E.: Modular Representations of Finite Groups of Lie Type. London Mathematical Society Lecture Note Series, vol. 326. Cambridge University Press, Cambridge (2006)

28. Jacobson, N.: Lie Algebras. Interscience Tracts in Pure and Applied Mathematics, vol. 10. Interscience Publishers (a division of John Wiley \& Sons), New York-London (1962)

29. Kasikova, A.: Characterization of some subgraphs of point-collinearity graphs of building geometries. Eur. J. Combin. 28(5), 1493-1529 (2007)

30. Kasikova, A.: Characterization of some subgraphs of point-collinearity graphs of building geometries. II. Adv. Geom. 9(1), 45-84 (2009)

31. Kasikova, A., Shult, E.E.: Absolute embeddings of point-line geometries. J. Algebra 238(1), 265-291 (2001)

32. Lefèvre-Percsy, C.: Polar spaces embedded in a projective space. In: Finite Geometries and Designs. London Math. Soc. Lecture Note Series, vol. 49, pp. 216-220. Cambridge University Press, Cambridge (1981). Proceedings of the 2nd Isle of Thorns Conference

33. Pasini, A.: Diagram Geometries. Oxford Science Publications. Clarendon, Oxford University Press, London (1994)

34. Premet, A.A., Suprunenko, I.D.: The Weyl modules and the irreducible representations of the symplectic group with the fundamental highest weights. Commun. Algebra 11(12), 1309-1342 (1983)

35. Ree, R.: On some simple groups defined by C. Chevalley. Trans. Am. Math. Soc. 84, 392-400 (1957)

36. Ronan, M.A.: Embeddings and hyperplanes of discrete geometries. Eur. J. Combin. 8, 179-185 (1987)

37. Ronan, M.A.: Lectures on Buildings. Perspectives in Mathematics, vol. 7. Academic Press, San Diego (1989)

38. Shult, E.E.: Geometric hyperplanes of embeddable Grassmannians. J. Algebra 145, 55-82 (1992)

39. Shult, E.E.: Geometric hyperplanes of the half-spin geometries arise from embeddings. Bull. Bel. Math. Soc. 1(3), 439-453 (1994). Dedicated to J.A. Thas on his fiftieth birthday

40. Shult, E.E.: Embeddings and hyperplanes of Lie incidence geometries. In: Groups of Lie Type and Their Geometries, Como, 1993. London Math. Soc. Lecture Note Ser., vol. 207, pp. 215-232. Cambridge University Press, Cambridge (1995)

41. Shult, E.E.: Embeddings and hyperplanes of the lie incidence geometry of type $e_{7,1}$. J. Geom. 59, 152-172 (1997)

42. Shult, E.E.: Points and Lines. Book manuscript

43. Shult, E.E., Thas, J.A.: Hyperplanes of dual polar spaces and the spin module. Arch. Math. 59, 610-23 (1992) 
44. Steinberg, R.: Representations of algebraic groups. Nagoya Math. J. 22, 33-56 (1963)

45. Steinberg, R.: Lectures on Chevalley Groups. Yale Lecture Notes. Yale University Press, New Haven (1967)

46. Steinberg, R.: Endomorphisms of Linear Algebraic Groups. Memoirs of the American Mathematical Society, vol. 80. Am. Math. Soc., Providence (1968)

47. Thas, J.A., Van Maldeghem, H.: On embeddings of the flag geometries of projective planes in finite projective spaces. Designs Codes Cryptogr. 17(1-3), 97-104 (1999)

48. Thas, J.A., Van Maldeghem, H.: Embeddings of small generalized polygons. Finite Fields Appl. 12(4), 565-594 (2006)

49. Tits, J.: A local approach to buildings. In: The Geometric Vein, pp. 519-547. Springer, New York (1981)

50. Tits, J.: Buildings and group amalgamations. In: Proceedings of Groups-St. Andrews 1985. London Math. Soc. Lecture Note Ser., vol. 121, pp. 110-127. Cambridge University Press, Cambridge (1986)

51. Veblen, O., Young, J.W. (eds.): Projective Geometry. Ginn and Company, Boston (1910)

52. Verma, D.: The rôle of affine Weyl groups in the representation theory of algebraic Chevalley groups and their Lie algebras. In: Lie Groups and Their Representations, Proc. Summer School, Bolyai János Math. Soc., Budapest, 1971, pp. 653-705. Halsted, New York (1975)

53. Weiss, R.M.: The Structure of Spherical Buildings. Princeton University Press, Princeton (2003)

54. Wong, W.J.: Representations of Chevalley groups in characteristic p. Nagoya Math. J. 45, 39-78 (1972) 